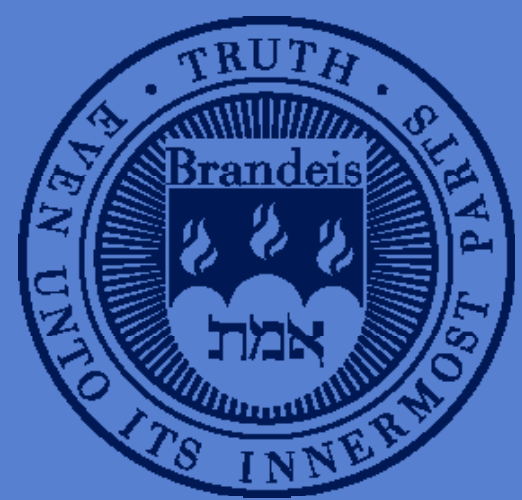

\title{
Inference on Causal Effects in a Generalized Regression Kink Design
}

Zhuan Pei, Brandeis University, David Card, UC Berkeley, NBER and IZA, David S. Lee, Princeton University and NBER, Andrea Weber, University of Mannheim and IZA

\section{Working Paper Series}




\title{
Inference on Causal Effects in a Generalized Regression Kink Design ${ }^{1}$
}

\author{
David Card \\ UC Berkeley, NBER and IZA
}

David S. Lee

Princeton University and NBER

\author{
Zhuan Pei \\ Brandeis University
}

\author{
Andrea Weber \\ University of Mannheim and IZA
}

January 162015

\begin{abstract}
We consider nonparametric identification and estimation in a nonseparable model where a continuous regressor of interest is a known, deterministic, but kinked function of an observed assignment variable. This design arises in many institutional settings where a policy variable (such as weekly unemployment benefits) is determined by an observed but potentially endogenous assignment variable (like previous earnings). We provide new results on identification and estimation for these settings, and apply our results to obtain estimates of the elasticity of joblessness with respect to UI benefit rates. We characterize a broad class of models in which a sharp "Regression Kink Design" (RKD, or RK Design) identifies a readily interpretable treatment-on-the-treated parameter (Florens et al. (2008)). We also introduce a "fuzzy regression kink design" generalization that allows for omitted variables in the assignment rule, noncompliance, and certain types of measurement errors in the observed values of the assignment variable and the policy variable. Our identifying assumptions give rise to testable restrictions on the distributions of the assignment variable and predetermined covariates around the kink point, similar to the restrictions delivered by Lee (2008) for the regression discontinuity design. We then use a fuzzy RKD approach to study the effect of unemployment insurance benefits on the duration of joblessness in Austria, where the benefit schedule has kinks at the minimum and maximum benefit level. Our preferred estimates suggest that changes in UI benefit generosity exert a relatively large effect on the duration of joblessness of both low-wage and high-wage UI recipients in Austria.
\end{abstract}

JEL codes: C13, C14, C31

Keywords: Regression Discontinuity Design; Regression Kink Design; Treatment Effects; Nonseparable Models; Nonparametric Estimation

\footnotetext{
${ }^{1}$ We thank Diane Alexander, Mingyu Chen, Kwabena Donkor, Martina Fink, Samsun Knight, Andrew Langan, Carl Lieberman, Michelle Liu, Steve Mello, Rosa Weber and Pauline Leung for excellent research assistance. We have benefited from the comments and suggestions of Sebastian Calonico, Matias Cattaneo, Andrew Chesher, Nathan Grawe, Bo Honoré, Guido Imbens, Pat Kline and seminar participants at Brandeis, BYU, Brookings, Cornell, Georgetown, GWU, IZA, LSE, Michigan, NAESM, NBER, Princeton, Rutgers, SOLE, Upjohn, UC Berkeley, UCL, Uppsala, Western Michigan, Wharton and Zürich. Andrea Weber gratefully acknowledges research funding from the Austrian Science Fund (NRN Labor Economics and the Welfare State).
} 


\section{Introduction}

A growing body of research considers the identification and estimation of nonseparable models with continuous endogenous regressors in semiparametric (e.g., Lewbel (1998); Lewbel (2000)) and non-parametric settings (e.g., Blundell and Powell (2003); Chesher (2003); Florens et al. (2008); Imbens and Newey (2009)). The methods proposed in the literature so far rely on instrumental variables that are independent of the unobservable terms in the model. Unfortunately, independent instruments are often hard to find, particularly when the regressor of interest is a deterministic function of an endogenous assignment variable. Unemployment benefits, for example, are set as function of previous earnings in most countries. Any variable that is correlated with benefits is likely to be correlated with the unobserved determinants of previous wages and is therefore unlikely to satisfy the necessary independence assumptions for a valid instrument.

Nevertheless, many tax and benefit formulas are piece-wise linear functions with kinks in the relationship between the assignment variable and the policy variable caused by minimums, maximums, and discrete shifts in the marginal tax or benefit rate. As noted by Classen (1977), Welch (1977), Guryan (2001), Dahlberg et al. (2008), Nielsen et al. (2010) and Simonsen et al. (Forthcoming), a kinked assignment rule holds out the possibility for identification of the policy variable effect even in the absence of traditional instruments. The idea is to look for an induced kink in the mapping between the assignment variable and the outcome variable that coincides with the kink in the policy rule, and compare the relative magnitudes of the two kinks.

This paper establishes conditions under which the behavioral response to a formulaic policy variable like unemployment benefits can be identified within a general class of nonparametric and nonseparable regression models. Specifically, we establish conditions for the regression kink design (RKD) to identify the "local average response" defined by Altonji and Matzkin (2005) or the "treatment-on-the-treated" parameter defined by Florens et al. (2008). The key assumption is that conditional on the unobservable determinants of the outcome variable, the density of the assignment variable is smooth (i.e., continuously differentiable) at the kink point in the policy rule. We show that this smooth density condition rules out deterministic sorting while allowing less extreme forms of endogeneity - including, for example, situations where agents endogenously sort but make small optimization errors (e.g., Chetty (2012)). We also show that the smooth density condition generates testable predictions for the distribution of predetermined covariates among the population of agents located near the kink point. Thus, as in a regression discontinuity (RD) design (Lee 
and Lemieux (2010); DiNardo and Lee (2011)), the validity of the regression kink design can be evaluated empirically.

In many realistic settings, the policy rule of interest depends on unobserved individual characteristics, or is implemented with error. In addition, both the assignment variable and the policy variable may be observed with error. We present a generalization of the RKD - which we call a "fuzzy regression kink design" - that allows for these features. The fuzzy RKD estimand replaces the known change in slope of the assignment rule at the kink with an estimate based on the observed data. Under a series of additional assumptions, including a monotonicity condition analogous to the one introduced by Imbens and Angrist (1994) (and implicit in latent index models (Vytlacil, 2002)), we show that the fuzzy RKD identifies a weighted average of marginal effects, where the weights are proportional to the magnitude of the individual-specific kinks. ${ }^{1}$

We then review and extend existing methods for the nonparametric estimation of RKD using local polynomial estimation, including Fan and Gijbels (1996) - hereafter, FG; Imbens and Kalyanaraman (2012) hereafter IK; and Calonico et al. (Forthcoming) - hereafter, CCT. And finally, we use a fuzzy RKD approach to analyze the effect of unemployment insurance (UI) benefits on the duration of joblessness in Austria. As in the United States, the Austrian UI system specifies a benefit level that is proportional to earnings in a base period prior to job loss, subject to a minimum and maximum. We study the effects of the kinks at the minimum and maximum benefit levels, using data on a large sample of jobless spells from the Austrian Social Security Database (see Zweimüller et al. (2009)). Simple plots of the data show relatively strong visual evidence of kinks in the relationship between base period earnings and the durations of joblessness at both kink points. We also examine the relationship between base period earnings and various predetermined covariates (such as gender, age, and occupation) around the kink points, checking whether the conditional distributions of the covariates evolve smoothly around the kink points.

We present a range of alternative estimates of the behavioral effect of higher benefits on the duration of joblessness derived from local linear and local quadratic polynomial models using various bandwidth selection algorithms (including FG, IK, and CCT, and extensions of IK and CCT for the fuzzy RKD case). For each of the alternative choices of polynomial order and bandwidth selector we show conventional kink estimates and corresponding "bias-corrected" estimates that incorporate the correction suggested by Calonico et al. (Forthcoming). We also investigate the empirical performance of the alternative estimators using simula-

\footnotetext{
${ }^{1}$ The marginal effects of interest in this paper refer to derivatives of an outcome variable with respect to a continuous endogenous regressor, and should not be confused with the marginal treatment effects defined in Heckman and Vytlacil (2005), where the treatment is binary.
} 
tion studies of data generating processes (DGP's) that are closely based on our actual data. In our empirical setting, we find that local quadratic estimators have substantially larger (asymptotic) mean squared errors than local linear estimators and that CCT's bias correction procedure leads to a loss in precision with only a modest offsetting reduction in bias. Our preferred estimates - derived from uncorrected local linear models using the FG bandwidth selection procedure- imply that changes in UI benefit generosity exert a relatively large effect on the duration of joblessness of UI recipients in Austria.

\section{Nonparametric Regression and the Regression Kink Design}

\subsection{Background}

Consider the generalized nonseparable model

$$
Y=y(B, V, U)
$$

where $Y$ is an outcome, $B$ is a continuous regressor of interest, $V$ is another observed covariate, and $U$ is a potentially multi-dimensional error term that enters the function $y$ in a non-additive way. This is a particular case of the model considered by Imbens and Newey (2009); there are two observable covariates and interest centers on the effect of $B$ on $Y$. As noted by Imbens and Newey (2009), this setup is general enough to encompass a variety of treatment effect models. When $B$ is binary, the treatment effect for a particular individual is given by $Y_{1}-Y_{0}=y(1, V, U)-y(0, V, U)$; when $B$ is continuous, the treatment effect is $\frac{\partial}{\partial b} Y=\frac{\partial}{\partial b} y(b, V, U)$. In settings with discrete outcomes, $Y$ could be defined as an individual-specific probability of a particular outcome (as in a binary response model) or as an individual-specific expected value (e.g. an expected duration) that depends on $B, V$, and $U$, where the structural function of interest is the relation between $B$ and the probability or expected value. ${ }^{2}$

For the continuous regressor case, Florens et al. (2008) define the "treatment on the treated" (TT) as

$$
T T_{b \mid v}(b, v)=\int \frac{\partial y(b, v, u)}{\partial b} d F_{U \mid B=b, V=v}(u)
$$

where $F_{U \mid B=b, V=v}(u)$ is the c.d.f. of $U$ conditional on $B=b, V=v$. As noted by Florens et al. (2008), this

\footnotetext{
${ }^{2}$ In these cases, one would use the observed outcome $Y^{O}$ (a discrete outcome, or an observed duration), and use the fact that the expectation of $Y^{O}$ and $Y$ are equivalent given the same conditioning statement, in applying all of the identification results below.
} 
is equivalent to the "local average response" (LAR) parameter of Altonji and Matzkin (2005). The TT (or equivalently the LAR) gives the average effect of a marginal increase in $b$ at some specific value of the pair $(b, v)$, holding fixed the distribution of the unobservables, $F_{U \mid B=b, V=v}(\cdot)$.

Recent studies, including Florens et al. (2008) and Imbens and Newey (2009), have proposed methods that use an instrumental variable $Z$ to identify causal parameters such as TT or LAR. An appropriate instrument $Z$ is assumed to influence $B$, but is also assumed to be independent of the non-additive errors in the model. Chesher (2003) observes that such independence assumptions may be "strong and unpalatable", and hence proposes the use of local independence of $Z$ to identify local effects.

As noted in the introduction, there are some important contexts where no instruments can plausibly satisfy the independence assumption, either globally or locally. For example, consider the case where $Y$ represents the expected duration of unemployment for a job loser, $B$ represents the level of unemployment benefits, and $V$ represents pre-job-loss earnings. Assume (as in many institutional settings) that unemployment benefits are a linear function of pre-job-loss earnings up to some maximum: i.e., $B=b(V)=\rho \min (V, T)$. Conditional on $V$, there is no variation in the benefit level, so model (1) is not nonparametrically identified. One could try to get around this fundamental non-identification by treating $V$ as an error component correlated with $B$. But in this case, any variable that is independent of $V$ will, by construction, be independent of the regressor of interest $B$, so it will not be possible to find instruments for $B$, holding constant the policy regime.

Nevertheless, it may be possible to exploit the kink in the benefit rule to identify the causal effect of $B$ on $Y$. The idea is that if $B$ exerts a causal effect on $Y$, and there is a kink in the deterministic relation between $B$ and $V$ at $v=T$, then we should expect to see an induced kink in the relationship between $Y$ and $V$ at $v=T{ }^{3}$ Using the kink for identification is in a similar spirit to the regression discontinuity design of Thistlethwaite and Campbell (1960), but the RD approach cannot be directly applied when the benefit formula $b()$ is continuous. This kink-based identification strategy has been employed in a few empirical studies. Guryan (2001), for example, uses kinks in state education aid formulas as part of an instrumental variables strategy to study the effect of public school spending. ${ }^{4}$ Dahlberg et al. (2008) use the same approach to estimate the

\footnotetext{
${ }^{3}$ Without loss of generality, we normalize the kink threshold $T$ to 0 in the remainder of our theoretical presentation.

${ }^{4}$ Guryan (2001) describes the identification strategy as follows: "In the case of the Overburden Aid formula, the regression includes controls for the valuation ratio, 1989 per-capita income, and the difference between the gross standard and 1993 education expenditures (the standard of effort gap). Because these are the only variables on which Overburden Aid is based, the exclusion restriction only requires that the functional form of the direct relationship between test scores and any of these variables is not the same as the functional form in the Overburden Aid formula."
} 
impact of intergovernmental grants on local spending and taxes. More recently, Simonsen et al. (Forthcoming) use a kinked relationship between total expenditure on prescription drugs and their marginal price to study the price sensitivity of demand for prescription drugs. Nielsen et al. (2010), who introduce the term "Regression Kink Design" for this approach, use a kinked student aid scheme to identify the effect of direct costs on college enrollment.

Nielsen et al. (2010) make precise the assumptions needed to identify the causal effects in the constanteffect, additive model

$$
Y=\tau B+g(V)+\varepsilon,
$$

where $B=b(V)$ is assumed to be a deterministic (and continuous) function of $V$ with a kink at $V=0$. They show that if $g(\cdot)$ and $E[\varepsilon \mid V=v]$ have derivatives that are continuous in $v$ at $v=0$, then

$$
\tau=\frac{\left.\lim _{v_{0} \rightarrow 0^{+}} \frac{d E[Y \mid V=v]}{d v}\right|_{v=v_{0}}-\left.\lim _{v_{0} \rightarrow 0^{-}} \frac{d E[Y \mid V=v]}{d v}\right|_{v=v_{0}}}{\lim _{v_{0} \rightarrow 0^{+}} b^{\prime}\left(v_{0}\right)-\lim _{v_{0} \rightarrow 0^{-}} b^{\prime}\left(v_{0}\right)} .
$$

The expression on the right hand side of this equation - the RKD estimand - is simply the change in slope of the conditional expectation function $E[Y \mid V=v]$ at the kink point $(v=0)$, divided by the change in the slope of the deterministic assignment function $b(\cdot)$ at $0 .{ }^{5}$

Also related are papers by Dong and Lewbel (2014) and Dong (2013), which derive identification results using kinks in a regression discontinuity setting. Dong and Lewbel (2014) show that the derivative of the RD treatment effect with respect to the running variable, which the authors call TED, is nonparametrically identified. Under a local policy invariance assumption, TED can be interpreted as the change in the treatment effect that would result from a marginal change in the RD threshold. More closely related to our study is Dong (2013), which shows that identification in an RD design can be achieved in the absence of a first stage discontinuity, provided there is a kink in the treatment probability at the RD cutoff. In Remark 6 below, we provide an example where such a kink could be expected. Dong (2013) also shows that a slope and level change in the treatment probability can both be used to identify the RD treatment effect with a local constant treatment effect restriction; we discuss an analogous point in the RK design in Remark 3.

Below we provide the following new identification results. First, we establish identification conditions for the RK design in the context of the general nonseparable model (1). By allowing the error term to

\footnotetext{
${ }^{5}$ In an earlier working paper version, Nielsen et al. (2010) provide similar conditions for identification for a less restrictive, additive model, $Y=g(B, V)+\varepsilon$.
} 
enter nonseparably, we are allowing for unrestricted heterogeneity in the structural relation between the endogenous regressor and the outcome. As an example of the relevance of this generalization, consider the case of modeling the impact of UI benefits on unemployment durations with a proportional hazards model. Even if UI benefits enter the hazard function with a constant coefficient, the shape of the baseline hazard will in general cause the true model for expected durations to be incompatible with the constant-effects, additive specification in (2). The addition of multiplicative unobservable heterogeneity (as in Meyer (1990)) to the baseline hazard poses an even greater challenge to the justification of parametric specifications such as (2). The nonseparable model (1), however, contains the implied model for durations in Meyer (1990) as a special case, and goes further by allowing (among other things) the unobserved heterogeneity to be correlated with $V$ and $B$. Having introduced unobserved heterogeneity in the structural relation, we show that the RKD estimand $\tau$ identifies an effect that can be viewed as the TT (or LAR) parameter. Given that the identified effect is an average of marginal effects across a heterogeneous population, we also make precise how the RKD estimand implicitly weights these heterogeneous marginal effects. The weights are intuitive and correspond to the weights that would determine the slope of the experimental response function in a randomized experiment.

Second, we generalize the RK design to allow for the presence of unobserved determinants of $B$ and measurement errors in $B$ and $V$. That is, while maintaining the model in (1), we allow for the possibility that the observed value for $B$ deviates from the amount predicted by the formula using $V$, either because of unobserved inputs in the formula, noncompliance behavior or measurement errors in $V$ or $B$. This "fuzzy RKD" generalization may have broader applicability than the "sharp RKD". 6

Finally, we provide testable implications for a valid RK design. As we discuss below, a key condition for identification in the RKD is that the distribution of $V$ for each individual is sufficiently smooth. This smooth density condition rules out the case where an individual can precisely manipulate $V$, but allows individuals to exert some influence over $V .{ }^{7}$ We provide two tests that can be useful in assessing whether this key identifying assumption holds in practice.

\footnotetext{
${ }^{6}$ The sharp/fuzzy distinction in the RKD is analogous to that for the RD Design (see Hahn et al. (2001)).

${ }^{7}$ Lee (2008) requires a similar identifying condition in a regression discontinuity design. Even though the smooth density condition is not necessary for an RD design, it leads to many intuitive testable implications, which the minimal continuity assumptions in Hahn et al. (2001) do not.
} 


\subsection{Identification of Regression Kink Designs}

\subsubsection{Sharp RKD}

We begin by stating the identifying assumptions for the RKD and making precise the interpretation of the resulting causal effect. In particular, we provide conditions under which the RKD identifies the $T T_{b \mid v}$ parameter defined above.

Sharp RK Design: Let $(V, U)$ be a pair of random variables (with $V$ observable and $U$ unobservable). While the running variable $V$ is one-dimensional, the error term $U$ need not be, and this unrestricted dimensionality of heterogeneity makes the nonseparable model (1) equivalent to treatment effects models as mentioned in subsection 2.1. Denote the c.d.f. and p.d.f. of $V$ conditional on $U=u$ by $F_{V \mid U=u}(v)$ and $f_{V \mid U=u}(v)$. Define $B \equiv b(V), Y \equiv y(B, V, U), y_{1}(b, v, u) \equiv \frac{\partial y(b, v, u)}{\partial b}$ and $y_{2}(b, v, u) \equiv \frac{\partial y(b, v, u)}{\partial v}$. Let $I_{V}$ be an arbitrarily small closed interval around the cutoff 0 and $I_{b(V)} \equiv\left\{b \mid b=b(v)\right.$ for some $\left.v \in I_{V}\right\}$ be the image of $I_{V}$ under the mapping $b$. In the remainder of this section, we use the notation $I_{S_{1}, \ldots, S_{k}}$ to denote the product space $I_{S_{1}} \times \ldots \times I_{S_{k}}$ where the $S_{j}$ 's are random variables.

Assumption 1. (Regularity) (i) The support of $U$ is bounded: it is a subset of the arbitrarily large compact set $I_{U} \subset \mathbb{R}^{m}$. (ii) $y(\cdot, \cdot, \cdot)$ is a continuous function and is partially differentiable w.r.t. its first and second arguments. In addition, $y_{1}(b, v, u)$ is continuous on $I_{b(V), V, U}$.

Assumption 2. (Smooth effect of $V) y_{2}(b, v, u)$ is continuous on $I_{b(V), V, U}$.

Assumption 3. (First stage and non-negligible population at the kink) (i) $b(\cdot)$ is a known function, everywhere continuous and continuously differentiable on $I_{V} \backslash\{0\}$, but $\lim _{v \rightarrow 0^{+}} b^{\prime}(v) \neq \lim _{v \rightarrow 0^{-}} b^{\prime}(v)$. (ii) The set $A_{U}=\left\{u: f_{V \mid U=u}(v)>0 \forall v \in I_{V}\right\}$ has a positive measure under $U: \int_{A_{U}} d F_{U}(u)>0$.

Assumption 4. (Smooth density) The conditional density $f_{V \mid U=u}(v)$ and its partial derivative w.r.t. $v$, $\frac{\partial f_{V \mid U=u}(v)}{\partial v}$, are continuous on $I_{V, U}$.

Assumption 1(i) can be relaxed, but other regularity conditions, such as the dominance of $y_{1}$ by an integrable function with respect to $F_{U}$, will be needed instead to allow for the interchange of differentiation and integration in proving Proposition 1 below. Assumption 1(ii) states that the marginal effect of $B$ must be a continuous function of the observables and the unobserved error $U$. Assumption 2 is considerably weaker than an exclusion restriction that dictates $V$ not enter as an argument, because here $V$ is allowed to affect $Y$, as long as its marginal effect is continuous. In the context of UI, for example, pre-job-loss earnings may independently affect unemployment duration, but Assumption 2 is satisfied as long as the 
relationship between pre-job-loss earnings is smooth across the threshold. Assumption 3(i) states that the researcher knows the function $b(v)$, and that there is a kink in the relationship between $B$ and $V$ at the threshold $V=0$. The continuity of $b(v)$ may appear restrictive, as it rules out the case where the level of $b(v)$ also changes at $v=0$, but its necessity stems from the flexibility of our model, which we discuss in more detail in Remark 3. Assumption 3(ii) states that the density of $V$ must be positive around the threshold for a nontrivial subpopulation.

Assumption 4 is the key identifying assumption for a valid RK design. But whereas continuity of $f_{V \mid U=u}(v)$ in $v$ is sufficient for identification in the RD design, it is insufficient in the RK design. Instead, the sufficient condition is the continuity of the partial derivative of $f_{V \mid U=u}(v)$ with respect to $v$. In subsection 4.1 below we discuss a simple equilibrium search model where Assumption 4 may or may not hold. The importance of this assumption underscores the need to be able to empirically test its implications.

Proposition 1. In a valid Sharp RKD, that is, when Assumptions 1-4 hold:

(a) $\operatorname{Pr}(U \leqslant u \mid V=v)$ is continuously differentiable in $v$ at $v=0 \forall u \in I_{U}$.

(b) $\frac{\left.\lim _{v_{0} \rightarrow 0^{+}} \frac{d E[Y \mid V=v]}{d v}\right|_{v=v_{0}}-\left.\lim _{v_{0} \rightarrow 0^{-}} \frac{d E[Y \mid V=v]}{d v}\right|_{v=v_{0}}}{\left.\lim _{v_{0} \rightarrow 0^{+}} \frac{d b(v)}{d v}\right|_{v=v_{0}}-\left.\lim _{v_{0} \rightarrow 0^{-}} \frac{d b(v)}{d v}\right|_{v=v_{0}}}=E\left[y_{1}\left(b_{0}, 0, U\right) \mid V=0\right]=\int_{u} y_{1}\left(b_{0}, 0, u\right) \frac{f_{V \mid U=u}(0)}{f_{V}(0)} d F_{U}(u)=T T_{b_{0} \mid 0}$ where $b_{0}=b(0)$.

Proof: For part (a), we apply Bayes' Rule and write

$$
\operatorname{Pr}(U \leqslant u \mid V=v)=\int_{A} \frac{f_{V \mid U=u^{\prime}}(v)}{f_{V}(v)} d F_{U}\left(u^{\prime}\right) .
$$

where $A=\left\{u^{\prime}: u^{\prime} \leqslant u\right\}$. The continuous differentiability of $\operatorname{Pr}(U \leqslant u \mid V=v)$ in $v$ follows from Lemma 1 and Lemma 2 in subsection A.1 of the Supplemental Appendix.

For part (b), in the numerator

$$
\begin{aligned}
\left.\lim _{v_{0} \rightarrow 0^{+}} \frac{d E[Y \mid V=v]}{d v}\right|_{v=v_{0}}= & \left.\lim _{v_{0} \rightarrow 0^{+}} \frac{d}{d v} \int y(b(v), v, u) \frac{f_{V \mid U=u}(v)}{f_{V}(v)} d F_{U}(u)\right|_{v=v_{0}} \\
= & \left.\lim _{v_{0} \rightarrow 0^{+}} \int \frac{\partial}{\partial v} y(b(v), v, u) \frac{f_{V \mid U=u}(v)}{f_{V}(v)} d F_{U}(u)\right|_{v=v_{0}} \\
= & \lim _{v_{0} \rightarrow 0^{+}} b^{\prime}\left(v_{0}\right) \int y_{1}\left(b\left(v_{0}\right), v_{0}, u\right) \frac{f_{V \mid U=u}\left(v_{0}\right)}{f_{V}\left(v_{0}\right)} d F_{U}(u)+ \\
& \lim _{v_{0} \rightarrow 0^{+}} \int\left\{y_{2}\left(b\left(v_{0}\right), v_{0}, u\right) \frac{f_{V \mid U=u}\left(v_{0}\right)}{f_{V}\left(v_{0}\right)}+y\left(b\left(v_{0}\right), v_{0}, u\right) \frac{\partial}{\partial v} \frac{f_{V \mid U=u}\left(v_{0}\right)}{f_{V}\left(v_{0}\right)}\right\} d F_{U}(u) .
\end{aligned}
$$

A similar expression is obtained for $\left.\lim _{v_{0} \rightarrow 0^{-}} \frac{d E[Y \mid V=v]}{d v}\right|_{v=v_{0}}$. The bounded support and continuity in Assumptions 1-4 allow differentiating under the integral sign per Roussas (2004) (p. 97). We also invoke the 
dominated convergence theorem allowed by the continuity conditions over a compact set in order to exchange the limit operator and the integral. It implies that the difference in slopes above and below the kink threshold can be simplified to:

$$
\begin{aligned}
& \left.\lim _{v_{0} \rightarrow 0^{+}} \frac{d E[Y \mid V=v]}{d v}\right|_{v=v_{0}}-\left.\lim _{v_{0} \rightarrow 0^{-}} \frac{d E[Y \mid V=v]}{d v}\right|_{v=v_{0}} \\
= & \left(\lim _{v \rightarrow 0^{+}} b^{\prime}\left(v_{0}\right)-\lim _{v \rightarrow 0^{-}} b^{\prime}\left(v_{0}\right)\right) \int y_{1}(b(0), 0, u) \frac{f_{V \mid U=u}(0)}{f_{V}(0)} d F_{U}(u) .
\end{aligned}
$$

Assumption 3(i) states that the denominator $\lim _{v_{0} \rightarrow 0^{+}} b^{\prime}\left(v_{0}\right)-\lim _{v_{0} \rightarrow 0^{-}} b^{\prime}\left(v_{0}\right)$ is nonzero, and hence we have

$$
\frac{\left.\lim _{v_{0} \rightarrow 0^{+}} \frac{d E[Y \mid V=v]}{d v}\right|_{v=v_{0}}-\left.\lim _{v_{0} \rightarrow 0^{-}} \frac{d E[Y \mid V=v]}{d v}\right|_{v=v_{0}}}{\lim _{v_{0} \rightarrow 0^{+}} b^{\prime}\left(v_{0}\right)-\lim _{v_{0} \rightarrow 0^{-}} b^{\prime}\left(v_{0}\right)}=E\left[y_{1}(b(0), 0, U) \mid V=0\right]=\int y_{1}(b(0), 0, u) \frac{f_{V \mid U=u}(0)}{f_{V}(0)} d F_{U}(u),
$$

which completes the proof.

Part (a) states that the rate of change in the probability distribution of individual types with respect to the assignment variable $V$ is continuous at $V=0 .{ }^{8}$ This leads directly to part (b): as a consequence of the smoothness in the underlying distribution of types around the kink, the discontinuous change in the slope of $E[Y \mid V=v]$ at $v=0$ divided by the discontinuous change in slope in $b(V)$ at the kink point identifies $T T_{b_{0} \mid 0 \cdot} \cdot$

Remark 1. It is tempting to interpret $T T_{b_{0} \mid 0}$ as the "average marginal effect of $B$ for individuals with $V=0$ ", which may seem very restrictive because the smooth density condition implies that $V=0$ is a measure-zero event. However, part (b) implies that $T T_{b_{0} \mid 0}$ is a weighted average of marginal effects across the entire population, where the weight assigned to an individual of type $U$ reflects the relative likelihood that he or she has $V=0$. In settings where $U$ is highly correlated with $V, T T_{b_{0} \mid 0}$ is only representative of the treatment effect for agents with realizations of $U$ that are associated with values of $V$ close to 0 . In settings where $V$ and $U$ are independent, the weights for different individuals are equal, and RKD identifies the average marginal effect evaluated at $B=b_{0}$ and $V=0$.

\footnotetext{
${ }^{8}$ Note also that Proposition 1(a) implies Proposition 2(a) in Lee (2008), i.e., the continuity of $\operatorname{Pr}(U \leqslant u \mid V=v)$ at $v=0$ for all $u$. This is a consequence of the stronger smoothness assumption we have imposed on the conditional distribution of $V$ on $U$.

${ }^{9}$ Technically, the $T T$ and $L A R$ parameters do not condition on a second variable $V$. But in the case where there is a one-toone relationship between $B$ and $V$, the trivial integration over the (degenerate) distribution of $V$ conditional on $B=b_{0}$ will imply that $T T_{b_{0} \mid 0}=T T_{b_{0}} \equiv E\left[y_{1}\left(b_{0}, V, U\right) \mid B=b_{0}\right]$, which is literally the $T T$ parameter discussed in Florens et al. (2008) and the $L A R$ discussed in Altonji and Matzkin (2005). In our application to unemployment benefits, $B$ and $V$ are not one-to-one, since beyond $V=0, B$ is at the maximum benefit level. In this case, $T T_{b}$ will in general be discontinuous with respect to $b$ at $b_{0}$ :

$$
T T_{b}= \begin{cases}T T_{b \mid v} & b<b_{0} \\ \int T T_{b_{0} \mid v} f_{V \mid B}\left(v \mid b_{0}\right) d v & b=b_{0}\end{cases}
$$

and the RKD estimand identifies $\lim _{b \uparrow b_{0}} T T_{b}$.
} 
Remark 2. The weights in Proposition 1 are the same ones that would be obtained from using a randomized experiment to identify the average marginal effect of $B$, evaluated at $B=b_{0}, V=0$. That is, suppose that $B$ was assigned randomly so that $f_{B \mid V, U}(b)=f(b)$. In such an experiment, the identification of an average marginal effect of $b$ at $V=0$ would involve taking the derivative of the experimental response surface $E[Y \mid B=b, V=v]$ with respect to $b$ for units with $V=0$. This would yield

$$
\begin{aligned}
\left.\frac{\partial E[Y \mid B=b, V=0]}{\partial b}\right|_{b=b_{0}} & =\left.\frac{\partial\left(\int y(b, 0, u) d F_{U \mid V=0, B=b}(u)\right)}{\partial b}\right|_{b=b_{0}} \\
& =\left.\frac{\partial\left(\int_{u} y(b, 0, u) \frac{f_{B \mid V=0, U=u}(b)}{f_{B \mid V=0}(b)} \frac{f_{V \mid U=u}(0)}{\partial b} d F_{U}(u)\right)}{f_{V}(0)}\right|_{b=b_{0}} \\
& =\left.\frac{\partial\left(\int y(b, 0, u) \frac{f_{V \mid U=u}(0)}{f_{V}(0)} d F_{U}(u)\right)}{\partial b}\right|_{b=b_{0}} \\
& =\int y_{1}\left(b_{0}, 0, u\right) \frac{f_{V \mid U=u}(0)}{f_{V}(0)} d F_{U}(u) .
\end{aligned}
$$

Even though $B$ is randomized in this hypothetical experiment, $V$ is not. Intuitively, although randomization allows one to identify marginal effects of $B$, it cannot resolve the fact that units with $V=0$ will in general have a particular distribution of $U$. Of course, the advantage of this hypothetical randomized experiment is that one could potentially identify the average marginal effect of $B$ at all values of $B$ and $V$, and not just at $B=b_{0}$ and $V=0$.

Remark 3. In the proof of Proposition 1, we need the continuity of $b(v)$ to ensure that the left and right limits of $y_{1}\left(b\left(v_{0}\right), v_{0}, u\right), y_{2}\left(b\left(v_{0}\right), v_{0}, u\right)$ and $y\left(b\left(v_{0}\right), v_{0}, u\right)$ are the same as $v_{0}$ approaches 0 . In the case where both the slope and the level of $b(v)$ change at $v=0$, the RK estimand does not point identify an interpretable treatment effect in the nonseparable model (1). The RD estimand, however, still identifies an average treatment effect. In subsection A.2 of the Supplemental Appendix, we show:

$$
\frac{\lim _{v_{0} \rightarrow 0^{+}} E\left[Y \mid V=v_{0}\right]-\lim _{v_{0} \rightarrow 0^{-}} E\left[Y \mid V=v_{0}\right]}{\lim _{v_{0} \rightarrow 0^{+}} b\left(v_{0}\right)-\lim _{v_{0} \rightarrow 0^{-}} b\left(v_{0}\right)}=E\left[y_{1}(\tilde{b}, 0, U) \mid V=0\right]
$$

where $\tilde{b}$ is a value between $\lim _{v_{0} \rightarrow 0^{-}} b\left(v_{0}\right)$ and $\lim _{v_{0} \rightarrow 0^{+}} b\left(v_{0}\right)$. In the special case of a constant treatment effect model like (2), the RD and RK design both identify the same causal effect parameter. In the absence of strong a priori knowledge about treatment effect homogeneity, however, it seems advisable to use an RD design. ${ }^{10}$

\footnotetext{
${ }^{10}$ Turner (2013) studies the effect of the Pell Grant program in the United States. The formula for these grants has both a discontinuity and a slope change at the grant eligibility threshold. She argues that the status of being a Pell Grant recipient, $D$, may impact $Y$ independently from the marginal financial effect of $B$ on $Y$ (i.e., $Y=y(B, D, V, U)$ ), and she studies the identification of
} 


\subsubsection{Fuzzy Regression Kink Design}

Although many important policy variables are set according to a deterministic formula, in practice there is often some slippage between the theoretical value of the variable as computed by the stated rule and its observed value. This can arise when the formula - while deterministic - depends on other (unknown) variables in addition to the primary assignment variable, when there is non-compliance with the policy formula, or when measurement errors are present in the available data set. This motivates the extension to a fuzzy RKD. ${ }^{11}$

Specifically, assume now that $B=b(V, \varepsilon)$, where the presence of $\varepsilon$ in the formula for $B$ allows for unobserved determinants of the policy formula and non-compliant behavior. The vector $\varepsilon$ is potentially correlated with $U$ and therefore also with the outcome variable $Y$. As an illustration, consider the simple case where the UI benefit formula depends on whether or not a claimant has dependents. Let $\mathrm{D}$ be a claimant with dependents and let $\mathrm{N}$ be a claimant with no dependents, and let $b^{1}(v)$ and $b^{0}(v)$ be the benefit formulas for $\mathrm{D}$ and $\mathrm{N}$, respectively. Suppose $\mathrm{D}$ and $\mathrm{N}$ both have base period earnings of $v_{0}$ and that the only non-compliant behavior allowed is for $\mathrm{D}$ to claim $b^{0}\left(v_{0}\right)$ or for $\mathrm{N}$ to claim $b^{1}\left(v_{0}\right)$. In this case, we have two potentially unobserved variables that determine treatment: 1) whether a claimant has dependents or not, and 2) whether a claimant "correctly" claims her benefits. We can represent these two variables with a two-dimensional vector $\varepsilon=\left(\varepsilon_{1}, \varepsilon_{2}\right)$. The binary indicator $\varepsilon_{1}$ is equal to 1 if a claimant truly has dependents, whereas $\varepsilon_{2}$ takes four values denoting whether a claimant with base period earnings $v$ is an "always taker" (always claiming $b^{1}(v)$ ), a "never taker" (always receiving $b^{0}(v)$ ), a "complier" (claiming $b^{\varepsilon_{1}}(v)$ ), or a "defier" (claiming $\left.b^{1-\varepsilon_{1}}(v)\right)$. The representation $B=b\left(V, \varepsilon_{1}, \varepsilon_{2}\right)$ effectively captures the treatment assignment mechanism described in this simple example. With suitable definition of $\varepsilon$, it can also be used to allow for many other types of deviations from a deterministic rule. Except for a bounded support assumption similar to that for $U$, we do not need to impose any other restrictions on the distribution of $\varepsilon$. We will use $F_{U, \varepsilon}$ to denote the measure induced by the joint distribution of $U$ and $\varepsilon$.

We also assume that the observed values of $B$ and $V, B^{*}$ and $V^{*}$ respectively, differ from their true values

the two treatment effects in a special case that restricts treatment effect heterogeneity.

${ }^{11}$ See Hahn et al. (2001) for a definition of the fuzzy regression discontinuity design. 
as follows:

$$
\begin{gathered}
V^{*} \equiv V+U_{V} \quad ; \quad B^{*} \equiv B+U_{B} \\
U_{V} \equiv G_{V} \cdot U_{V^{\prime}} \quad ; \quad U_{B} \equiv G_{B} \cdot U_{B^{\prime}},
\end{gathered}
$$

where $U_{V^{\prime}}$ and $U_{B^{\prime}}$ are continuously distributed, and that their joint density conditional on $U$ and $\varepsilon$ is continuous and supported on an arbitrarily large compact rectangle $I_{U_{V^{\prime}}, U_{B^{\prime}}} \subset R^{2} ; G_{V}$ and $G_{B}$ are binary indicators whose joint conditional distribution is given by the four probabilities $\pi^{i j}\left(V, U, \varepsilon, U_{V^{\prime}}, U_{B^{\prime}}\right) \equiv \operatorname{Pr}\left(G_{V}=i, G_{B}=\right.$ $\left.j \mid V, U, \varepsilon, U_{V^{\prime}}, U_{B^{\prime}}\right)$. Note that the errors in the observed values of $V$ and $B$ are assumed to be mixtures of conventional (continuously distributed) measurement error and a point mass at 0 . The random variables $\left(V, U, \varepsilon, U_{V^{\prime}}, U_{B^{\prime}}, G_{V}, G_{B}\right)$ determine $\left(B, B^{*}, V^{*}, Y\right)$, and we observe $\left(B^{*}, V^{*}, Y\right)$.

Assumption 1a. (Regularity) In addition to the conditions in Assumption 1, the support of $\varepsilon$ is bounded: it is a subset of the arbitrarily large compact set $I_{\varepsilon} \subset \mathbb{R}^{k}$.

Assumption 3a. (First stage and non-negligible population at the kink) $b(v, e)$ is continuous on $I_{V, \varepsilon}$ and $b_{1}(v, e)$ is continuous on $\left(I_{V} \backslash\{0\}\right) \times I_{\varepsilon}$. Let $b_{1}^{+}(e) \equiv \lim _{v \rightarrow 0^{+}} b_{1}(v, e), b_{1}^{-}(e) \equiv \lim _{v \rightarrow 0^{-}} b_{1}(v, e)$ and $A_{\varepsilon}=\{e$ : $\left.f_{V \mid \varepsilon=e}(0)>0\right\}$, then $\int_{A_{\varepsilon}} \operatorname{Pr}\left[U_{V}=0 \mid V=0, \varepsilon=e\right]\left|b_{1}^{+}(e)-b_{1}^{-}(e)\right| f_{V \mid \varepsilon=e}(0) d F_{\varepsilon}(e)>0$.

Assumption 4a. (Smooth density) Let $V, U_{V^{\prime}}, U_{B^{\prime}}$ have a well-defined joint probability density function conditional on each $U=u$ and $\varepsilon=e, f_{V, U_{V^{\prime}}, U_{B^{\prime}} \mid U=u, \varepsilon=e}\left(v, u_{B}, u_{V^{\prime}}\right)$. The density function $f_{V, U_{V^{\prime}}, U_{B^{\prime}} \mid U=u, \varepsilon=e}\left(v, u_{B}, u_{V^{\prime}}\right)$ and its partial derivative w.r.t. $v$ are continuous on $I_{V, U_{V^{\prime}}, U_{B^{\prime}}, U, \varepsilon}$.

Assumption 5. (Smooth probability of no measurement error) $\pi^{i j}\left(v, u, e, u_{V^{\prime}}, u_{B^{\prime}}\right)$ and its partial derivative w.r.t. $v$ are continuous on $I_{V, U, \varepsilon, U_{V^{\prime}}, U_{B^{\prime}}}$ for all $i, j=0,1$.

Assumption 6. (Monotonicity) Either $b_{1}^{+}(e) \geq b_{1}^{-}(e)$ for all $e$ or $b_{1}^{+}(e) \leq b_{1}^{-}(e)$ for all $e$.

Extending Assumption 1, Assumption 1a imposes the bounded support assumption for $\varepsilon$ in order to allow the interchange of differentiation and integration. Assumption 3a modifies Assumption 3 and forbids a discontinuity in $b(\cdot, e)$ at the threshold. Analogously to the sharp case discussed in Remark 3 , in the absence of continuity in $b(\cdot, e)$ the RK estimand does not identify a weighted average of the causal effect of interest, $y_{1}$, but the RD estimand does - see subsection A.2 of the Supplemental Appendix for details. Assumption 3a also requires a non-negligible subset of individuals who simultaneously have a non-trivial first stage, have $U_{V}=0$, and have positive probability that $V$ is in a neighborhood of 0 . It is critical that there is a mass point in the distribution of the measurement error $U_{V}$ at 0 . In the absence of such a mass point, we will not observe a kink in the first-stage relationship, and further assumptions must be made about 
the measurement error to achieve identification (as in the case with the RD design). In contrast, there is no need for a mass point in the distribution of $U_{B}$ at 0 , but we simply allow for the possibility here. As shown in Figure 1 for our application below, the majority of the data points $\left(B^{*}, V^{*}\right)$ appear to lie precisely on the benefit schedule, a feature that we interpret as evidence of a mass point at zero in the joint distribution of $\left(U_{V}, U_{B}\right)$. Assumption $3 \mathrm{a}$ can be formally tested by the existence of a first-stage kink in $E\left[B^{*} \mid V^{*}=v^{*}\right]$ as stated in Remark 4 below.

Assumption 4a modifies Assumption 4: for each $U=u$ and $\varepsilon=e$, there is a joint density of $V$ and the measurement error components that is continuously differentiable in $v$. Note that this allows a relatively general measurement error structure in the sense that $V, U_{V^{\prime}}, U_{B^{\prime}}$ can be arbitrarily correlated. Assumption 5 states that the mass point probabilities, while potentially dependent on all other variables, are smooth with respect to $V$.

Assumption 6 states that the direction of the kink is either non-negative or non-positive for the entire population, and it is analogous to the monotonicity condition of Imbens and Angrist (1994). In particular, Assumption 6 rules out situations where some individuals experience a positive kink at $V=0$, but others experience a negative kink at $V=0$. In our application below, where actual UI benefits depend on the (unobserved) number of dependents, this condition is satisfied since the benefit schedules for different numbers of dependents are all parallel.

Proposition 2. In a valid Fuzzy RK Design, that is, when Assumptions 1a, 2, 3a, 4a, 5 and 6 hold:

(a) $\operatorname{Pr}\left(U \leqslant u, \varepsilon \leqslant e \mid V^{*}=v^{*}\right)$ is continuously differentiable in $v^{*}$ at $v^{*}=0 \forall(u, e) \in I_{U, \varepsilon}$.

(b) $\frac{\left.\lim _{v_{0} \rightarrow 0^{+}} \frac{d E\left[Y \mid V^{*}=v^{*}\right]}{d v^{*}}\right|_{v^{*}=v_{0}}-\left.\lim _{v_{0} \rightarrow 0^{-}} \frac{d E\left[Y \mid V^{*}=v^{*}\right]}{d v^{*}}\right|_{v^{*}=v_{0}}}{\left.\lim _{v_{0} \rightarrow 0^{+}} \frac{d E\left[B^{*} \mid V^{*}=v^{*}\right]}{d v^{*}}\right|_{v^{*}=v_{0}}-\left.\lim _{v_{0} \rightarrow 0^{-}} \frac{d E\left[B^{*} \mid V^{*}=v^{*}\right]}{d v^{*}}\right|_{v^{*}=v_{0}}}=\int y_{1}(b(0, e), 0, u) \varphi(u, e) d F_{U, \varepsilon}(u, e)$

where $\varphi(u, e)=\frac{\operatorname{Pr}\left[U_{V}=0 \mid V=0, U=u, \varepsilon=e\right]\left(b_{1}^{+}(e)-b_{1}^{-}(e) \frac{f_{V \mid U=u, \varepsilon=e}(0)}{f_{V}(0)}\right.}{\int \operatorname{Pr}\left[U_{V}=0 \mid V=0, \varepsilon=\omega\right]\left(b_{1}^{+}(\omega)-b_{1}^{-}(\omega)\right) \frac{f_{V \mid \varepsilon=\omega^{(0)}}(0)}{f_{V}(0)} d F_{\varepsilon}(\omega)}$.

The proof is in subsection A.1 of the Supplemental Appendix.

Remark 4. The fuzzy RKD continues to estimate a weighted average of marginal effects of $B$ on $Y$, but the weight is now given by $\varphi(u, e)$. Assumptions $3 \mathrm{a}$ and 6 ensure that the denominator of $\varphi(u, e)$ is nonzero. They also ensure a kink at $v^{*}=0$ in the first-stage relationship between $B^{*}$ and $V^{*}$, as seen from the proof of Proposition 2. It follows that the existence of a first-stage kink serves as a test of Assumptions 3a and 6.

Remark 5. The weight $\varphi(u, e)$ has three components. The first component, $\frac{f_{V \mid U=u, \varepsilon=e}(0)}{f_{V}(0)}$, is analogous to the weight in a sharp RKD and reflects the relative likelihood that an individual of type $U=u, \varepsilon=e$ is 
situated at the kink (i.e., has $V=0$ ). The second component, $b_{1}^{+}(e)-b_{0}^{-}(e)$, reflects the size of the kink in the benefit schedule at $V=0$ for an individual of type $e$. Analogously to the LATE interpretation of a standard instrumental variables setting, the fuzzy RKD estimand upweights types with a larger kink at the threshold $V=0$. Individuals whose benefit schedule is not kinked at $V=0$ do not contribute to the estimand. An important potential difference from a standard LATE setting is that non-compliers may still receive positive weights if the schedule they follow as non-compliers has a kink at $V=0$. Finally, the third component $\operatorname{Pr}\left[U_{V}=0 \mid V=0, U=u, \varepsilon=e\right]$ represents the probability that the assignment variable is correctly measured at $V=0$. Again, this has the intuitive implication that observations with a mismeasured value of the assignment variable do not contribute to the fuzzy RKD estimand. Note that if $\pi^{i j}$ is constant across individuals then this component of the weight is just a constant.

Remark 6. So far we have focused on a continuous treatment variable $B$, but the RKD framework may be applied to estimate the treatment effect of a binary variable as well. As mentioned above, Dong (2013) discusses the identification of the treatment effect within an RD framework where the treatment probability conditional on the running variable is continuous but kinked. Under certain regularity conditions, Dong (2013) shows that the RK estimand identifies the treatment effect at the RD cutoff for the group of compliers. In practice it may be difficult to find policies where the probability of a binary treatment is statutorily mandated to have a kink in an observed running variable. One possibility, suggested by a referee, is that the kinked relationship between two continuous variables $B$ and $V$ may induce a kinked relationship between $T$ and $V$ where $T$ is a binary treatment variable of interest. In this case, we may apply the RK design to measure the treatment effect of $T$. To be more specific, let

$$
\begin{aligned}
Y & =y(T, V, U) \\
T & =1_{\left[T^{*} \geqslant 0\right]} \text { where } T^{*}=t(B, V, \eta) \\
B & =b(V) \text { is continuous in } V \text { with a kink at } V=0 .
\end{aligned}
$$

As an example, $B$ is the amount of financial aid available, which is a kinked function of parental income $V . T^{*}$ is a latent index function of $B, V$, and a one-dimensional error term $\eta$. A student will choose to attend college $(T=1)$ if $T^{*} \geqslant 0$. We are interested in estimating the average returns to college education, an expectation of $y(1, V, U)-y(0, V, U)$. Assuming that $t$ is monotonically increasing in its third argument and that for every $(b, v) \in I_{b(V)} \times I_{V}$ there exists an $n$ such that $t(b(v), v, n)=0$, we can define a continuously 
differentiable function $\tilde{\eta}: I_{b(V)} \times I_{V} \rightarrow \mathbb{R}$ such that $t(b, v, \tilde{\eta}(b, v))=0$ by the implicit function theorem. We show in subsection A.3 of the Supplemental Appendix that under additional regularity conditions, we have the following identification result for the fuzzy RK estimand:

$$
\frac{\left.\lim _{v_{0} \rightarrow 0^{+}} \frac{d E[Y \mid V=v]}{d v}\right|_{v=v_{0}}-\left.\lim _{v_{0} \rightarrow 0^{-}} \frac{d E[Y \mid V=v]}{d v}\right|_{v=v_{0}}}{\left.\lim _{v_{0} \rightarrow 0^{+}} \frac{d E[T \mid V=v]}{d v}\right|_{v=v_{0}}-\left.\lim _{v_{0} \rightarrow 0^{-}} \frac{d E[T \mid V=v]}{d v}\right|_{v=v_{0}}}=\int_{u}[y(1,0, u)-y(0,0, u)] \frac{f_{V, \eta \mid U=u}\left(0, n^{0}\right)}{f_{V, \eta}\left(0, n^{0}\right)} d F_{U}(u)
$$

where $n^{0} \equiv \tilde{\eta}\left(b_{0}, 0\right)$ is the threshold value of $\eta$ when $V=0$ such that $n \geqslant n^{0} \Leftrightarrow T\left(b_{0}, 0, n\right)=1$. The right hand side of equation (4) is similar to that in part (b) of Proposition 1, and the weights reflect the relative likelihood of $V=0$ and $\eta=n^{0}$ for a student of type $U$.

Crucial to the point identification result above is the exclusion restriction that $B$ does not enter the function $y$ as an argument, i.e. that the amount of financial aid does not have an independent effect on future earnings conditional on parental income and college attendance. When this restriction is not met, the RK estimand can be used to bound the effect of $T$ on $Y$ if theory can shed light on the sign of the independent effect of $B$ on $Y$. The details are in subsection A.3 of the Supplemental Appendix.

We can also allow the relationship between $B$ and $V$ to be fuzzy by writing $B=b(V, \varepsilon)$ and introducing measurement error in $V$ as above. Similar to Proposition 2, we show that the fuzzy RK estimand still identifies a weighted average of treatment effect under certain regularity assumptions. The weights are similar to those in Proposition 2, and the exact expression is in the Supplemental Appendix.

\subsection{Testable Implications of the RKD}

In this section we formalize the testable implications of a valid RK design. Specifically, we show that the key smoothness conditions given by Assumptions 4 and 4 a lead to two strong testable predictions. The first prediction is given by the following corollary of Propositions 1 and 2:

Corollary 1. In a valid Sharp RKD, $f_{V}(v)$ is continuously differentiable in $v$. In a valid Fuzzy $R K D, f_{V^{*}}\left(v^{*}\right)$ is continuously differentiable in $v^{*}$.

The key identifying assumption of the sharp RKD is that the density of $V$ is sufficiently smooth for every individual. This smoothness condition cannot be true if we observe either a kink or a discontinuity in the density of $V$. That is, evidence that there is "deterministic sorting" in $V$ at the kink point implies a violation of the key identifying sharp RKD assumption. This is analogous to the test of manipulation of the assignment variable for RD designs, discussed in McCrary (2008). In a fuzzy RKD, both Assumption 4a, the 
smooth-density condition, and Assumption 5, the smooth-probability-of-no-measurement-error condition, are needed to ensure the smoothness of $f_{V^{*}}$ (see the proof of Lemma 5), and a kink or a discontinuity in $f_{V^{*}}$ indicates that either or both of the assumptions are violated.

The second prediction presumes the existence of data on "baseline characteristics" - analogous to characteristics measured prior to treatment assignment in an idealized randomized controlled trial - that are determined prior to $V$.

Assumption 8. There exists an observable random vector, $X=x(U)$ in the sharp design and $X=x(U, \varepsilon)$ in the fuzzy design, that is determined prior to $V . X$ does not include $V$ or $B$, since it is determined prior to those variables.

In conjunction with our basic identifying assumptions, this leads to the following prediction:

Corollary 2. In a valid Sharp RKD, if Assumption 8 holds, then $\frac{d \operatorname{Pr}[X \leq x \mid V=v]}{d v}$ is continuous in $v$ at $v=0$ for all $x$. In a valid Fuzzy RKD, if Assumption 8 holds, then $\frac{d \operatorname{Pr}\left[X \leq x \mid V^{*}=v^{*}\right]}{d v^{*}}$ is continuous in $v^{*}$ at $v^{*}=0$ for all $x$.

The smoothness conditions required for a valid RKD imply that the conditional distribution function of any predetermined covariates $X$ (given $V$ or $V^{*}$ ) cannot exhibit a kink at $V=0$ or $V^{*}=0$. Therefore, Corollary 2 can be used to test Assumption 4 in a sharp design and Assumption 4a and 5 jointly in a fuzzy design. This test is analogous to the simple "test for random assignment" that is often conducted in a randomized trial, based on comparisons of the baseline covariates in the treatment and control groups. It also parallels the test for continuity of $\operatorname{Pr}[X \leq x \mid V=v]$ emphasized by Lee (2008) for a regression discontinuity design. Importantly, however, the assumptions for a valid RKD imply that the derivatives of the conditional expectation functions (or the conditional quantiles) of $X$ with respect to $V$ (or $V^{*}$ ) are continuous at the kink point - a stronger implication than the continuity implied by the sufficient conditions for a valid RDD.

\section{Nonparametric Estimation and Inference in a Regression Kink Design}

In this section, we review the theory of estimation and inference in a regression kink design. We assume that estimation is carried out via local polynomial regressions. For a sharp RK design, the first stage relationship $b(\cdot)$ is a known function, and we only need to solve the following least squares problems

$$
\min _{\left\{\tilde{\beta}_{j}^{-}\right.} \sum_{i=1}^{n^{-}}\left\{Y_{i}^{-}-\sum_{j=0}^{p} \tilde{\beta}_{j}^{-}\left(V_{i}^{-}\right)^{j}\right\}^{2} K\left(\frac{V_{i}^{-}}{h}\right)
$$




$$
\min _{\left\{\tilde{\beta}_{j}^{+}\right\}} \sum_{i=1}^{n^{+}}\left\{Y_{i}^{+}-\sum_{j=0}^{p} \tilde{\beta}_{j}^{+}\left(V_{i}^{+}\right)^{j}\right\}^{2} K\left(\frac{V_{i}^{+}}{h}\right)
$$

where the - and + superscripts denote quantities in the regression on the left and right side of the kink point respectively, $p$ is the order of the polynomial, $K$ the kernel, and $h$ the bandwidth. Since $\kappa_{1}^{+}=\lim _{v \rightarrow 0^{+}} b^{\prime}(v)$ and $\kappa_{1}^{-}=\lim _{v \rightarrow 0^{-}} b^{\prime}(v)$ are known quantities in a sharp design, the sharp RKD estimator is defined as

$$
\hat{\tau}_{S R K D}=\frac{\hat{\beta}_{1}^{+}-\hat{\beta}_{1}^{-}}{\kappa_{1}^{+}-\kappa_{1}^{-}} .
$$

In a fuzzy RKD, the first stage relationship is no longer deterministic. We need to estimate the first-stage slopes on two sides of the threshold by solving ${ }^{12}$

$$
\begin{gathered}
\min _{\tilde{\kappa}_{j}^{-}} \sum_{i=1}^{n^{-}}\left\{B_{i}^{-}-\sum_{j=0}^{p} \tilde{\kappa}_{j}^{-}\left(V_{i}^{-}\right)^{j}\right\}^{2} K\left(\frac{V_{i}^{-}}{h}\right) \\
\min _{\tilde{\kappa}_{j}^{+}} \sum_{i=1}^{n^{+}}\left\{B_{i}^{+}-\sum_{j=0}^{p} \tilde{\kappa}_{j}^{+}\left(V_{i}^{+}\right)^{j}\right\}^{2} K\left(\frac{V_{i}^{+}}{h}\right) .
\end{gathered}
$$

The fuzzy RKD estimator $\hat{\tau}_{F R K D}$ can then be defined as

$$
\hat{\tau}_{F R K D}=\frac{\hat{\beta}_{1}^{+}-\hat{\beta}_{1}^{-}}{\hat{\kappa}_{1}^{+}-\hat{\kappa}_{1}^{-}} .
$$

Lemma A1 and A2 of Calonico et al. (Forthcoming) establish the asymptotic distributions of the sharp and fuzzy RKD estimators, respectively. It is shown that under certain regularity conditions the estimators obtained from local polynomial regressions of order $p$ are asymptotically normal:

$$
\begin{aligned}
& \sqrt{n h^{3}}\left(\hat{\tau}_{S R K D, p}-\tau_{S R K D}-h^{p} \rho_{S R K D, p}\right) \Rightarrow N\left(0, \Omega_{S R K D, p}\right) \\
& \sqrt{n h^{3}}\left(\hat{\tau}_{F R K D, p}-\tau_{F R K D}-h^{p} \rho_{F R K D, p}\right) \Rightarrow N\left(0, \Omega_{F R K D, p}\right)
\end{aligned}
$$

where $\rho$ and $\Omega$ denote the asymptotic bias and variance, respectively. ${ }^{13}$ Given the identification assumptions above, one expects the conditional expectation of $Y$ given $V$ to be continuous at the threshold. A natural question is whether imposing continuity in estimation (as opposed to estimating separate local polynomials

\footnotetext{
${ }^{12}$ We omit the asterisk in $B^{*}$ and $V^{*}$ notations in the fuzzy design to ease exposition.

${ }^{13}$ In categorizing the asymptotic behavior of fuzzy estimators, both Card et al. (2012) and Calonico et al. (Forthcoming) assume that the researcher observes the joint distribution $(Y, B, V)$. In practice, there may be applications where $(B, V)$ is observed in one data source whereas $(Y, V)$ is observed in another, and the three variables do not appear in the same data set. We investigate the two-sample estimation problem in subsection B.1 of the Supplemental Appendix.
} 
on either side of the threshold) may affect the asymptotic bias and variance of the kink estimator. Card et al. (2012) shows that when $K$ is uniform the asymptotic variances are not affected by imposing continuity. A similar calculation reveals that the asymptotic biases are not affected either.

When implementing the RKD estimator in practice, one must make choices for the polynomial order $p$, kernel $K$ and bandwidth $h$. In the RD context where the quantities of interest are the intercept terms on two sides of the threshold, Hahn et al. (2001) propose local linear $(p=1)$ over local constant $(p=0)$ regression because the former leads to a smaller order of bias $\left(O_{p}\left(h^{2}\right)\right)$ than the latter $\left(O_{p}(h)\right)$. Consequently, the local linear model affords the econometrician a sequence of bandwidths that shrinks at a slower rate, which in turn delivers a smaller order of the asymptotic mean-squared error (MSE). The same logic would imply that a local quadratic $(p=2)$ should be preferred to local linear $(p=1)$ in estimating boundary derivatives in the RK design. As we argue in Card et al. (2014), however, arguments based solely on asymptotic rates cannot justify $p=1$ as the universally preferred choice for RDD or $p=2$ as the universally preferred choice for RKD. Rather, the best choice of $p$ in the mean squared error sense depends on the sample size and the derivatives of the conditional expectation functions, $E[Y \mid V=v]$ and $E[B \mid V=v]$, in the particular data set of interest. In Card et al. (2014), we propose two methods for picking the polynomial order for interested empiricists: 1) evaluate the empirical performance of the alternative estimators using simulation studies of DGP's closely based on the actual data; 2) estimate the asymptotic mean squared error (AMSE) and compare it across alternative estimators. Using these methods we argue in section 4 below that the local linear estimator is a more sensible choice than the local quadratic for the Austrian UI data we study.

For the choice of $K$ we adopt a uniform kernel following Imbens and Lemieux (2008) and the common practice in the RD literature. The results are similar when the boundary optimal triangular kernel (c.f. Cheng et al. (1997)) is used.

For the bandwidth choice $h$, we use and extend existing selectors in the literature. Imbens and Kalyanaraman (2012) propose an algorithm to compute the MSE-optimal RD bandwidth. Building on Imbens and Kalyanaraman (2012), Calonico et al. (Forthcoming) develop an optimal bandwidth algorithm for the estimation of the discontinuity in the $v$-th derivative, which contains $\operatorname{RKD}(v=1)$ as a special case. ${ }^{14}$

We examine alternatives to the direct analogs of the default IK and the CCT bandwidths for RKD, addressing two specific issues that are relevant for our setting. First, both bandwidth selectors involve a

\footnotetext{
${ }^{14}$ The optimal bandwidth in Calonico et al. (Forthcoming) is developed for the unconstrained RKD estimator, i.e. without imposing continuity in the conditional expectation of $Y$, but the bandwidth is also optimal for the constrained RKD estimator because it has the same asymptotic distribution as stated above.
} 
regularization term, which reflects the variance in the bias estimation and guards against large bandwidths. While IK and CCT argue that the regularized bandwidth selector performs well for several well-known regression discontinuity designs, we find that the RK counterparts of these regularized selectors yield bandwidths that tend to be too small in our empirical setting. Since omitting the regularization term does not affect the asymptotic properties of the bandwidth selector, we also investigate the performance of IK and CCT bandwidth selectors without the regularization term. Second, the CCT bandwidth is asymptotically MSE-optimal for the reduced-form kink in a fuzzy design, even though the fuzzy estimator $\hat{\tau}_{F R K D}$ defined in (9) is the main object of interest. Based on the asymptotic theory in Calonico et al. (Forthcoming), we propose fuzzy analogs of the IK and CCT bandwidths that are optimal for $\hat{\tau}_{F R K D}$ and state their asymptotic properties - see the subsection B.2 of Supplemental Appendix for details.

A complication of using the optimal bandwidth is that the asymptotic bias is in general nonzero. As a result, conventional confidence intervals that ignore the bias may not have correct coverage rates. Calonico et al. (Forthcoming) offer a solution by deriving robust confidence intervals for the RD and RK estimands that account for this asymptotic bias. For an RK design, they first estimate the asymptotic bias $\rho_{p}$ of a $p$-th order local polynomial estimator $\hat{\tau}_{p}$ by using a $q$-th order local polynomial regression $(q \geqslant p+1)$ with pilot bandwidth $h_{q}$, then estimate the variance $\operatorname{var}_{p}^{b c}$ of the bias-corrected estimator $\hat{\tau}_{p}^{b c} \equiv \hat{\tau}_{p}-h^{p} \hat{\rho}_{p}$, by accounting for the sampling variation in both $\hat{\tau}_{p}$ and $h^{p} \hat{\rho}_{p} \cdot{ }^{15}$ Finally, they construct a robust $95 \%$ confidence interval as $\hat{\tau}_{p}^{b c} \pm 1.96 \sqrt{\operatorname{var}_{p}^{b c}}$. Using Monte Carlo simulations, Calonico et al. (Forthcoming) demonstrate that the confidence intervals constructed using their bias-corrected procedure perform well in RDD's, and that the associated coverage rates are robust to different choices of $h .{ }^{16}$

In the following section, we present a variety of alternative estimates of the behavioral effect of higher benefits on the duration of joblessness. We investigate the performance of these alternative estimators using simulated DGP's that are closely based on our actual data. The candidates include local linear and local quadratic estimators with several bandwidth selectors - default CCT, CCT without regularization, Fuzzy CCT, Fuzzy IK and the FG bandwidth. ${ }^{17}$ We report uncorrected RKD estimates and the associated

\footnotetext{
${ }^{15} \mathrm{~A}$ crucial assumption in estimating $\operatorname{var}_{p}^{b c}$ is that the pilot bandwidth $h_{q}$ and the optimal bandwidth $h$ have the same shrinkage rate, i.e. $\frac{h_{q}}{h} \rightarrow \rho \in(0, \infty)$ as $n \rightarrow \infty$.

${ }^{16}$ In a related study, Ganong and Jäger (2014) raise concerns about the sensitivity of the RKD estimates when the relationship between the running variable and the outcome is highly nonlinear. They propose a permutation test to account for the estimation bias. We perform the test on our data and discuss the details in subsection B.3 of the Supplemental Appendix.

${ }^{17}$ See Card et al. (2012) for the definition of the FG bandwidth. We apply the same logic to derive the pilot FG bandwidth for bias estimation.
} 
(conventional) sampling errors associated with each polynomial order and bandwidth choice, as well as bias-corrected estimates and the associated robust confidence intervals suggested by Calonico et al. (Forthcoming). ${ }^{18}$

\section{The Effect of UI Benefits on the Duration of Joblessness}

In this section, we use a fuzzy RKD approach to estimate the effect of higher unemployment benefits on the duration of joblessness among UI claimants in Austria. The precise magnitude of the disincentive effect of UI benefits is of substantial policy interest. As shown by Baily (1978), for example, an optimal unemployment insurance system trades off the moral hazard costs of reduced search effort against the risk-sharing benefits of more generous payments to the unemployed. ${ }^{19}$ Obtaining credible estimates of this effect is difficult, however, because UI benefits are determined by previous earnings, and are likely to be correlated with unobserved characteristics of workers that affect both wages and the expected duration of unemployment. Since the UI benefit formula in Austria has both a minimum and maximum, a regression kink approach can provide new evidence on the impact of higher UI benefits at two different points in the benefit schedule. We begin with a brief discussion of a job search model that we use to frame our analysis. We then describe the benefit system in Austria, our data sources, and our main results.

\subsection{Theoretical background}

In a standard search model, higher UI benefits reduce the incentives for search and raise the reservation wage, leading to increases in the expected duration of joblessness. Higher benefits can also affect the equilibrium distribution of wages. Christensen et al. (2005), for example, derive the equilibrium distribution of wages, given a fixed UI benefit and a latent distribution of wage offers. In their model, a twice continuously differentiable distribution function for wage offers ensures that distribution of wages among newly laid-off workers is twice continuously differentiable. In section $\mathrm{C}$ of the Supplemental Appendix we extend this model to incorporate a UI benefit schedule that is linear in the previous wage up to some maximum $T^{\max }$. In this case the value function of unemployed workers is increasing in their previous wage with a kink at $T^{\max }$. Likewise, the value function associated with a job paying a wage $w$ has a kink at $w=T^{\max }$, reflecting

\footnotetext{
${ }^{18}$ Even though $\hat{\tau}_{p}^{b c}$ is not consistent under the CCT asymptotic assumptions regarding the shrinkage rate of $h_{q}$ and $h$, it may still be informative to report its value and shed light on the direction and magnitude of the estimated bias.

${ }^{19}$ The original analysis in Baily (1978) has been generalized to allow for liquidity constraints (Chetty (2010)) and variable takeup (Kroft (2008)).
} 
the kink in the option value of UI benefits when the job ends. This kink causes a kink in the relationship between wages and on-the-job search effort which leads to a kink in the density of wages at $T^{\max }$ (see the Supplemental Appendix for details). Assuming a constant rate of job destruction there is a similar kink in the density of previous wages among job losers. Such a kink - at precisely the threshold for the maximum benefit rate - violates the smooth density condition (i.e., Assumptions 4/4a) necessary for a valid regression kink design based on the change in the slope of the benefit function at $T^{\max } \cdot{ }^{20}$ Nevertheless, we also show that if workers have some uncertainty about the location of the kink in future UI benefits, the equilibrium density of previous wages among job losers will be smooth at $T^{\max }$.

Given these theoretical possibilities, it is important to examine the actual distribution of pre-displacement wages among job seekers and test for the presence of kinks around the minimum and maximum benefit thresholds, as well as for kinks in the conditional distributions of predetermined covariates. While we do not necessarily expect to find kinks in our setting (given the difficulty of forecasting future benefit schedules in Austria), a kink could exist in other settings where minimum or maximum UI benefits are often fixed for several years at the same nominal value.

\subsection{The Unemployment Insurance System in Austria}

Job losers in Austria who have worked at least 52 weeks in the past 24 months are eligible for UI benefits, with a rate that depends on their average daily earnings in the "base year" for their benefit claim, which is either the previous calendar year or the second most recent year. The daily UI benefit is calculated as $55 \%$ of net daily earnings, subject to a maximum benefit level that is adjusted each year. Claimants with dependent family members are eligible for supplemental benefits based on the number of dependents. There is also a minimum benefit level for lower-wage claimants, subject to the proviso that total benefits cannot exceed $60 \%$ (for a single individual) or $80 \%$ (for a claimant with dependents) of base year net earnings.

These rules create a piecewise linear relationship between base year earnings and UI benefits that depends on the Social Security and income tax rates as well as the replacement rate and the minimum and maximum benefit amounts. To illustrate, Figure 1 plots actual daily UI benefits against annual base year earnings for a sample of UI claimants in 2004. The high fraction of claimants whose observed UI benefits

\footnotetext{
${ }^{20}$ In the model as written, all workers are identical; hence a kink in the density of wages at $T^{\text {max }}$ will not actually invalidate an RK design. More realistically, however, workers differ in their cost of search (and in other dimensions), and the kink at $T^{\text {max }}$ is larger for some types than others, causing a discontinuity in the conditional distribution of unobserved heterogeneity at $T^{\max }$ that leads to bias in an RKD.
} 
are exactly equal to the amount predicted by the formula leads to a series of clearly discernible lines in the figure, though there are also many observations scattered above and below these lines. ${ }^{21}$ Specifically, in the middle of the figure there are 5 distinct upward-sloping linear segments, corresponding to claimants with 0 , 1, 2, 3, or 4 dependents. These schedules all reach an upper kink point at the maximum benefit threshold (which is shown in the graph by a solid vertical line). At the lower end, the situation is more complicated: each of the upward-sloping segments reaches the minimum daily benefit at a different level of earnings, reflecting the fact that the basic benefit includes family allowances, but the minimum does not. Finally, among the lowest-paid claimants the benefit schedule becomes upward-sloping again, with two major lines representing single claimants (whose benefit is $60 \%$ of their base earnings, net of taxes) and those with dependents (whose benefits are $80 \%$ of their net base year earnings). ${ }^{22}$

Our RKD analysis exploits the kinks induced by the minimum and maximum benefit levels. Since we do not observe the number of dependents claimed by a job loser, we adopt a fuzzy RKD approach in which the number of dependents is treated as an unobserved determinant of benefits. This does not affect the location of the "top kink" associated with the maximum benefit, since claimants with different numbers of dependents all have the same threshold earnings level $T^{\max }$ for reaching the maximum. For the "bottom kink" associated with the minimum benefit, we define $T^{\min }$ as the kink point for a single claimant: this is the level of annual earnings shown in the figure by a solid vertical line. To the right of $T^{\min }$, the benefit schedules for all claimant groups are upward-sloping. To the left, benefits for claimants with no dependents are constant, whereas benefits for claimants with dependents continue to fall. Thus we expect to measure a kink in the average benefit function at $T^{\min }$ that is proportional to the fraction of claimants with no dependents. We limit our analysis to claimants whose earnings are high enough to avoid the "subminimum" portion of the benefit schedule: this cutoff is shown by the dashed line on the left side of Figure 1. We also focus on claimants whose annual earnings are below the Social Security contribution cap, since earnings above this level are censored. This cutoff is shown by the dashed line on the right side of Figure 1.

\footnotetext{
${ }^{21}$ These are attributable to some combination of errors in the calculation of base year earnings (due to errors in the calculation of the claim start date, for example), errors in the Social Security earnings records that are overridden by benefit administrators, and mis-reported UI benefits. Similar errors have been found in many other settings - e.g., Kapteyn and Ypma (2007).

${ }^{22}$ The line for low-earning single claimants actually bends, reflecting the earnings threshold at which a single claimant begins paying income taxes.
} 


\subsection{Data and Analysis Sample}

Our data are drawn from the Austrian Social Security Database (ASSD), which records employment and unemployment spells on a daily basis for all individuals employed in the Austrian private sector (see Zweimüller et al. (2009)). The ASSD contains information on starting and ending dates of spells and earnings (up to the Social Security contribution cap) received by each individual from each employer in a calendar year. We merge the ASSD with UI claims records that include the claim date, the daily UI benefit actually received by each claimant, and the duration of the benefit spell. We use the UI claim dates to assign the base calendar year for each claim and then calculate base year earnings for each claim, which is the observed assignment variable for our RKD analysis (i.e., $V^{*}$ in the notation of section 2 ). In addition, we observe the claimant's age, gender, education, marital status, job tenure, and industry. Our main outcome variable is the time between the end of the old job and the start of any new job (which we censor at 1 year).

Our analysis sample includes claimants from 2001-2012 with at least one year of tenure on their previous job who initiated their claim within four weeks of the job ending date (eliminating job quitters, who face a four-week waiting period). We drop people with zero earnings in the base year, claimants older than 50, and those whose earnings are above the Social Security earnings cap or so low that they fall on the "subminimum" portion of the benefit schedule. We pool observations from different years as follows. First, we divide the claimants in each year into two (roughly) equal groups based on their gross base year earnings: those below the 50th percentile are assigned to the "bottom kink" sample, while those above this threshold are assigned to the "top kink" sample. Since earnings have a right-skewed distribution, the cutoff threshold is closer to $T^{\min }$ than $T^{\max }$, implying a narrower support for our observed assignment variable $V^{*}$ (observed annual base year earnings) around the bottom kink than the top kink. Next we re-center base year earnings for observations in the bottom kink subsample around $T^{\text {min }}$, and base year earnings for those in the top kink subsample around $T^{\max }$, so both kinks occur at $V^{*}=0$. Finally, we pool the yearly re-centered subsamples into bottom and top kink samples, yielding about 275,000 observations in each sample.

Table 1 reports basic summary statistics for the bottom and top kink samples. Mean base year earnings for the bottom kink group are about $€ 22,000$, with a relatively narrow range of variation (standard deviation $=€ 2,800$ ), while mean earnings in the top kink group are higher (mean $=€ 34,000)$ and more dispersed (standard deviation $=€ 6,700$ ). Mean daily UI benefits are $€ 25.2$ for the bottom kink group (implying an annualized benefit of $€ 9,200$, about $44 \%$ of $T^{\text {min }}$ ), while mean benefits for the top kink sample are $€ 33.5$ 
(implying an annualized benefit of $€ 12,300$, about $28 \%$ of $T^{\max }$ ). Claimants in the bottom kink sample are more likely to be female, are a little younger, less likely to be married, more likely to have had a blue-collar occupation, and are less likely to have post-secondary education. Despite the differences in demographic characteristics and mean pay, the means of the main outcome variable are quite similar in the two samples: the average duration of joblessness is around 150 days. Only about 10 percent of claimants exhaust their regular UI benefits.

A key assumption for valid inference in an RK design is that the density of the assignment variable (in our case, base year earnings) is smooth at the kink point. Figures $2 \mathrm{a}$ and $2 \mathrm{~b}$ show the frequency distributions of base year earnings in our two subsamples, using 100-Euro bins for the bottom kink sample and 300-Euro bins for the top kink sample (each with about 4,200 observations per bin). While the histograms look quite smooth, we tested this more formally by fitting a series of polynomial models that allow the first and higherorder derivatives of the binned density function to jump at the kink point. ${ }^{23}$ We test for a kink by testing for a jump in the linear term of the polynomial at the kink. Appendix Table 1 shows the goodness of fit and Akaike model selection statistics for polynomial models of order 2, 3, 4, or 5, as well as the estimated kinks at $T^{\min }$ and $T^{\max }$. We show the fitted values from the models with the lowest Akaike criterion - a 3rd order model for the bottom kink sample and a 4th order model for the top kink sample - in Figures 2a and 2b. In both cases the fitted densities appear to be quite smooth.

\subsection{Graphical Overview of the Effect of Kinks in the UI Benefit Schedule}

As a starting point for our RKD analysis, Figures 3 and 4 show the relationships between base year earnings and actual UI benefits around the bottom and top kinks. We plot the data using the same bin sizes as in Figures $2 \mathrm{a}$ and $2 \mathrm{~b}^{24}$ The figures show clear kinks in the empirical relationship between average benefits and base year earnings, with a sharp increase in slope as earnings pass through the lower threshold $T^{\min }$ and a sharp decrease as they pass through the upper threshold $T^{\max } \cdot{ }^{25}$ Figures 5 and 6 present parallel figures for the mean log time to the next job. These figures also show discernible kinks, though there is clearly more variability in the relationship with base year earnings.

\footnotetext{
${ }^{23}$ We use a minimum chi-squared objective, which Lindsay and Qu (2003) show can be interpreted as an optimally weighted minimum distance objective for the multinomial distribution of histogram frequencies.

${ }^{24}$ See Calonico et al. (2014a) for nonparametric procedures for picking the bin size in RD-type plots.

${ }^{25}$ The slopes in the mean benefit functions to the left of $T^{\min }$, and to the right of $T^{\max }$ are mainly attributable to family allowances. Moving left from $T^{\mathrm{min}}$ the average number of dependent allowances is falling, as claimants with successively higher numbers of dependents hit the minimum benefit level (see Figure 1). Likewise, moving right from $T^{\text {max }}$, the average number of allowances is rising, reflecting a positive correlation between earnings and family size.
} 
Given the relatively short duration of UI benefits in our sample (20 - 39 weeks), it is also interesting to look at the probability a claimant exhausts benefits. Appendix Figure 1a shows this probability around the bottom kink. The discrete increase in the slope with respect to base year earnings suggests that higher UI benefits increase the probability of exhaustion. Appendix Figure 1b presents a parallel graph around the top kink. The exhaustion probability exhibits a kink in the expected direction, though as with our main outcome variable, the probabilities are relatively noisy in the range of earnings just above $T^{\max }$.

Finally, we examine the patterns of the predetermined covariates around $T^{\min }$ and $T^{\max }$. Appendix Figures 2 and 3 show the conditional means of four main covariates around the two kink points: age, gender, blue-collar occupation, and an indicator for whether the claimant had been recalled to the previous job. ${ }^{26}$ The graphs show some evidence of non-smoothness in the conditional means of the covariates in the bottom kink sample, particularly for claimant age. To increase the power of this analysis we constructed a "covariate index" - the predicted duration of joblessness from a simple linear regression model relating the log of time to next job to a total of 59 predetermined covariates, including gender, occupation, age, previous job tenure, quintile of the previous daily wage, industry, region, year of the claim, previous firm size, and the recall rates of the previous employer. ${ }^{27}$ This estimated covariate index function can be interpreted as the best linear prediction of mean log time to next job given the vector of predetermined variables.

Figures 7 and 8 plot the mean values of the estimated covariate indices around the top and bottom kinks. Visually, the predicted time to next job appears to evolve relatively smoothly through both the top and bottom kinks. In the next subsection, we provide a more formal comparison of the estimated slopes of the conditional mean functions for the covariate indices.

\subsection{RKD Estimation Results}

\subsubsection{Reduced Form Kinks in Assignment and Outcome Variables}

Table $2 \mathrm{a}$ presents reduced form estimates of the kinks in our endogenous policy variable (log daily benefits) and our main outcome variable (log of time to next job) around $T^{\min }$ and $T^{\max }$. For each variable we show results using three different bandwidth selection procedures: the default CCT procedure; the CCT bandwidth selection procedure without regularization; and the FG bandwidth. We show the estimated kink arising from

\footnotetext{
${ }^{26}$ Many seasonal jobs in Austria lay off workers at the end of the season and rehire them again at the start of the next season. Having been recalled from unemployment to the recently lost job is a good indicator that the present spell may end with recall to that job again - see Del Bono and Weber (2008).

${ }^{27}$ We fit a single prediction model using the pooled bottom kink and top kink samples.
} 
each selected bandwidth, as well as the corresponding bias-corrected estimate and the associated robust $95 \%$ confidence interval. ${ }^{28}$ We present estimates from local linear models in columns 1 and 3, and from local quadratic models in columns 2 and 4.

Despite the strong visual evidence of kinks in the benefit formula in Figures 3 and 4, an examination of the estimated "first stage" kinks in Panel A of Table 2 suggests that not all the procedures yield statistically significant kink estimates. In particular, the default CCT bandwidth selector chooses relatively small bandwidths for the local linear model and yields an insignificant estimate of the bottom kink $(t=1.7)$ and only a marginally significant estimate of the top kink $(t=2.1)$. The corresponding bias-corrected kink estimates are substantially less precise, with sampling errors about $40 \%$ larger than the uncorrected estimates. Although the default CCT procedure chooses somewhat larger bandwidths for the local quadratic models, this is offset by the difficulty of precisely estimating the slopes on either side of the kink point once the quadratic terms are included, and neither the estimated bottom kink nor the estimated top kink in the quadratic models is close to significant. As with the local linear models, the corresponding bias-corrected quadratic kink estimates are even less precise, with very wide confidence intervals.

Relative to the default CCT bandwidth selector, the CCT selector without regularization yields substantially larger bandwidths - over 2 times larger for the local linear models, and 30-50\% larger for the local quadratic models. These larger bandwidths yield uncorrected first stage kink estimates from the local linear models that are relatively precise ( $t>10$ for the bottom kink sample, $t=6$ for the top kink sample). The estimated kinks from the local quadratic estimates, however, are still relatively noisy, as are the bias-corrected estimates from either the local linear or local quadratic models. By comparison, the bandwidths selected by the FG procedure are relatively large and deliver seven significant first stage estimates in eight cases. Interestingly, a comparison of the uncorrected and bias-corrected estimates from the FG selection procedure suggests that the biases associated with the naive FG bandwidth choice are relatively small, except in the case of the local quadratic estimate for the top kink.

Turning to the reduced form outcome models in Panel B, the estimated kinks in the duration of joblessness are less precisely estimated than the kinks in log benefits. Again, the default CCT bandwidth selector chooses relatively small bandwidths and yields very noisy estimates of the kink. The bandwidths under the CCT procedure without the regularization term are substantially larger, and yield marginally significant

\footnotetext{
${ }^{28}$ Robust confidence intervals and the CCT bandwidths are obtained based on a variant of the Stata package described in Calonico et al. (in press) with the nearest-neighbor variance estimator, which we also use in the simulations below. Using the CCT Stata package generates very similar empirical estimates.
} 
estimated kinks in the outcome variable from the local linear models. The FG bandwidths are even larger, and they yield estimated kinks that are significant or marginally significant in both the linear and quadratic models. The bias-corrected estimates are in all but one case insignificant, however, reflecting the additional uncertainty associated with the bias correction term. ${ }^{29}$ Interestingly, the bias-corrected local linear estimates for the bottom and top kink are both larger in absolute value than the corresponding uncorrected estimates, suggesting that the uncorrected estimates may be conservative. Finally, as we found with the first stage kink estimates, the local quadratic estimates in the reduced form model are quite imprecise, and the bias-corrected local quadratic models are essentially uninformative.

In finite samples, the use of higher order polynomial models and bias correction may come at the cost of an increase in variance relative to lower-order uncorrected models. In the remainder of this section we examine the tradeoff between bias and variance associated with the CCT bias correction procedure. We defer a discussion of the polynomial order choice to subsection 4.5.3. As mentioned in section 3, the intent of CCT's bias correction is to eliminate the bias in the $p$-th order polynomial estimator $\hat{\tau}_{p}$ by subtracting the estimated asymptotic bias, $h^{p} \hat{\rho}_{p}$. The cost of bias correction, however, is that the bias term is imprecisely estimated, leading to a potential increase in the overall variance of the corrected estimator $\hat{\tau}_{p}^{b c}$ relative to the uncorrected estimator $\hat{\tau}_{p}{ }^{30}$ The usual metric for trading off bias and variance is the (asymptotic) mean squared error of the estimator, which is the sum of its squared bias and its variance. By Lemma A1 and Theorem A1 of Calonico et al. (Forthcoming), the asymptotic mean squared errors of $\hat{\tau}_{p}$ and $\hat{\tau}_{p+1}^{b c}$ are $\operatorname{AMSE}\left(\hat{\tau}_{p}\right)=\left(h^{p} \rho_{p}\right)^{2}+o_{p}\left(h^{2 p}\right)+\operatorname{var}\left(\hat{\tau}_{p}\right)$ and $\operatorname{AMSE}\left(\hat{\tau}_{p}^{b c}\right)=o_{p}\left(h^{2 p}\right)+\operatorname{var}_{p}^{b c}$. It follows that the change in the AMSE associated with bias correction is asymptotically $-\left(h^{p} \rho_{p}\right)^{2}+\operatorname{var}_{p}^{b c}-\operatorname{var}\left(\hat{\tau}_{p}\right)$.

In Table $2 \mathrm{~b}$, we report the estimated bias $h^{p} \hat{\rho}_{p}$, its square, and the change in estimated variance, $\widehat{\operatorname{var}}_{p}^{b c}-$ $\widehat{\operatorname{var}}\left(\hat{\tau}_{p}\right)$, for the first stage and reduced form estimators presented in Table 2a. The increase in variance is larger than the estimated squared bias for both the local linear and local quadratic estimates using either the default CCT bandwidth selector or the alternative version that ignores the regularization term. This is also the case for the FG bandwidth in the bottom kink sample. In the top kink sample, however, the estimated bias for the FG bandwidth is quite large, and bias correction appears to decrease the AMSE. This suggests that bias correction could be important for estimators based on the FG bandwidth in the top kink sample. At

\footnotetext{
${ }^{29}$ The one exception is the bias-corrected local linear estimate for the top kink sample. In this case, the robust confidence interval is relatively wide but the bias-corrected point estimate is also relatively large in magnitude.

${ }^{30}$ Remark 5 of Calonico et al. (Forthcoming) states that the variance of $\hat{\tau}_{p}^{b c}$ is smaller than that of $\hat{\tau}_{p}$ for a large $n$, but this asymptotic advantage may not materialize in a given finite sample and does not appear to hold in our samples.
} 
the same time, since the bias term is estimated, it may deviate from the actual bias. So in section 4.5.3, we evaluate the performance of the various estimators in Monte Carlo simulations using DGP's approximating our data, where we can directly obtain the mean squared errors without having to estimate the bias.

\subsubsection{Kinks in Conditional Means of Predetermined Covariates}

As discussed in subsection 2.3, a key implication of the smooth density assumption underlying a valid RK design is that the conditional distributions of any pre-determined covariates should evolve smoothly around the kink points in the observed running variable. In the context of our UI example, this means that the conditional means of all pre-determined claimant characteristics should vary smoothly with base-period earnings around the bottom and top kink points. Table 3 presents tests of this smoothness prediction for the covariate index introduced in Figures 7 and 8, as well as for the four main sub-components of this index. We show estimated kinks from local linear and local quadratic models using the FG bandwidth selector, as well as the corresponding bias-corrected kink estimates (and robust 95\% confidence intervals).

For the bottom kink sample (Panel A), the estimates point to some reason for concern. In particular, the conditional mean of the predicted mean of joblessness exhibits a positive kink at $T^{\min }$ about $30 \%$ as large as the kink in actual log time to next job (comparing the estimated kink of 0.9 for predicted log time to the estimate of 3.0 for log actual time in Table 2a from a local linear model using the FG bandwidth). Looking at the individual covariates, there are relatively large kinks in the conditional means of age and the indicator for blue collar status. These kinks are visually evident in Appendix Figures 2a and 2c and suggest that the conditional distribution of the observed characteristics of claimants with earnings around $T^{\min }$ is not smooth. Given this situation, we have to interpret the estimated parameters derived from the bottom kink sample carefully, acknowledging that there is a likely upward bias in the RKD estimate of the elasticity of the duration of joblessness with respect to UI benefits, driven by the non-smoothness in the observed determinants of joblessness durations.

For the top kink sample (Panel B) there is less evidence of non-smoothness, though we note that the bias-corrected local linear model and the local quadratic models point to a possible negative kink in the predicted log time to next job. Taken together with the relatively smooth patterns in Figure 8 and Appendix Figures 3a-3d, however, we believe that the assumptions for a valid regression kink are plausibly satisfied around the top kink. 


\subsubsection{Fuzzy RKD Estimates and Comparison of Alternative Estimators}

As a final step in our empirical analysis we present fuzzy RKD estimates of the elasticity of the duration of joblessness with respect to the level of UI benefits and evaluate the performance of alternative estimators. As noted in subsections 2.2.2 and 4.2, the fuzzy RK estimand in the bottom kink sample identifies the behavioral response for claimants whose baseline earnings are close to $T^{\min }$ and who follow the benefit schedule intended for single claimants. In the top kink sample, the fuzzy RK estimand identifies the elasticity for claimants close to $T^{\max }$ who follow any of the benefit schedules seen in Figure 1. Therefore, applying a regression kink approach to the two samples allows us to estimate the elasticity of joblessness with respect to UI benefit generosity for two very different subpopulations. ${ }^{31}$ Table 4 presents estimated elasticities for the local linear and quadratic estimators under the FG bandwidth. We show both the conventional estimates (columns 2 and 5) and the bias-corrected estimates (columns 3 and 6) with robust confidence intervals that take account of the sampling variability of the bias corrections. Without bias correction, the local linear models yield estimated elasticities of 1.4 and 2.0 for the bottom kink and top kink samples, respectively. In both cases, the corresponding bias-corrected estimates are larger in magnitude, as are the estimates from the local quadratic models, suggesting that if anything, the uncorrected local linear estimates are "conservative".

In Table 5, we present elasticities along with first-stage estimates for four alternative bandwidth selection procedures. The alternatives we consider are: default CCT, CCT with no regularization, Fuzzy CCT and Fuzzy IK. (Expressions for the latter two bandwidths are given in subsection B.2 of the Supplemental Appendix). For each bandwidth selector we present the value of the main and pilot bandwidth (the pilot bandwidth is for bias estimation in constructing the CCT confidence interval), the uncorrected and biascorrected first stage kink estimates, and the uncorrected and bias-corrected structural elasticities. The first stage kink estimates are generally similar to the estimates presented in Panel A of Table 2a, but they are not identical because the bandwidths in Table 5 are selected to be optimal for the numerator of the RKD estimand, or for the estimand itself (in the case of the fuzzy CCT and fuzzy IK procedures) rather than for the first stage equation.

The pattern of estimates in Table 5 point to three main conclusions. First, as we noted in the discussion of Table 2a, many of the bandwidth selectors choose relatively small bandwidths that lead to relatively im-

\footnotetext{
${ }^{31}$ It may be tempting to apply a sharp RK design to only the observations that lie on the UI schedule. However, this approach does not in general identify an interpretable treatment effect, just as an analysis of the subset of "compliers" in a randomized experiment is likely to be highly problematic.
} 
precise first-stage and structural coefficient estimates. A second observation is that, as in Table 2a, the local quadratic estimators are generally quite noisy. Third, the bias-corrected estimates from the local linear models are typically not too different from the uncorrected estimates, but the added imprecision associated with uncertainty about the magnitude of the bias correction factor is large, leading to relatively wide confidence intervals for the bias corrected estimates.

Given the wide range of estimates in Tables 4 and 5, which specification should we pick? The line of argument advanced in Calonico et al. (Forthcoming) suggests that one might prefer the bias-corrected estimates from local quadratic models using the CCT bandwidth selector with regularization. In our application, these estimates are imprecise. For the bottom kink sample this choice suggests the elasticity of joblessness with respect to UI benefits is 19.0, whereas for the top kink sample the estimate is wrong-signed and equal to -6.9. However, given the wide confidence intervals both estimates are essentially uninformative. At the opposite extreme, the uncorrected estimates from local linear specifications using the FG bandwidth selector (in Table 4) are relatively precisely determined and point to behavioral elasticities in the range of 1 to 2 .

To gain additional insight, we decided to conduct a series of Monte Carlo simulations based on DGP's that closely resemble our actual samples. Because we are interested in the power of the candidate estimators, we impose the first-stage kink parameter and the elasticity parameter in constructing the DGP's. For the bottom kink sample, we impose the first-stage kink as $\tau_{B}=2.3 \times 10^{-5}$ and the elasticity $\tau_{F R K D}=1.3$, which implies a reduced-form kink of $\tau_{Y}=\tau_{B} \cdot \tau_{F R K D}=3.0 \times 10^{-5}$. For the top kink sample, we set $\tau_{B}=-1.4 \times 10^{-5}$ and $\tau_{F R K D}=2.0$ with an implied $\tau_{Y}=-2.8 \times 10^{-5}$. We then specify the DGP's for $E[B \mid V]$ and $E[Y \mid V]$ as separate quintics on each side of the threshold, where the parameters of the quintics are estimated by regressing, respectively, $B-\tau_{B} \cdot D \cdot V$ and $Y-\tau_{Y} \cdot D \cdot V$ on the polynomial terms $V^{j}$ and $D \cdot V^{k}$ where $D=1_{[V \geqslant 0]}, j=0,1 \ldots, 5$ and $k=2, \ldots, 5$. For our simulation, we sample $V$ from its empirical distribution and the errors $\left(\varepsilon_{B}, \varepsilon_{Y}\right)$ jointly from the residuals from the quintic regressions, and construct $B=E[B \mid V]+\varepsilon_{B}$ and $Y=E[Y \mid V]+\varepsilon_{Y}$. We draw 1,000 repeated samples in our Monte Carlo exercise.

Tables $6 \mathrm{a}$ and $6 \mathrm{~b}$ summarize the performance of the alternative estimators for the simulated bottom kink and top kink samples, respectively. The two polynomial orders (linear and quadratic), two bias-correction choices (uncorrected and bias-corrected) and six bandwidth selection procedures (default CCT, CCT with no regularization, Fuzzy CCT, Fuzzy IK, FG, and Global) give rise to 24 candidate estimators in total. For each estimator, we report the associated bandwidth(s) and its performance in estimating the first-stage and the elasticity parameters. 
The two main criteria for evaluating estimator performance are 1) the root mean squared error (RMSE) of the elasticity estimator; and 2) how often its confidence interval covers the true parameter $\tau_{F R K D}$. In columns (6) and (7) we report two measures of RMSE. Column (6) shows the raw RMSE as a ratio of the imposed $\tau_{F R K D}$. Because we worry that outliers may drive the numbers in column (6), we report in column (7) a "trimmed" RMSE ratio by first discarding 5\% of the simulation sample with the greatest deviation between $\hat{\tau}_{F R K D}$ and $\tau_{F R K D}$. In column (8), we report the coverage rates of the confidence interval.

As seen from Table 6, none of the estimators consistently achieves the lowest RMSE and delivers the correct coverage rate, but the conventional local linear estimator with the FG bandwidth (LLFG for short) appears to be a reasonable choice. In the bottom kink sample, this estimator and the global conventional linear estimator outperform all other candidates, and the two are very similar in terms of their RMSE's and coverage rates (96\% and 94\% respectively). In the top kink sample, the LLFG estimator has the smallest RMSE, although the coverage rate of the corresponding confidence interval is a lower $82 \%$. The lower coverage rate is indicative of the bias of the LLFG estimator, but the bias is quite small in magnitude (0.11) as compared to the imposed elasticity (2.0). A viable alternative for the top kink sample is the local linear estimator with the fuzzy IK bandwidth (LLIK for short): it has a moderately higher RMSE, but the corresponding confidence interval has a better coverage rate at 94\% than LLFG. As shown in Table 5, using LLIK also points to a statistically significant high elasticity, which is consistent with the LLFG result.

Analogously to Table 2b, we also break down the (trimmed) MSE and report the bias, squared bias and variance of each of the estimators in columns (9)-(11) of Table 6. Bias correction appears to increase both the squared bias and the variance for all estimators in the bottom kink simulations and for 8 out of 12 estimators in the top kink simulations. In 23 out of the 24 cases, bias correction increases the (trimmed) RMSE, with the only exception being the global quadratic estimator for the top kink.

In a parallel simulation study, we assess the performance of the estimators in DGP's where we impose the true elasticity $\tau_{F R K D}$ to be zero: the results are presented in Appendix Table 2. In terms of RMSE, the FG bandwidth still outperforms the four local alternatives, and within each bandwidth choice, the conventional linear estimator dominates the other three candidates. In terms of the coverage rate of the corresponding $95 \%$ confidence interval, however, LLFG no longer performs well: the coverage rate is $2 \%$ for the bottom kink simulation and $20 \%$ for the top. Moreover, there does not appear to be an attractive alternative estimator: those with a coverage rate above $90 \%$ have a variance at least 30 times higher than LLFG in the top kink sample and at least 80 times higher in the bottom kink sample. 
The poor coverage rates of the LLFG confidence interval in Appendix Table 2 raise the concern that the structural elasticities arising from applying this procedure to our actual data may be misleading. However, the value of the bias-corrected LLFG estimator is higher than the conventional LLFG estimator in our actual data (1.95 versus 1.37 for the bottom kink; 3.67 versus 2.04 for the top kink), and this empirical regularity is matched by the DGP's underlying Table 6 but not by the DGP's underlying Appendix Table 2. Therefore, we believe that the simulation DGP's underlying Table 6, as opposed to those underlying Appendix Table 2, are better approximations to the actual data.

In another exercise, we provide further evidence that the local linear estimator should be preferred to the local quadratic by directly estimating the AMSE's for the local linear and quadratic estimators, per Card et al. (2014). As shown in Appendix Table 3, using the default CCT bandwidth selection procedure, the AMSE for the local quadratic model is at least an order of magnitude larger than the AMSE for the local linear model. ${ }^{32}$ For the other bandwidth choices, the linear AMSE is also much smaller (at least $68 \%$ smaller) than the quadratic AMSE, and we omit them from Appendix Table 3 for ease of exposition.

As a final robustness check, we investigate how sensitive the elasticity estimates are with respect to the choice of bandwidth. Figures 9 and 10 plot the elasticity estimates for the bottom and top kink samples associated with a range of potential bandwidths. Ruppert (1997) argues that one can use the relationship between the point estimates and the bandwidth choice as an indicator of potential bias, with stability in the estimate indicating the absence of significant bias. Figure 9 shows that the estimated elasticity of time to next job with respect to UI benefits around the bottom kink is relatively stable at close to 1.4 for a very wide range of bandwidths. Figure 10 shows that the estimated elasticity around the top kink is a little more sensitive to bandwidth choice, with a larger estimate (between 2 and 3) for lower bandwidths, but an elasticity of 2 or less for bandwidths above $€ 5,000$.

Overall, we conclude that the conventional local linear estimator with the FG bandwidth does reasonably well for our empirical application. The corresponding estimates lead us to two main findings. First, for job losers from the upper part of the earnings distribution (around $T^{\max }$ ), the elasticity of the time to next job with respect to UI benefits is around 2. Our confidence in this estimate is strengthened by the fact that tests for the validity of an RK design around the top kink show little evidence that the design is compromised by sorting.

\footnotetext{
${ }^{32}$ Note that the estimated bias component for the local quadratic estimator is substantially larger than its local linear counterpart, even though the former shrinks to zero at a faster rate.
} 
A second, more tentative conclusion is that the corresponding elasticity for job losers in the lower part of the earnings distribution (around $T^{\min }$ ) is of a smaller magnitude - perhaps closer to 1 . Our cautious assessment stems from the fact that the tests for the validity of an RKD approach show that the conditional distributions of observed worker characteristics change slopes around $T^{\min }$. These changes are not associated with any discernible "bunching" at the kink point in the benefit schedule, but they are large enough to cause a 30\% upward bias in the estimated jobless elasticity.

How do our estimated benefit elasticities compare to those in the existing literature? Appendix Table 4 contains a brief summary of the existing literature, drawing on the survey by Krueger and Meyer (2002) for the earlier U.S.-based literature, all of which use administrative records on unemployment insurance claims and estimate the effect of UI benefits on the duration of the initial spell of insured unemployment. These studies point to a benefit elasticity in the range of 0.3 to $0.8 .^{33}$ A recent study by Landais (Forthcoming) applies a regression kink design to some of the same data used in these earlier studies and obtains estimates of the elasticity of the initial UI benefit spell that range from 0.20 to 0.70 . Another recent study by Chetty (2010) uses retrospective interview data from the Survey of Income and Program Participation and obtains an average benefit elasticity of about 0.5 . Taken together, these U.S. studies suggest a benchmark of around 0.5 for the elasticity of initial UI claim duration on the UI benefit. Most of the European studies included in Appendix Table 4 estimate the effect of benefits on the time to first exit from the UI system and obtain benefit elasticities that are similar to the U.S. studies. An exception is Carling et al. (2011), who study the effect of a reduction in the benefit replacement rate in Sweden in 1996 on the exit rate from unemployment recipiency to employment. Their estimate of the elasticity of time to next job with respect to the benefit level is 1.6, which is not far from our point estimate of 2.0 for the top kink sample.

In Card et al. (2012), we argued that the high elasticity we find may be a consequence of using time to next job as an outcome measure as opposed to the insured unemployment duration typically used in the literature. Our findings underscore the potential value in being able to measure time to next job in assessing the incentive effects of the UI system.

\footnotetext{
${ }^{33}$ Krueger and Meyer attribute an estimated elasticity of 1.0 to Solon (1985) who studies the effect of making UI benefits taxable on the unemployment duration of high-earning claimants. He finds that the introduction of taxation caused a $22 \%$ reduction in the average duration of initial UI claims by higher-earning claimants (with no effect on low-earners). Assuming an average tax rate of $30 \%$, this implies an elasticity of 0.73 , which is our preferred interpretation of Solon's results.
} 


\section{Conclusion}

In many institutional settings, a key policy variable (like unemployment benefits or public pensions) is set by a deterministic formula that depends on an endogenous assignment variable (like previous earnings). Conventional approaches to causal inference, which rely on the existence of an instrumental variable that is correlated with the covariate of interest but independent of underlying errors in the outcome, will not work in these settings. When the policy function is continuous but kinked (i.e., non-differentiable) at a known threshold, a regression kink design provides a potential way forward (Guryan (2001); Nielsen et al. (2010); Simonsen et al. (Forthcoming)). The sharp RKD estimand is simply the ratio of the estimated kink in the relationship between the assignment variable and the outcome of interest at the threshold point, divided by the corresponding kink in the policy function. In settings where there is incomplete compliance with the policy rule (or measurement error in the actual assignment variable), a "fuzzy RKD" replaces the denominator of the RKD estimand with the estimated kink in the relationship between the assignment variable and the policy variable.

In this paper, we provide sufficient conditions for a sharp and fuzzy RKD to identify interpretable causal effects in a general nonseparable model (e.g., Blundell and Powell (2003)). The key assumption is that the conditional density of the assignment variable, given the unobserved error in the outcome, is continuously differentiable at the kink point. This smooth density condition rules out situations where the value of the assignment variable can be precisely manipulated, while allowing the assignment variable to be correlated with the latent errors in the outcome. Thus, extreme forms of "bunching" predicted by certain behavioral models (e.g., Saez (2010)) violate the smooth density condition, whereas similar models with errors in optimization (e.g., Chetty (2010)) are potentially consistent with an RKD approach. In addition to yielding a testable smoothness prediction for the observed distribution of the assignment variable, we show that the smooth density condition also implies that the conditional distributions of any predetermined covariates will be smooth functions of the assignment variable at the kink point. These two predictions are very similar in spirit to the predictions for the density of the assignment variable and the distribution of predetermined covariates in a regression discontinuity design (Lee (2008)).

We also provide a precise characterization of the treatment effects identified by a sharp or fuzzy RKD. The sharp RKD identifies a weighted average of marginal effects, where the weight for a given unit reflects the relative probability of having a value of the assignment variable close to the kink point. Under an addi- 
tional monotonicity assumption, we show that the fuzzy RKD identifies a slightly more complex weighted average of marginal effects, where the weight also incorporates the relative size of the kink induced in the actual value of the policy variable for that unit.

We illustrate the use of a fuzzy RKD approach by studying the effect of unemployment benefits on the duration of joblessness in Austria, where the benefit schedule has kinks at the minimum and maximum benefit levels. We present a variety of simple graphical evidence showing that these kinks induce kinks in the duration of total joblessness between the end of the previous job and the start of the next job. We also present a variety of tests of the smooth density assumption around the thresholds for the minimum and maximum benefit amounts. We present alternative estimates of the behavioral effect of higher benefits on the duration of joblessness and evaluate the empirical performance of the alternative estimators using Monte Carlo simulation. Our preferred estimates point to elasticities that are higher than most of the previous studies in the United States and Europe. 


\section{References}

Altonji, Joseph G. and Rosa L. Matzkin, "Cross Section and Panel Data Estimators for Nonseparable Models with Endogenous Regressors,” Econometrica, 2005, 73 (4), 1053-1102.

Ando, Michihito, “How Much Should We Trust Regression-Kink-Design Estimates?," January 2014.

Arraz, Jose M., Fernando Munoz-Bullon, and Juan Muro, "Do Unemployment Benefit Legislative Changes Affect Job Finding?," Working Paper, Universidad de Alcalä 2008.

Baily, Martin N., "Some Aspects of Optimal Unemployment Insurance," Journal of Public Economics, 1978, 10 (3), 379-402.

Blundell, Richard and James L. Powell, "Endogeneity in Nonparametric and Semiparametric Regression Models," in Mathias Dewatripont, Lars Peter Hansen, and Stephen J. Turnovsky, eds., Advances in economics and econometrics theory and applications : Eighth World Congress., Vol. II of Econometric Society monographs no. 36, Cambridge: Cambridge University Press, 2003, pp. 312-357.

Calonico, Sebastian, Matias D. Cattaneo, and Rocio Titiunik, "Optimal Data-driven Regression Discontinuity Plots," October 2014.

_ , _ , and _ , "rdrobust: An R Package for Robust Inference in Regression-Discontinuity Designs," Technical Report, University of Michigan 2014.

_, , , and _ , "Robust Nonparametric Confidence Intervals for Regression-Discontinuity Designs," Econometrica, Forthcoming.

${ }_{-},{ }_{-}$, and _ , "Robust Data-Driven Inference in the Regression-Discontinuity Design," Stata Journal, in press.

Card, David, David S. Lee, Zhuan Pei, and Andrea Weber, "Nonlinear Policy Rules and the Identification and Estimation of Causal Effects in a Generalized Regression Kink Design," NBER Working Paper 18564 November 2012.

_, _, , and _ , "Local Polynomial Order in Regression Discontinuity Designs,” October 2014.

Carling, Kenneth, Bertil Holmlund, and Altin Vejsiu, "Do Benefit Cuts Boost Job Finding? Swedish Evidence from the 1990s," Economic Journal, 2011, 111 (474), 766-790.

Cheng, Ming-Yen, Jianqing Fan, and J. S. Marron, "On automatic boundary corrections," The Annals of Statistics, 08 1997, 25 (4), 1691-1708.

Chesher, Andrew, "Identification in Nonseparable Models," Econometrica, 2003, 71 (5), 1405-1441.

Chetty, Raj, "Moral Hazard versus Liquidity and Optimal Unemployment Insurance," Journal of Political Economy, 2010, 116 (2), 173-234.

_, "Bounds on Elasticities with Optimization Frictions: A Synthesis of Micro and Macro Evidence on Labor Supply," Econometrica, 2012, 80 (3), 969-1018.

Christensen, Bent Jesper, Rasmus Lenz, Dale T. Mortensen, George R. Neumann, and Axel Werwatz, "On the Job Search and the Wage Distribution," Journal of Labor Economics, 2005, 23 (1), 181-221. 
Classen, Kathleen P., "The Effect of Unemployment Insurance on the Duration of Unemployment and Subsequent Earnings," Industrial and Labor Relations Review, 1977, 30(8), 438-444.

_ , "Unemployment Insurance and Job Search," in S. Lippman and J. McCall, eds., Studies in the Economics of Job Search, North-Holland, 1979, pp. 191-219.

Dahlberg, Matz, Eva Mork, Jorn Rattso, and Hanna Agren, "Using a Discontinuous Grant Rule to Identify the Effect of Grants on Local Taxes and Spending," Journal of Public Economics, 2008, 92 (12), $2320-2335$.

Del Bono, Emilia and Andrea Weber, "Do Wages Compensate for Anticipated Working Time Restrictions? Evidence from Seasonal Employment in Austria," Journal of Labor Economics, 2008, 26 (1), 181-221.

DiNardo, John E. and David S. Lee, "Program Evaluation and Research Designs," in Orley Ashenfelter and David Card, eds., Handbook of Labor Economics, Vol. 4A, Elsevier, 2011.

Dong, Yingying, "Regression Discontinuity without the Discontinuity," Technical Report, University of California Irvine 2013.

- and Arthur Lewbel, "Identifying the Effect of Changing the Policy Threshold in Regression Discontinuity Models,” 2014.

Fan, Jianqing and Irene Gijbels, Local Polynomial Modelling and Its Applications, Chapman and Hall, 1996.

Florens, Jean-Pierre, James J. Heckman, Costas Meghir, and Edward J. Vytlacil, "Identification of Treatment Effects Using Control Functions in Models With Continuous, Endogenous Treatment and Heterogeneous Effects," Econometrica, 2008, 76 (5), 1191-1206.

Ganong, Peter and Simon Jäger, "A Permutation Test and Estimation Alternatives for the Regression Kink Design," June 2014.

Guryan, Jonathan, "Does Money Matter? Regression-Discontinuity Estimates from Education Finance Reform in Massachusetts," Working Paper 8269, National Bureau of Economic Research 2001.

Hahn, Jinyong, Petra Todd, and Wilbert Van der Klaauw, "Identification and Estimation of Treatment Effects with a Regression-Discontinuity Design,” Econometrica, 2001, 69 (1), 201-209.

Heckman, James J. and Edward J. Vytlacil, "Structural Equations, Treatment Effects, and Econometric Policy Evaluation,” Econometrica, 2005, 73 (3), 669-738.

Imbens, Guido W. and Joshua D. Angrist, "Identification and Estimation of Local Average Treatment Effects," Econometrica, 1994, 62 (2), 467-475.

_ and Karthik Kalyanaraman, "Optimal Bandwidth Choice for the Regression Discontinuity Estimator.," Review of Economic Studies, 2012, 79 (3), 933 - 959.

- and Thomas Lemieux, "Regression Discontinuity Designs: A Guide to Practice," Journal of Econometrics, February 2008, 142 (2), 615-635.

_ and Whitney K. Newey, "Identification and Estimation of Triangular Simultaneous Equations Models Without Additivity,” Econometrica, 2009, 77 (5), 1481-1512. 
Inoue, Atsushi and Gary Solon, "Two-Sample Instrumental Variables Estimators," The Review of Economics and Statistics, August 2010, 92 (3), 557-561.

Kapteyn, Arie and Jelmer Y. Ypma, "Measurement Error and Misclassification: A Comparison of Survey and Administrative Data," Journal of Labor Economics, 2007, 25 (3), 513-551.

Katz, Lawrence H. and Bruce D. Meyer, "The Impact of the Potential Duration of Unemployment Benefits on the Duration of Unemployment," Journal of Public Economics, 1990, 41 (1), 45-72.

Kroft, Kory, "Takeup, Social Multipliers and Optimal Social Insurance," Journal of Public Economics, 2008, 92 (3-4), 722-737.

Krueger, Alan B. and Bruce D. Meyer, "Labor Supply Effects of Social Insurance," in Alan J. Auerbach and Martin S Feldstein, eds., Handbook of Public Economics, Amsterdam and New York: Elsevier, 2002, pp. 2327-2392.

Lalive, Rafael, Jan C. Van Ours, and Josef Zweimüller, "How Changes in Financial Incentives Affect the Duration of Unemployment," Review of Economic Studies, 2006, 73 (4), 1009-1038.

Landais, Camille, "Assessing the Welfare Effects of Unemployment Benefts Using the Regression Kink Design,” American Economic Journal: Economic Policy, Forthcoming.

Lee, David S., "Randomized Experiments from Non-random Selection in U.S. House Elections," Journal of Econometrics, February 2008, 142 (2), 675-697.

_ and Thomas Lemieux, "Regression Discontinuity Designs in Economics," Journal of Economic Literature, 2010, 48 (2), 281-355.

Lewbel, Arthur, "Semiparametric Latent Variable Model Estimation with Endogenous or Mismeasured Regressors," Econometrica, 1998, 66 (1), 105-121.

_, "Semiparametric Qualitative Response Model Estimation with Unknown Heteroscedasticity or Instrumental Variables," Journal of Econometrics, 2000, 97, 145-177.

Lindsay, Bruce G. and Annie Qu, "Inference Functions and Quadratic Score Tests," Statistical Science, 2003, 18 (3), 394-410.

McCrary, Justin, "Manipulation of the Running Variable in the Regression Discontinuity Design: A Density Test," Journal of Econometrics, 2008, 142 (2), 698-714.

Meyer, Bruce D., "Unemployment Insurance and Unemployment Spells," Econometrica, 1990, 58 (4), 757-782.

- and Wallace K. C. Mok, "Quasi-Experimental Evidence on the Effects of Unemployment Insurance from New York State,” NBER Working Paper 128652007.

Moffitt, Robert, “Unemployment Insurance and the Distribution of Unemployment Spells," Journal of Econometrics, 1985, 28 (1), 85-101.

Nielsen, Helena Skyt, Torben Sørensen, and Christopher R. Taber, "Estimating the Effect of Student Aid on College Enrollment: Evidence from a Government Grant Policy Reform," American Economic Journal: Economic Policy, 2010, 2 (2), 185-215. 
Roed, Knut and Tao Zhang, "Does Unemployment Compensation Affect Unemployment Duration?," Economic Journal, 2003, 113 (484), 190-206.

Roussas, George G., An Introduction to Measure-theoretic Probability, Academic Press, 2004.

Ruppert, David, "Empirical-Bias Bandwidths for Local Polynomial Nonparametric Regression and Density Estimation,” Journal of the American Statistical Association, 1997, 92 (439), pp. 1049-1062.

Saez, Emmanuel, "Do Taxpayers Bunch at Kink Points?," American Economic Journal: Economic Policy, 2010, 2 (3), 180-212.

Simonsen, Marianne, Lars Skipper, and Niels Skipper, "Price Sensitivity of Demand for Prescription Drugs: Exploiting a Regression Kink Design,” Journal of Applied Econometrics, Forthcoming.

Solon, Gary, "Work Incentive Effects of Taxing Unemployment Benefits," Econometrica, 1985, 53 (2), 295-306.

Thistlethwaite, Donald L. and Donald T. Campbell, "Regression-Discontinuity Analysis: An Alternative to the Ex-Post Facto Experiment," Journal of Educational Psychology, 1960, 51 (6), 309-317.

Turner, Lesley J., "The Road to Pell is Paved with Good Intentions: The Economic Incidence of Federal Student Grant Aid," Technical Report, University of Maryland 2013.

Vytlacil, Edward J., "Independence, Monotonicity, and Latent Index Models: An Equivalence Result," Econometrica, 2002, 70 (1), 331-341.

Welch, Finis, "What have we learned from empirical studies of unemployment insurance?," Industrial and Labor Relations Review, July 1977, 30 (4), 451-461.

Zorich, Vladimir A., Mathematical Analysis II, Springer, 2004.

Zweimüller, Josef, Rudolf Winter-Ebmer, Rafael Lalive, Andreas Kuhn, Jean-Philippe Wuellrich, Oliver Ruf, and Simon Büchi, "Austrian Social Security Database, University of Zurich," Working Paper iewwp410, Institute for Empirical Research in Economics 2009. 
Figure 1: UI Benefits in 2004

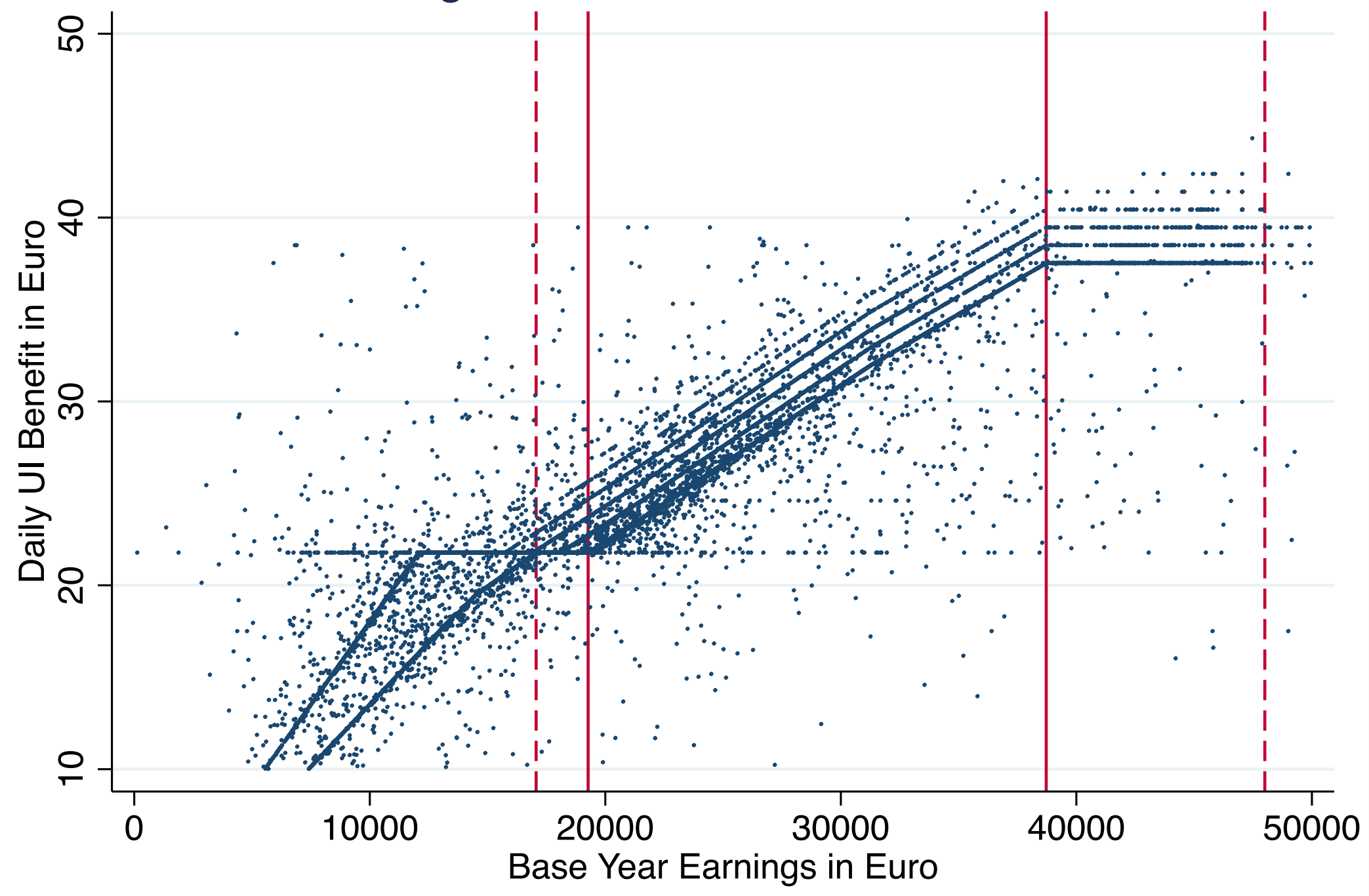



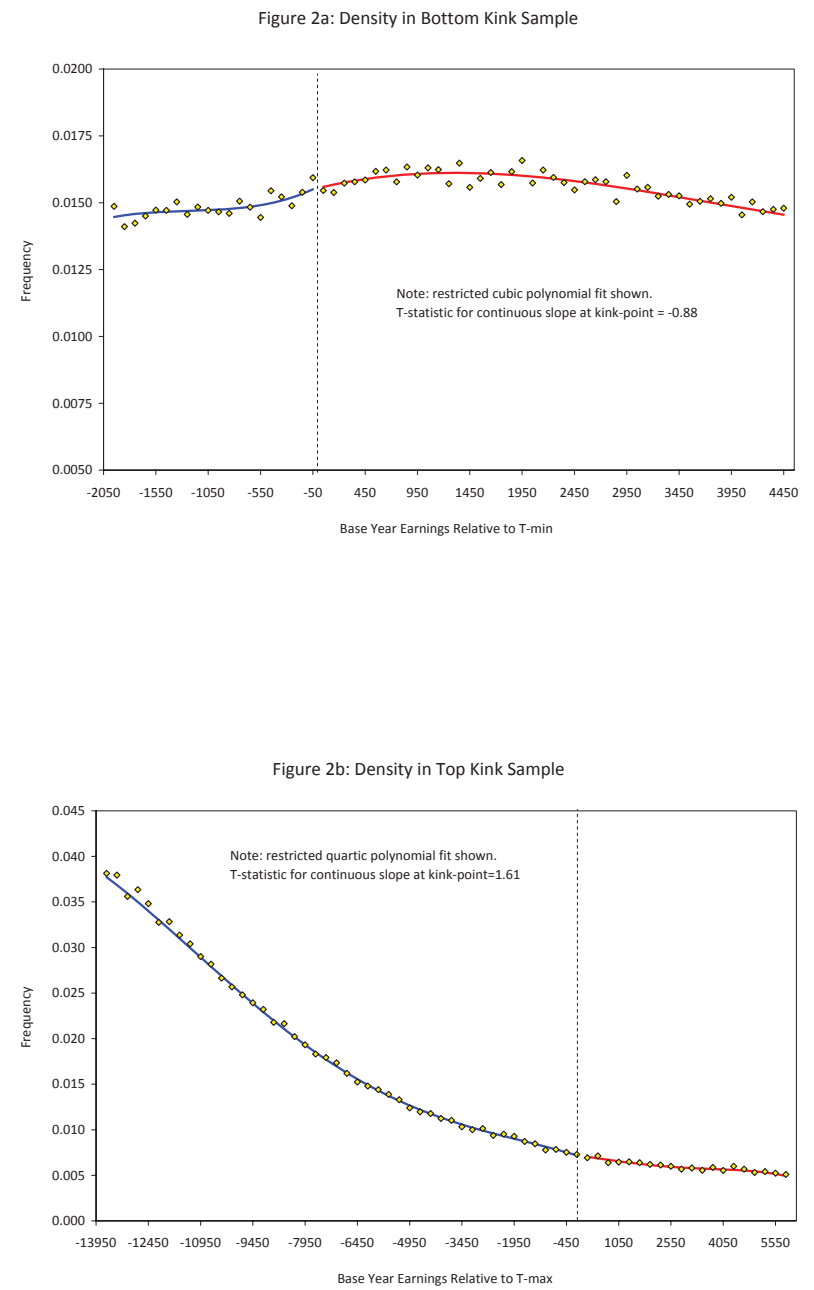
Figure 3: Daily UI Benefits Bottom Kink Sample

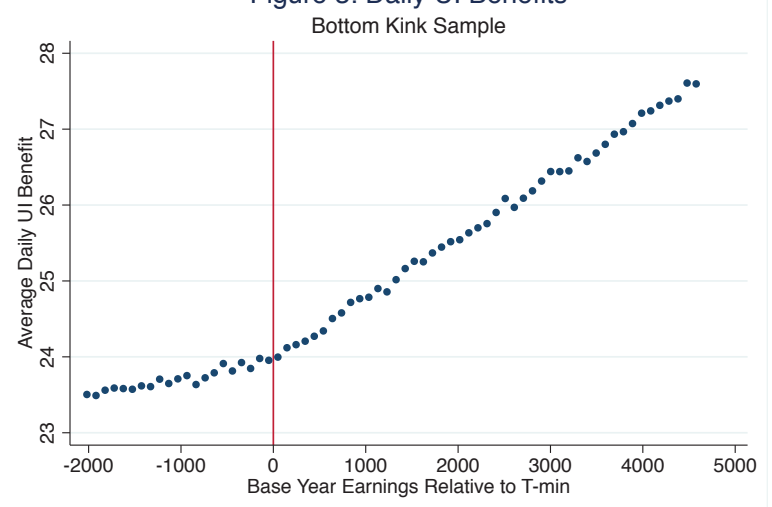

Figure 4: Daily UI Benefits

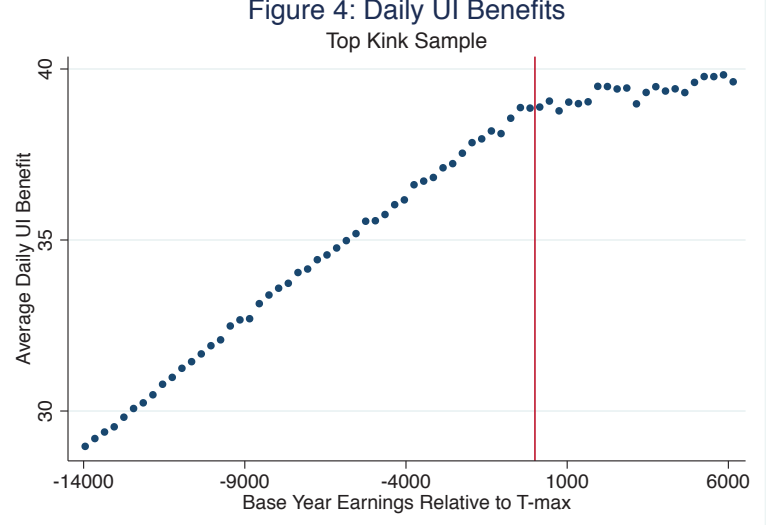


Figure 5: Log Time to Next Job

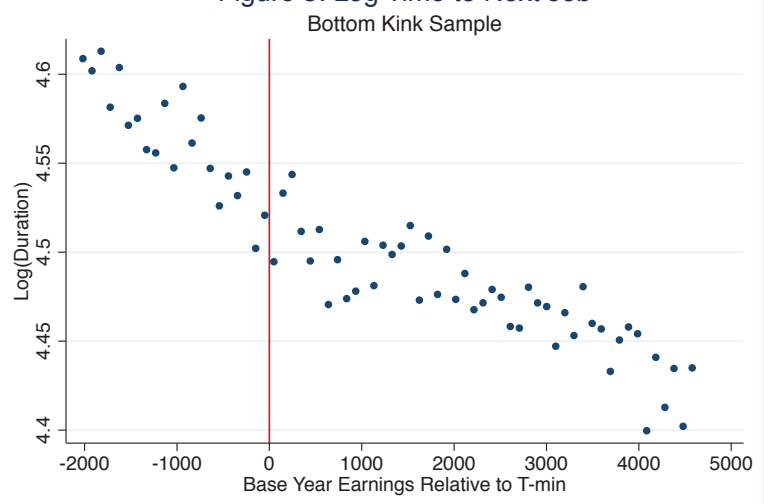

Figure 6: Log Time to Next Job

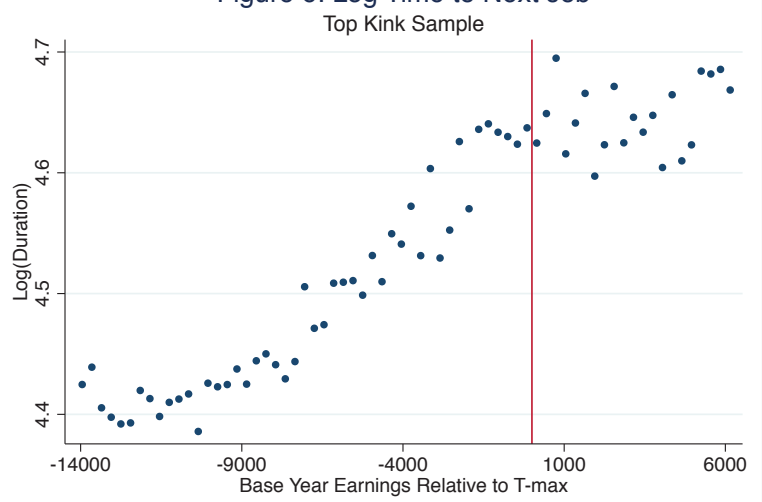


Figure 7: Predicted Time to Next Job

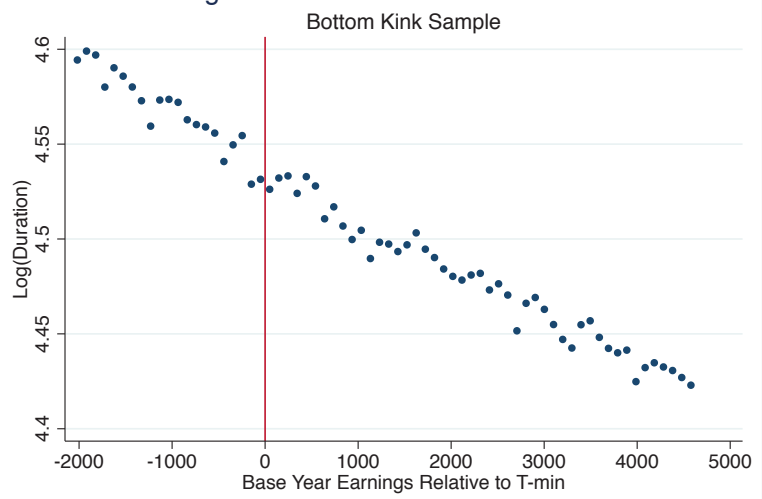

Figure 8: Predicted Time to Next Job

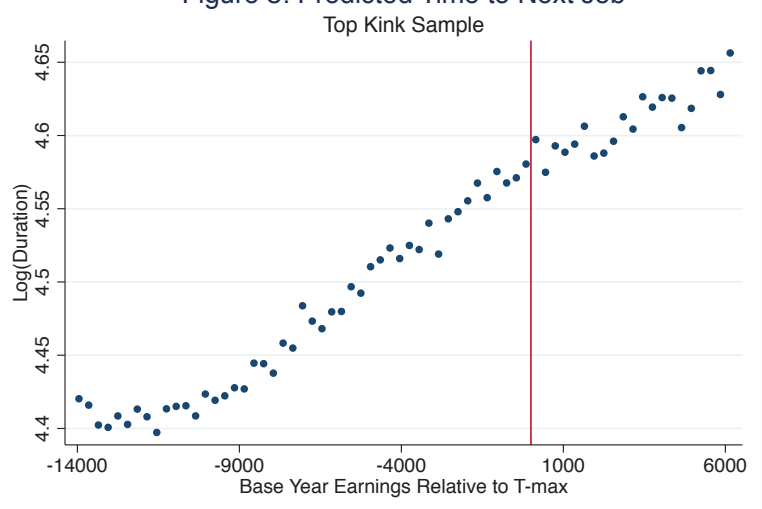


Figure 9: Fuzzy RKD estimation with varying bandwidth

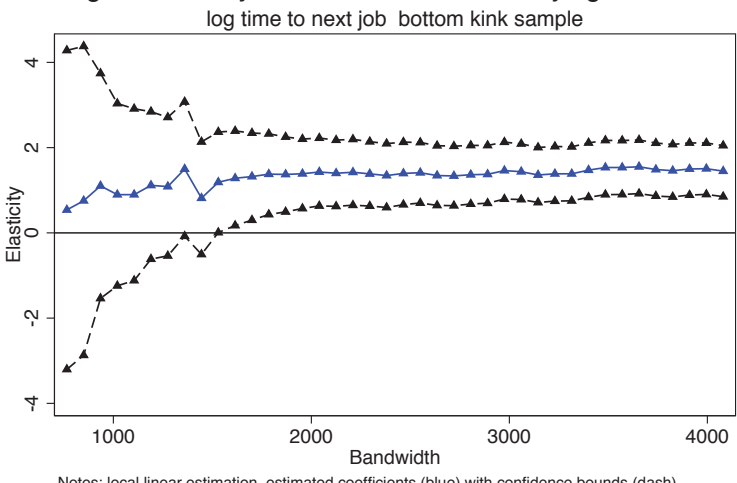

Notes: local linear estimation, estimated coefficients (blue) with confidence bounds (dash)

Figure 10: Fuzzy RKD estimation with varying bandwidth log time to next job top kink sample

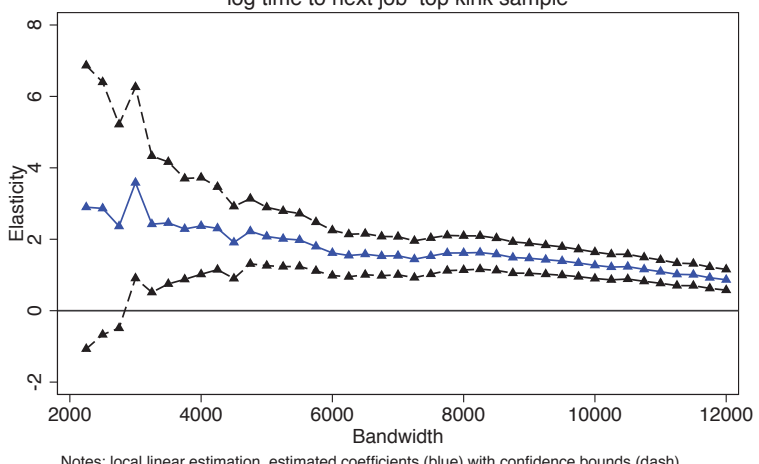


Table 1: Summary Statistics for Bottom and Top Kink Samples of UI Claimants

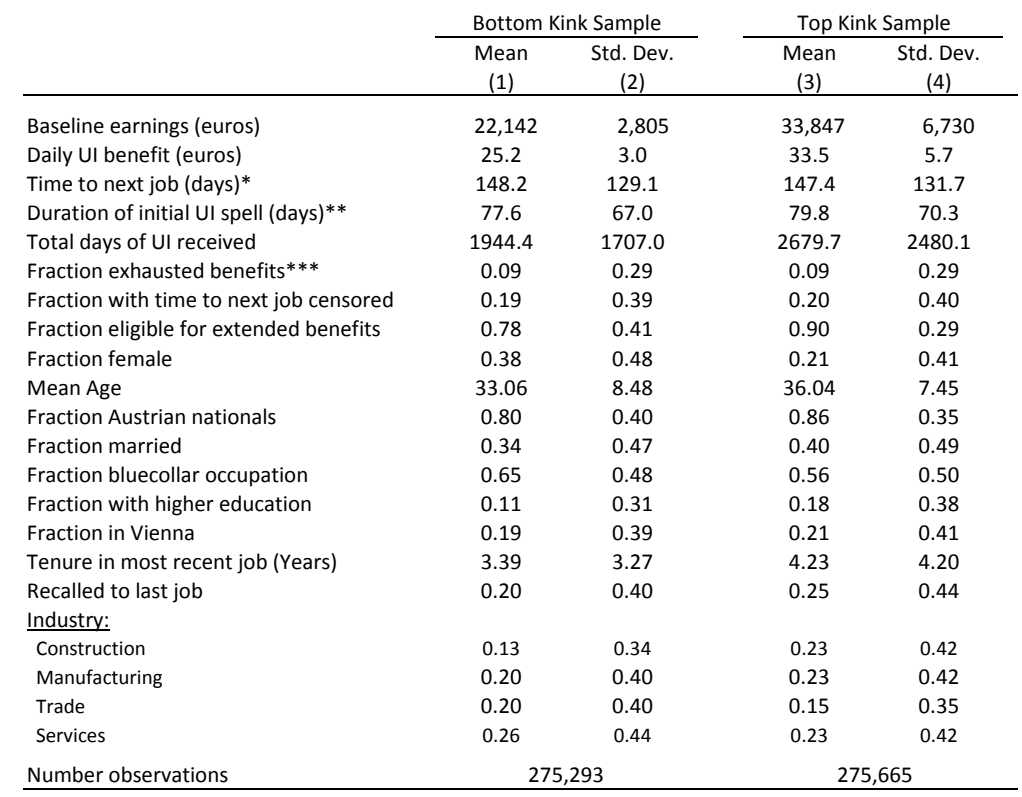

Notes: sample contains UI claimants under the age of 50 with claims in 2001-2012, who had at least 1 year of tenure on their previous job, began their claim within 4 weeks of losing their past job, and had a valid UI claim record and non-missing earnings in the base period prior to the claim. Observations in the bottom kink sample have base period earnings in a range around the bottom kink in the UI benefit schedule; observations in the top kink sample have base period earnings in a range around top kink. See text.

* Time to next job is censored at 365 days.

** Claim duration is censored at 39 weeks (maximum entitlement).

*** Indicator equals 1 if claim duration = maximum entitlement. 
Table 2a: First Stage and Reduced Form Estimated Kinks

\begin{tabular}{|c|c|c|c|c|}
\hline & \multicolumn{2}{|c|}{ Bottom Kink } & \multicolumn{2}{|c|}{ Top Kink } \\
\hline & $\begin{array}{l}\text { Local Linear } \\
\text { (1) }\end{array}$ & $\begin{array}{l}\text { Local Quad. } \\
\text { (2) }\end{array}$ & $\begin{array}{l}\text { Local Linear } \\
\text { (3) }\end{array}$ & $\begin{array}{l}\text { Local Quad. } \\
\text { (4) }\end{array}$ \\
\hline \multicolumn{5}{|c|}{ A. First Stage Model (Dependent variable $=\log$ daily benefit) } \\
\hline \multicolumn{5}{|c|}{$\begin{array}{l}\text { Default CCT (with regularization) } \\
\end{array}$} \\
\hline $\begin{array}{l}\text { Estimated Kink } \\
\text { (conventional std error) }\end{array}$ & $\begin{array}{c}1.7 \\
(1.0)\end{array}$ & $\begin{array}{c}2.1 \\
(1.6)\end{array}$ & $\begin{array}{l}-1.5 \\
(0.7)\end{array}$ & $\begin{array}{l}-1.1 \\
(2.2)\end{array}$ \\
\hline $\begin{array}{l}\text { Estimated Kink - Bias Corrected } \\
\text { [robust confidence interval] }\end{array}$ & $\begin{array}{c}1.2 \\
{[-1.6,3.9]}\end{array}$ & $\begin{array}{c}1.2 \\
{[-2.7,5.1]}\end{array}$ & $\begin{array}{c}-1.6 \\
{[-3.6,0.4]}\end{array}$ & $\begin{array}{c}-2.3 \\
{[-7.6,3.1]}\end{array}$ \\
\hline \multicolumn{5}{|l|}{ CCT with no regularization } \\
\hline Main (Pilot) Bandwidth & $1,257(1,677)$ & $1,220(1,646)$ & $2,913(3,813)$ & $2,128(3,031)$ \\
\hline $\begin{array}{l}\text { Estimated Kink } \\
\text { (conventional std error) }\end{array}$ & $\begin{array}{c}2.3 \\
(0.2)\end{array}$ & $\begin{array}{l}1.8 \\
(0.9)\end{array}$ & $\begin{array}{l}-1.2 \\
(0.2)\end{array}$ & $\begin{array}{l}-3.6 \\
(1.4)\end{array}$ \\
\hline $\begin{array}{l}\text { Estimated Kink - Bias Corrected } \\
\text { [robust confidence interval] }\end{array}$ & $\begin{array}{c}2.1 \\
{[1.0,3.1]}\end{array}$ & $\begin{array}{c}1.1 \\
{[-1.6,3.7]}\end{array}$ & $\begin{array}{c}-0.9 \\
{[-2.0,0.2]}\end{array}$ & $\begin{array}{c}-4.7 \\
{[-8.7,-0.7]}\end{array}$ \\
\hline \multicolumn{5}{|l|}{ FG } \\
\hline Main (Pilot) Bandwidth & $2,466(2,380)$ & $2,628(4,146)$ & $11,603(6,343)$ & $7,003(13,081)$ \\
\hline $\begin{array}{l}\text { Estimated Kink } \\
\text { (conventional std error) }\end{array}$ & $\begin{array}{c}2.2 \\
(0.1)\end{array}$ & $\begin{array}{c}2.2 \\
(0.3)\end{array}$ & $\begin{array}{l}-1.9 \\
(0.4)\end{array}$ & $\begin{array}{l}-1.4 \\
(0.2)\end{array}$ \\
\hline $\begin{array}{l}\text { Estimated Kink - Bias Corrected } \\
\text { [robust confidence interval] }\end{array}$ & $\begin{array}{l}2.4 \\
{[1.7,3.1]}\end{array}$ & $\begin{array}{c}1.9 \\
{[0.4,3.4]}\end{array}$ & $\begin{array}{c}-1.4 \\
{[-2.1,-0.7]}\end{array}$ & $\begin{array}{c}-0.8 \\
{[-1.9,0.3]}\end{array}$ \\
\hline \multicolumn{5}{|c|}{ B. Reduced Form Model (Dependent variable $=\log$ time to next jobt) } \\
\hline \multicolumn{5}{|l|}{ Default CCT (with regularization) } \\
\hline $\begin{array}{l}\text { Estimated Kink } \\
\text { (conventional std error) }\end{array}$ & $\begin{array}{c}2.2 \\
(6.7)\end{array}$ & $\begin{array}{c}27.0 \\
(23.0)\end{array}$ & $\begin{array}{c}2.5 \\
(3.4)\end{array}$ & $\begin{array}{c}9.2 \\
(6.7)\end{array}$ \\
\hline $\begin{array}{l}\text { Estimated Kink - Bias Corrected } \\
\text { [robust confidence interval] }\end{array}$ & $\begin{array}{c}1.3 \\
{[-19.5,22.0]}\end{array}$ & $\begin{array}{c}38.5 \\
{[-20,97]}\end{array}$ & $\begin{array}{c}3.7 \\
{[-6.5,13.9]}\end{array}$ & $\begin{array}{c}11.0 \\
{[-6.9,28.9]}\end{array}$ \\
\hline \multicolumn{5}{|l|}{ CCT with no regularization } \\
\hline Main (Pilot) Bandwidth & $1,330(1,574)$ & $4,736(1,971)$ & $2,825(3,053)$ & $4,225(4,997)$ \\
\hline $\begin{array}{l}\text { Estimated Kink } \\
\text { (conventional std error) }\end{array}$ & $\begin{array}{c}3.1 \\
(1.7)\end{array}$ & $\begin{array}{c}5.5 \\
(2.3)\end{array}$ & $\begin{array}{l}-3.0 \\
(1.4)\end{array}$ & $\begin{array}{l}-1.4 \\
(3.1)\end{array}$ \\
\hline $\begin{array}{l}\text { Estimated Kink - Bias Corrected } \\
\text { [robust confidence interval] }\end{array}$ & $\begin{array}{c}0.9 \\
{[-9.7,11.5]}\end{array}$ & $\begin{array}{c}-24.5 \\
{[-90,41]}\end{array}$ & $\begin{array}{c}-1.3 \\
{[-9.5,7.0]}\end{array}$ & $\begin{array}{c}1.4 \\
{[-10.8,13.5]}\end{array}$ \\
\hline \multicolumn{5}{|l|}{$F G$} \\
\hline Main (Pilot) Bandwidth & $2,501(3,858)$ & $4,259(4,918)$ & $4,465(7,255)$ & $8,001(12,383)$ \\
\hline $\begin{array}{l}\text { Estimated Kink } \\
\text { (conventional std error) }\end{array}$ & $\begin{array}{c}3.0 \\
(0.8)\end{array}$ & $\begin{array}{c}4.8 \\
(2.4)\end{array}$ & $\begin{array}{l}-3.0 \\
(0.7)\end{array}$ & $\begin{array}{l}-4.1 \\
(1.5)\end{array}$ \\
\hline $\begin{array}{l}\text { Estimated Kink - Bias Corrected } \\
\text { [robust confidence interval] }\end{array}$ & $\begin{array}{c}4.2 \\
{[-1.4,9.8]}\end{array}$ & $\begin{array}{c}9.9 \\
{[-3.6,23.4]}\end{array}$ & $\begin{array}{c}-5.2 \\
{[-8.4,-1.9]}\end{array}$ & $\begin{array}{c}0.0 \\
{[-7.0,6.9]}\end{array}$ \\
\hline
\end{tabular}


Table 2b: Bias and Variance Tradeoff -- Conventional versus Bias-corrected Estimators

\begin{tabular}{cccccc}
\multicolumn{2}{c}{ Bottom Kink } & & \multicolumn{2}{c}{ Top Kink } \\
\cline { 1 - 1 } \cline { 5 - 6 } Local Linear & Local Quad. & & Local Linear & Local Quad. \\
$(1)$ & $(2)$ & & (3) & (3)
\end{tabular}

A. First Stage Model (Dependent variable $=\log$ daily benefit)

Default CCT (with regularization)

$\begin{array}{lllll}\text { Estimated Bias } & 0.6 & 0.9 & 0.2 & 1.3 \\ \text { Estimated Bias Squared } & 0.3 & 0.8 & 0.0 & 1.6 \\ \text { Change in Estimated Variance } & 1.2 & 1.7 & 0.6 & 3.0\end{array}$

CCT with no regularization

Estimated Bias

Estimated Bias Squared

Change in Estimated Variance

$\begin{array}{llll}0.3 & 0.8 & -0.2 & 1.2 \\ 0.1 & 0.6 & 0.0 & 1.4 \\ 0.2 & 1.2 & 0.3 & 2.2\end{array}$

FG

Estimated Bias

Estimated Bias Squared

Change in Estimated Variance

$-0.1$

0.3

$-0.2$

0.1

$-0.5$

0.1

0.5

B. Reduced Form Model (Dependent variable $=\log$ time to next jobt) Default CCT (with regularization)

$\begin{array}{lcccc}\text { Estimated Bias } & 1.0 & -11.5 & -1.3 & -2.1 \\ \text { Estimated Bias Squared } & 0.9 & 132.3 & 1.7 & 4.4 \\ \text { Change in Estimated Variance } & 67.2 & 361.8 & 15.5 & 38.5\end{array}$

CCT with no regularization

$\begin{array}{lcccc}\text { Estimated Bias } & 2.2 & 29.4 & -1.8 & -2.8 \\ \text { Estimated Bias Squared } & 4.8 & 864.4 & 3.1 & 7.6 \\ \text { Change in Estimated Variance } & 26.4 & 1108.9 & 15.8 & 28.8\end{array}$

Change in Estimated Variance

26.4

1108.9

15.8

28.8

FG

$\begin{array}{lllll}\text { Estimated Bias } & -1.3 & -5.5 & 2.2 & -4.4\end{array}$

$\begin{array}{lllll}\text { Estimated Bias Squared } & 1.7 & 30.3 & 4.6 & 18.9\end{array}$

$\begin{array}{lllll}\text { Change in Estimated Variance } & 7.5 & 39.6 & 2.3 & 10.0\end{array}$

Notes: Estimated bias is the difference between the value of the conventional linear estimator and the its biascorrected counterpart, which is the center of the robust confidence interval. The change in estimated variance is

the difference between the variance of the bias corrected estimator and its conventional counterpart. Estimated bias is multiplied by $10^{5}$, and estimated bias squared and change in estimated variance are both multiplied by $10^{10}$. 
Table 3: Estimates of Kinks in Conditional Means of Covariates

\begin{tabular}{|c|c|c|c|c|c|c|}
\hline & \multicolumn{3}{|c|}{ Local Linear Models } & \multicolumn{3}{|c|}{ Local Quadratic Models } \\
\hline & $\begin{array}{c}\text { FG } \\
\text { Bandwidth } \\
\text { (1) }\end{array}$ & $\begin{array}{c}\text { Estimated } \\
\text { Kink } \\
(2)\end{array}$ & $\begin{array}{c}\text { Bias } \\
\text { Corrected } \\
\text { Kink } \\
(3) \\
\end{array}$ & $\begin{array}{c}\text { FG } \\
\text { Bandwidth } \\
(4)\end{array}$ & $\begin{array}{c}\text { Estimated } \\
\text { Kink } \\
(5)\end{array}$ & $\begin{array}{c}\text { Bias } \\
\text { Corrected } \\
\text { Kink } \\
(6)\end{array}$ \\
\hline \multicolumn{7}{|l|}{ A. Bottom Kink: } \\
\hline $\begin{array}{l}\text { Predicted log time } \\
\text { to next job }\end{array}$ & 3,344 & $\begin{array}{c}0.9 \\
(0.2)\end{array}$ & $\begin{array}{c}0.8 \\
{[-1.0,2.5]}\end{array}$ & 4,564 & $\begin{array}{c}1.2 \\
(0.8)\end{array}$ & $\begin{array}{c}4.0 \\
{[-0.4,8.3]}\end{array}$ \\
\hline Female & 1,094 & $\begin{array}{c}0.6 \\
(1.0)\end{array}$ & $\begin{array}{c}-1.1 \\
{[-4.0,1.9]}\end{array}$ & 3,440 & $\begin{array}{l}-0.3 \\
(1.2)\end{array}$ & $\begin{array}{c}-2.1 \\
{[-8.1,4.0]}\end{array}$ \\
\hline Age & 1,627 & $\begin{array}{l}29.5 \\
(9.8)\end{array}$ & $\begin{array}{c}46.8 \\
{[-0.4,94]}\end{array}$ & 3,311 & $\begin{array}{c}32.9 \\
(20.9)\end{array}$ & $\begin{array}{c}-16.5 \\
{[-122,89]}\end{array}$ \\
\hline Blue collar occup. & 2,530 & $\begin{array}{l}-1.5 \\
(0.3)\end{array}$ & $\begin{array}{c}-1.8 \\
{[-4.1,0.6]}\end{array}$ & 4,564 & $\begin{array}{l}-1.5 \\
(1.0)\end{array}$ & $\begin{array}{c}-6.3 \\
{[-12.1,-0.4]}\end{array}$ \\
\hline Recalled to last job & 3,199 & $\begin{array}{l}-0.8 \\
(0.3)\end{array}$ & $\begin{array}{c}0.5 \\
{[-1.9,2.9]}\end{array}$ & 3,194 & $\begin{array}{c}0.5 \\
(1.1)\end{array}$ & $\begin{array}{c}-2.9 \\
{[-8.1,2.4]}\end{array}$ \\
\hline \multicolumn{7}{|l|}{ B. Top Kink: } \\
\hline $\begin{array}{l}\text { Predicted log time } \\
\text { to next job }\end{array}$ & 13,708 & $\begin{array}{c}0.0 \\
(0.1)\end{array}$ & $\begin{array}{c}-1.5 \\
{[-3.0,0.01]}\end{array}$ & 8,095 & $\begin{array}{l}-1.2 \\
(0.5)\end{array}$ & $\begin{array}{c}-1.9 \\
{[-4.3,0.6]}\end{array}$ \\
\hline Female & 5,364 & $\begin{array}{l}-0.2 \\
(0.2)\end{array}$ & $\begin{array}{c}-0.9 \\
{[-1.9,0.2]}\end{array}$ & 13,908 & $\begin{array}{l}-1.2 \\
(0.4)\end{array}$ & $\begin{array}{c}-1.5 \\
{[-4,1.1]}\end{array}$ \\
\hline Age & 6,160 & $\begin{array}{l}-4.6 \\
(2.6)\end{array}$ & $\begin{array}{c}-5.3 \\
{[-23.1,12.6]}\end{array}$ & 9,776 & $\begin{array}{l}-6.1 \\
(7.8)\end{array}$ & $\begin{array}{c}-14.0 \\
{[-55.0,27.0]}\end{array}$ \\
\hline Blue collar occup. & 2,067 & $\begin{array}{c}1.5 \\
(1.0)\end{array}$ & $\begin{array}{c}1.8 \\
{[-0.7,4.3]}\end{array}$ & 5,401 & $\begin{array}{c}2.6 \\
(0.9)\end{array}$ & $\begin{array}{c}4.0 \\
{[0.7,7.2]}\end{array}$ \\
\hline Recalled to last job & 5,013 & $\begin{array}{c}0.6 \\
(0.2)\end{array}$ & $\begin{array}{c}1.1 \\
{[-0.02,2.2]}\end{array}$ & 13,908 & $\begin{array}{c}2.7 \\
(0.4)\end{array}$ & $\begin{array}{c}1.1 \\
{[-1.6,3.7]}\end{array}$ \\
\hline
\end{tabular}

Notes: standard errors in parentheses, robust confidence intervals in square brackets. See notes to Table 2. All estimates are multiplied by $10^{5}$. Predicted log time to next job is the estimated covariate index for this outcome, fit on the pooled bottom and top kink samples. The vector of 59 covariates includes dummies for gender, blue collar occupation, and being recalled to the previous job, decile of age ( 9 dummies), decile of previous job tenure ( 9 dummies), quintile of previous daily wage (4 dummies), major industry ( 6 dummies), region (3 dummies), year of claim (7 dummies), decile of previous firm size (9 dummies), and decile of previous firm's recall rate ( 9 dummies). 
Table 4: Estimated Elasticities of Joblessness Duration from Fuzzy Regression Kink Design, FG Bandwidth

\begin{tabular}{|c|c|c|c|c|c|c|}
\hline & \multicolumn{3}{|c|}{ Local Linear Models } & \multicolumn{3}{|c|}{ Local Quadratic Models } \\
\hline & $\begin{array}{c}\text { FG Bandwidth } \\
\text { Main (Pilot) } \\
(1)\end{array}$ & $\begin{array}{c}\text { Estimated } \\
\text { Elasticity (std. } \\
\text { error) } \\
\text { (2) }\end{array}$ & $\begin{array}{c}\text { Bias-Corrected } \\
\text { Estimate } \\
\text { [Robust } \mathrm{Cl}] \\
\text { (3) }\end{array}$ & $\begin{array}{c}\text { FG Bandwidth } \\
\text { Main (Pilot) } \\
(4)\end{array}$ & $\begin{array}{c}\text { Estimated } \\
\text { Elasticity (std. } \\
\text { error) } \\
(5) \\
\end{array}$ & $\begin{array}{c}\text { Bias-Corrected } \\
\text { Estimate } \\
\text { [Robust CI] } \\
(6)\end{array}$ \\
\hline \multicolumn{7}{|l|}{ A. Bottom Kink: } \\
\hline & $2,501(3,858)$ & $\begin{array}{c}1.37 \\
(0.37)\end{array}$ & $\begin{array}{c}1.95 \\
{[-0.59,4.49]}\end{array}$ & $4,259(4,918)$ & $\begin{array}{c}2.35 \\
(1.20)\end{array}$ & $\begin{array}{c}5.04 \\
{[-1.87,11.94]}\end{array}$ \\
\hline \multicolumn{7}{|l|}{ B. Top Kink: } \\
\hline & $4,465(7,255)$ & $\begin{array}{c}2.04 \\
(0.52)\end{array}$ & $\begin{array}{c}3.67 \\
{[1.24,6.09]}\end{array}$ & $8,011(12,383)$ & $\begin{array}{c}2.71 \\
(1.07)\end{array}$ & $\begin{array}{c}1.20 \\
{[-4.51,6.90]}\end{array}$ \\
\hline
\end{tabular}

Notes: Standard errors in parentheses. FG bandwidth is derived based on the outcome variable. Point estimates and standard errors are obtained from 2SLS regressions described in Card et al (2012). Robust Cl's are obtained by a variant of the Stata package described in Calonico et al (in press). 
Table 5: Estimates of Benefit Elasticity (Fuzzy RK), Alternative Estimators and Bandwidths

\begin{tabular}{|c|c|c|c|c|c|c|c|c|}
\hline & \multicolumn{4}{|c|}{ Bottom Kink } & \multicolumn{4}{|c|}{ Top Kink } \\
\hline & \multicolumn{2}{|c|}{ Local Linear } & \multicolumn{2}{|c|}{ Local Quadratic } & \multicolumn{2}{|c|}{ Local Linear } & \multicolumn{2}{|c|}{ Local Quadratic } \\
\hline & $\begin{array}{c}\text { First Stage } \\
\left(\text { Coeff } \times 10^{5}\right) \\
(1)\end{array}$ & $\begin{array}{c}\text { Struct. Model } \\
\text { (2) }\end{array}$ & $\begin{array}{c}\text { First Stage } \\
\left(\text { Coeff } \times 10^{5}\right) \\
(3)\end{array}$ & $\begin{array}{l}\text { Struct. Model } \\
\text { (4) } \\
\end{array}$ & $\begin{array}{c}\text { First Stage } \\
\left(\text { Coeff } \times 10^{5}\right) \\
(5)\end{array}$ & $\begin{array}{c}\text { Struct. Model } \\
(6) \\
\end{array}$ & $\begin{array}{c}\text { First Stage } \\
\left(\text { Coeff } \times 10^{5}\right) \\
(7)\end{array}$ & $\begin{array}{c}\text { Struct. Model } \\
\text { (8) } \\
\end{array}$ \\
\hline \multicolumn{9}{|c|}{ Default CCT (with regularization) } \\
\hline Main Bandwidth (Pilot) & \multicolumn{2}{|c|}{$537(1062)$} & \multicolumn{2}{|c|}{$598(985)$} & \multicolumn{2}{|c|}{$1593(3188)$} & \multicolumn{2}{|c|}{2546 (4015) } \\
\hline $\begin{array}{l}\text { Estimated Kink } \\
\text { (conventional std error) }\end{array}$ & $\begin{array}{l}2.0 \\
(0.7)\end{array}$ & $\begin{array}{c}1.1 \\
(3.4)\end{array}$ & $\begin{array}{c}1.2 \\
(2.4)\end{array}$ & $\begin{array}{c}22.5 \\
(51.4)\end{array}$ & $\begin{array}{l}-1.9 \\
(0.5)\end{array}$ & $\begin{array}{l}-1.3 \\
(1.8)\end{array}$ & $\begin{array}{l}-1.6 \\
(1.0)\end{array}$ & $\begin{array}{l}-5.7 \\
(5.5)\end{array}$ \\
\hline Bias-corrected Estimate & 1.6 & 5.2 & 1.9 & 19.0 & -1.6 & -2.2 & -1.6 & -6.9 \\
\hline [robust conf. interval] & {$[-0.6,3.7]$} & {$[-9.6,11.4]$} & {$[-4.3,8.1]$} & {$[-110,148]$} & {$[-3.2,0.1]$} & {$[-7.7,3.3]$} & {$[-4.4,1.2]$} & {$[-21.6,7.9]$} \\
\hline \multicolumn{9}{|l|}{ CCT with no regularization } \\
\hline Main Bandwidth (Pilot) & \multicolumn{2}{|c|}{$1330(1574)$} & \multicolumn{2}{|c|}{4736 (1971) } & \multicolumn{2}{|c|}{$2825(3503)$} & \multicolumn{2}{|c|}{$4225(4997)$} \\
\hline $\begin{array}{l}\text { Estimated Kink } \\
\text { (conventional std error) }\end{array}$ & $\begin{array}{c}2.1 \\
(0.2)\end{array}$ & $\begin{array}{c}1.5 \\
(0.8)\end{array}$ & $\begin{array}{c}2.0 \\
(0.3)\end{array}$ & $\begin{array}{c}2.7 \\
(1.2)\end{array}$ & $\begin{array}{l}-1.1 \\
(0.2)\end{array}$ & $\begin{array}{c}2.7 \\
(1.4)\end{array}$ & $\begin{array}{l}-0.8 \\
(0.5)\end{array}$ & $\begin{array}{c}1.6 \\
(3.8)\end{array}$ \\
\hline $\begin{array}{l}\text { Bias-corrected Estimate } \\
\text { [robust conf. interval] }\end{array}$ & $\begin{array}{c}1.9 \\
{[0.7,3.0]}\end{array}$ & $\begin{array}{c}0.6 \\
{[-4.5,5.7]}\end{array}$ & $\begin{array}{c}-0.3 \\
{[-6.9,6.4]}\end{array}$ & $\begin{array}{c}-9.5 \\
{[-43,24]}\end{array}$ & $\begin{array}{c}-0.9 \\
{[-2.1,0.4]}\end{array}$ & $\begin{array}{c}1.8 \\
{[-6.6,10.2]}\end{array}$ & $\begin{array}{c}-1.1 \\
{[-2.9,0.8]}\end{array}$ & $\begin{array}{c}-2.3 \\
{[-18.2,13.6]}\end{array}$ \\
\hline \multicolumn{9}{|l|}{ Fuzzy CCT (no regularization) } \\
\hline Main Bandwidth (Pilot) & \multicolumn{2}{|c|}{$1074(1412)$} & \multicolumn{2}{|c|}{$1558(1595)$} & \multicolumn{2}{|c|}{$4567(3781)$} & \multicolumn{2}{|c|}{$4208(7740)$} \\
\hline $\begin{array}{l}\text { Estimated Kink } \\
\text { (conventional std error) }\end{array}$ & $\begin{array}{c}2.3 \\
(0.3)\end{array}$ & $\begin{array}{c}1.1 \\
(1.0)\end{array}$ & $\begin{array}{l}1.8 \\
(0.6)\end{array}$ & $\begin{array}{c}0.1 \\
(3.0)\end{array}$ & $\begin{array}{l}-1.4 \\
(0.1)\end{array}$ & $\begin{array}{l}2.0 \\
(0.5)\end{array}$ & $\begin{array}{l}-0.8 \\
(0.5)\end{array}$ & $\begin{array}{c}1.9 \\
(3.8)\end{array}$ \\
\hline $\begin{array}{l}\text { Bias-corrected Estimate } \\
\text { [robust conf. interval] }\end{array}$ & $\begin{array}{c}2.1 \\
{[0.7,3.4]}\end{array}$ & $\begin{array}{c}2.4 \\
{[-3.1,7.8]}\end{array}$ & $\begin{array}{c}1.7 \\
{[-1.1,4.4]}\end{array}$ & $\begin{array}{c}4.8 \\
{[-9.5,19]}\end{array}$ & $\begin{array}{c}-0.8 \\
{[-2.1,0.5]}\end{array}$ & $\begin{array}{c}3.0 \\
{[-3.4,9.3]}\end{array}$ & $\begin{array}{c}-0.5 \\
{[-1.8,0.9]}\end{array}$ & $\begin{array}{c}1.3 \\
{[-9.8,12.3]}\end{array}$ \\
\hline \multicolumn{9}{|l|}{ Fuzzy IK (no regularization) } \\
\hline Main Bandwidth (Pilot) & \multicolumn{2}{|c|}{1058 (1679) } & \multicolumn{2}{|c|}{$1887(1725)$} & \multicolumn{2}{|c|}{3125 (4909) } & \multicolumn{2}{|c|}{5796 (5115) } \\
\hline $\begin{array}{l}\text { Estimated Kink } \\
\text { (conventional std error) }\end{array}$ & $\begin{array}{c}2.3 \\
(0.3)\end{array}$ & $\begin{array}{c}1.3 \\
(1.1)\end{array}$ & $\begin{array}{l}2.0 \\
(0.4)\end{array}$ & $\begin{array}{c}0.6 \\
(2.0)\end{array}$ & $\begin{array}{l}-1.3 \\
(0.2)\end{array}$ & $\begin{array}{c}2.7 \\
(1.1)\end{array}$ & $\begin{array}{l}-1.3 \\
(0.3)\end{array}$ & $\begin{array}{c}3.5 \\
(1.7)\end{array}$ \\
\hline $\begin{array}{l}\text { Bias-corrected Estimate } \\
\text { [robust conf. interval] }\end{array}$ & $\begin{array}{c}2.2 \\
{[1.1,3.2]}\end{array}$ & $\begin{array}{c}1.2 \\
{[-3.1,5.4]}\end{array}$ & $\begin{array}{c}2.0 \\
{[-1.0,5.0]}\end{array}$ & $\begin{array}{c}3.4 \\
{[-9.6,16.4]}\end{array}$ & $\begin{array}{c}-0.8 \\
{[-1.5,0.0]}\end{array}$ & $\begin{array}{c}3.2 \\
{[-1.2,7.6]}\end{array}$ & $\begin{array}{c}-1.1 \\
{[-3.2,1.1]}\end{array}$ & $\begin{array}{c}0.1 \\
{[-12.5,12.7]}\end{array}$ \\
\hline
\end{tabular}

Notes: Point estimates and standard errors are obtained from 2SLS regressions described in Card et al (2012). The default CCT bandwidth, CCT with no regularization and the Robust Cl's are obtained by a variant of the Stata package described in Calonico et al (in press). The fuzzy CCT and fuzzy IK bandwidths are authors' calculations. 
Table 6a: Summary of Monte Carlo Studies, DGP Design Based on Bottom Kink Sample

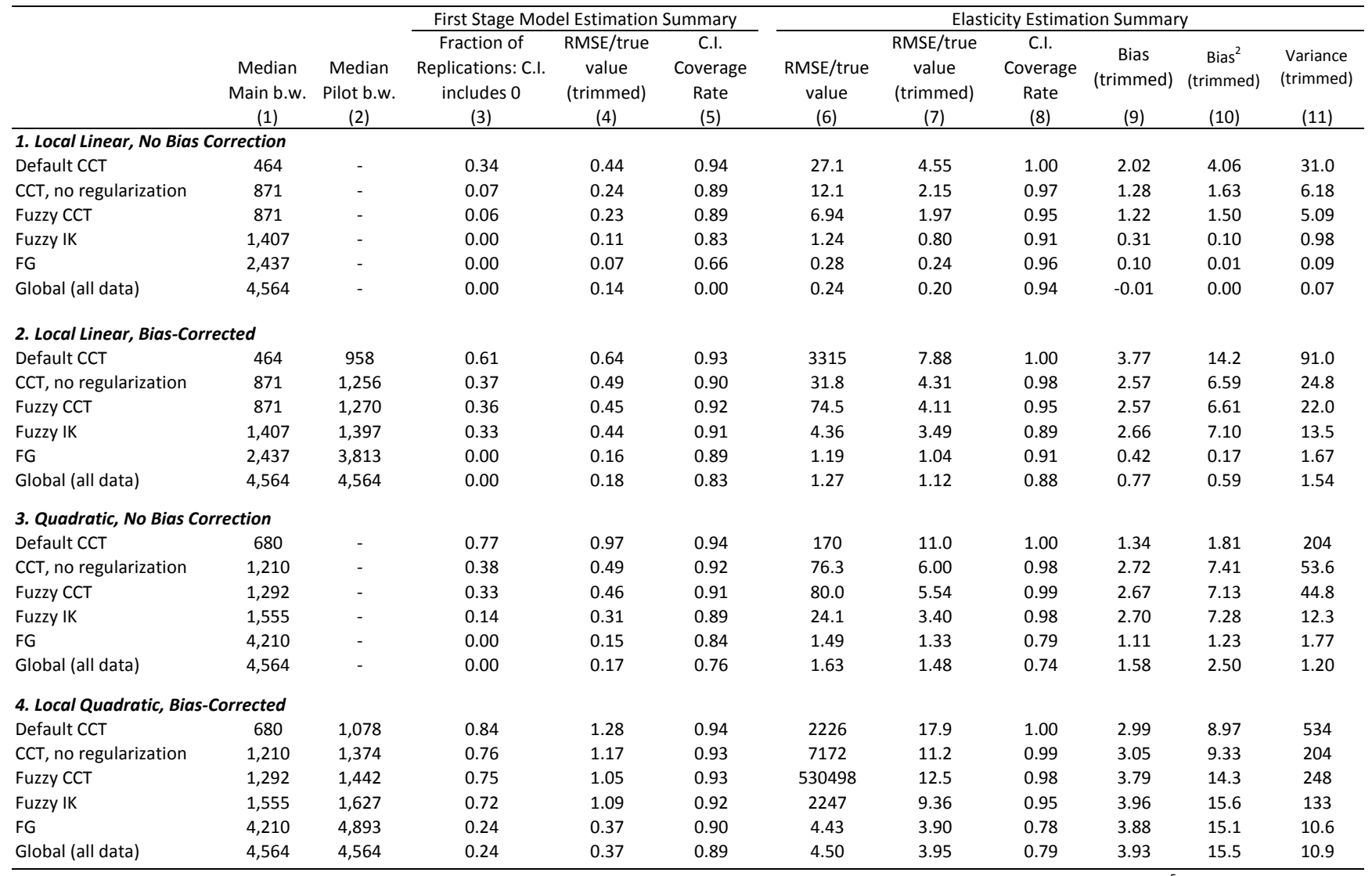

Notes: based on 1,000 simulations. DGP is based on 5 th order polynomial approximation of bottom kink sample. True kink in first stage is: $2.3 \times 10^{-5}$. True elasticity is: 1.3 . The trimmed statistic are obtained by first trimming the $5 \%$ sample in which the estimates deviate the most from the true parameter value. 
Table 6b: Summary of Monte Carlo Studies, DGP Design Based on Top Kink Sample

\begin{tabular}{|c|c|c|c|c|c|c|c|c|c|c|c|}
\hline & \multirow[b]{2}{*}{$\begin{array}{l}\text { Median } \\
\text { Main b.w. } \\
(1)\end{array}$} & \multirow[b]{2}{*}{$\begin{array}{l}\text { Median } \\
\text { Pilot b.w. } \\
\text { (2) }\end{array}$} & \multicolumn{3}{|c|}{ First Stage Model Estimation Summary } & \multicolumn{6}{|c|}{ Elasticity Estimation Summary } \\
\hline & & & $\begin{array}{c}\text { Fraction of } \\
\text { Replications: C.I. } \\
\text { includes } 0 \\
\text { (3) } \\
\end{array}$ & $\begin{array}{l}\text { RMSE/true } \\
\text { value } \\
\text { (trimmed) } \\
\text { (4) }\end{array}$ & $\begin{array}{c}\text { C.I. } \\
\text { Coverage } \\
\text { Rate } \\
\text { (5) } \\
\end{array}$ & $\begin{array}{l}\text { RMSE/true } \\
\text { value } \\
(6)\end{array}$ & $\begin{array}{l}\text { RMSE/true } \\
\text { value } \\
\text { (trimmed) } \\
(7) \\
\end{array}$ & $\begin{array}{c}\text { C.I. } \\
\text { Coverage } \\
\text { Rate } \\
(8) \\
\end{array}$ & $\begin{array}{c}\text { Bias } \\
\text { (trimmed) } \\
\text { (9) }\end{array}$ & $\begin{array}{c}\text { Bias }^{2} \\
\text { (trimmed) } \\
(10)\end{array}$ & $\begin{array}{c}\text { Variance } \\
\text { (trimmed) } \\
(11)\end{array}$ \\
\hline \multicolumn{12}{|c|}{ 1. Local Linear, No Bias Correction } \\
\hline Default CCT & 1,371 & - & 0.62 & 0.59 & 0.81 & 78.8 & 3.66 & 0.99 & 0.50 & 0.25 & 53.5 \\
\hline $\mathrm{CCT}$, no regularization & 2,683 & - & 0.15 & 0.34 & 0.72 & 7.37 & 1.25 & 0.91 & -0.02 & 0.00 & 6.30 \\
\hline Fuzzy CCT & 2,301 & - & 0.25 & 0.43 & 0.72 & 81.0 & 1.97 & 0.94 & 0.04 & 0.00 & 15.5 \\
\hline Fuzzy IK & 3,254 & - & 0.00 & 0.20 & 0.74 & 0.76 & 0.52 & 0.94 & 0.39 & 0.15 & 0.94 \\
\hline FG & 4,465 & - & 0.00 & 0.15 & 0.77 & 0.48 & 0.36 & 0.82 & 0.11 & 0.01 & 0.52 \\
\hline Global (all data) & 13,908 & - & 0.00 & 0.55 & 0.00 & 0.81 & 0.80 & 0.00 & -1.60 & 2.56 & 0.01 \\
\hline \multicolumn{12}{|c|}{ 2. Local Linear, Bias-Corrected } \\
\hline Default CCT & 1,371 & 2,842 & 0.88 & 0.90 & 0.79 & 12323 & 10.1 & 0.99 & 2.32 & 5.40 & 400 \\
\hline $\mathrm{CCT}$, no regularization & 2,683 & 3,673 & 0.68 & 0.76 & 0.69 & 473 & 3.12 & 0.93 & 0.01 & 0.00 & 39.1 \\
\hline Fuzzy CCT & 2,301 & 3,570 & 0.68 & 0.79 & 0.69 & 18102 & 5.12 & 0.95 & 0.61 & 0.38 & 104 \\
\hline Fuzzy IK & 3,254 & 4,604 & 0.44 & 0.59 & 0.64 & 2.36 & 1.67 & 0.90 & 0.77 & 0.59 & 10.5 \\
\hline FG & 4,465 & 7,300 & 0.00 & 0.19 & 0.88 & 1.17 & 0.95 & 0.81 & 1.50 & 2.26 & 1.36 \\
\hline Global (all data) & 13,908 & 13,908 & 0.00 & 0.22 & 0.72 & 0.92 & 0.87 & 0.44 & 1.57 & 2.45 & 0.55 \\
\hline \multicolumn{12}{|c|}{ 3. Quadratic, No Bias Correction } \\
\hline Default CCT & 2,033 & - & 0.90 & 1.10 & 0.89 & 93.6 & 7.95 & 1.00 & -2.04 & 4.18 & 249 \\
\hline $\mathrm{CCT}$, no regularization & 3,459 & - & 0.66 & 0.77 & 0.72 & 52.6 & 6.70 & 0.96 & -0.39 & 0.15 & 180 \\
\hline Fuzzy CCT & 3,613 & - & 0.64 & 0.72 & 0.69 & 97.2 & 6.00 & 0.97 & -0.59 & 0.35 & 144 \\
\hline Fuzzy IK & 4,964 & - & 0.34 & 0.49 & 0.71 & 43.4 & 3.03 & 0.92 & 0.26 & 0.07 & 36.7 \\
\hline FG & 8,060 & - & 0.00 & 0.15 & 0.91 & 1.06 & 0.93 & 0.84 & 1.59 & 2.53 & 0.94 \\
\hline Global (all data) & 13,908 & - & 0.00 & 0.20 & 0.58 & 2.07 & 1.97 & 0.03 & 3.79 & 14.3 & 1.16 \\
\hline \multicolumn{12}{|c|}{ 4. Local Quadratic, Bias-Corrected } \\
\hline Default CCT & 2,033 & 3,232 & 0.91 & 1.40 & 0.90 & 1013 & 15.0 & 1.00 & -1.40 & 1.96 & 904 \\
\hline $\mathrm{CCT}$, no regularization & 3,459 & 4,088 & 0.91 & 1.42 & 0.78 & 21785 & 26.5 & 0.99 & 1.25 & 1.55 & 2803 \\
\hline Fuzzy CCT & 3,613 & 4,264 & 0.92 & 2.17 & 0.79 & 16114 & 25.9 & 0.98 & 3.77 & 14.2 & 2677 \\
\hline Fuzzy IK & 4,964 & 5,033 & 0.93 & 1.44 & 0.68 & 65325 & 14.3 & 0.93 & 1.03 & 1.05 & 821 \\
\hline FG & 8,060 & 12,471 & 0.71 & 0.63 & 0.62 & 1.89 & 1.53 & 0.93 & 0.36 & 0.13 & 9.2 \\
\hline Global (all data) & 13,908 & 13,908 & 0.79 & 0.71 & 0.49 & 2.36 & 1.89 & 0.93 & 0.94 & 0.88 & 13.5 \\
\hline
\end{tabular}

Notes: based on 1,000 simulations. DGP is based on 5th order polynomial approximation of top kink sample. True kink in first stage is: $-1.4 \times 10^{-5}$. True elasticity is: 2.0 . The trimmed statistic are obtained by first trimming the $5 \%$ sample in which the estimates deviate the most from the true parameter value. 


\section{Supplemental Appendix}

\section{A Identification}

\section{A.1 Proofs of Proposition 1 and 2}

In order to prove Proposition 1, we first present and prove the following Lemmas.

Lemma 1. Let $\varphi(x, t): I \times[c, d] \rightarrow R$ where $I$ is a compact subset of $\mathbb{R}^{m}$. Suppose $\varphi(x, t)$ and its partial derivative, $\varphi_{2}(x, t)$, are continuous and that $\varphi$ is integrable with respect to the probability measure $\alpha$ for each $t$. Then $f(t)=\int \varphi(x, t) d \alpha(x)$ is continuously differentiable on $[c, d]$.

Proof: By Theorem 5 (p.97) of Roussas (2004), $f^{\prime}(t)=\int \varphi_{2}(x, t) d \alpha(x)$ for all $t \in[c, d]$. Let $s_{1}, s_{2} \in[c, d]$

$$
\begin{aligned}
\left|f^{\prime}\left(s_{1}\right)-f^{\prime}\left(s_{2}\right)\right| & =\left|\int \varphi_{2}\left(x, s_{1}\right) d \alpha(x)-\int \varphi_{2}\left(x, s_{2}\right) d \alpha(x)\right| \\
& \leqslant \int\left|\varphi_{2}\left(x, s_{1}\right)-\varphi_{2}\left(x, s_{2}\right)\right| d \alpha(x) .
\end{aligned}
$$

The continuity of $\varphi_{2}(x, t)$ on the compact set $I \times[c, d]$ implies uniform continuity, and therefore we can choose a $\delta$ such that $\left|s_{1}-s_{2}\right|<\delta \mid$ implies $\left|\varphi_{2}\left(x, s_{1}\right)-\varphi_{2}\left(x, s_{2}\right)\right|<\frac{\varepsilon}{\alpha(I)}$ for all $x \in I$, which in turn implies that $\left|f^{\prime}\left(s_{1}\right)-f^{\prime}\left(s_{2}\right)\right|<\varepsilon$. QED.

Lemma 2. Under Assumptions 1(i), 3(ii) and 4, $f(v)$ is continuously differentiable and strictly positive on $I_{V}$.

Proof: The continuous differentiability of $f(v)$ follows from Assumption 1(i), Assumption 4 and Lemma 1. $f(V) \geqslant \int_{A_{U}} f_{V \mid U=u}(v) d F_{U}(u)>0$ follows directly from Assumption 3(ii).

In order to prove Proposition 2, we present and prove the following lemmas. Let $S$ be a sub-vector of the random vector $\left(U, \varepsilon, U_{B^{\prime}}, U_{V^{\prime}}\right)$ that at least includes $U$ and $\varepsilon$, and let $S^{*}$ denote the vector of the random variables in $\left(U, \varepsilon, U_{B^{\prime}}, U_{V^{\prime}}\right)$ but not in $S$. Let $Q$ be a sub-vector of the random vector $\left(\varepsilon, U_{B^{\prime}}, U_{V^{\prime}}\right)$ that at least includes $\varepsilon$, and let $Q^{*}$ denote the vector of the random variables in $\left(U, \varepsilon, U_{B^{\prime}}, U_{V^{\prime}}\right)$ but not in $Q$. Let $I_{V^{*}}, I_{S}$, $I_{S^{*}}, I_{Q}$ and $I_{Q^{*}}$ be the smallest closed rectangle that contains the support of $V^{*}, S, S^{*}, Q$ and $Q^{*}$, respectively. In the proofs, we only consider the case where $\pi^{i j}\left(V, U, \varepsilon, U_{V^{\prime}}, U_{B^{\prime}}\right)>0$ for all $i, j=0,1$ since the proofs for the cases where some of the $\pi^{i j}=0$ are similar in spirit and simpler. Also, we abstract away in the following 
lemmas from the potential issues in situations where the conditioning set, e.g. $S=s$, is of measure 0 (cf Borel-Kolmogorov paradox) because the integrals in Proposition is 2 are over the distribution of $S$.

Lemma 3. a) $f_{S^{*} \mid S=s}\left(s^{*}\right)$ is continuous on $I_{S^{*}, S}$; b) $f_{Q^{*} \mid Q=q}\left(q^{*}\right)$ is continuous on $I_{Q^{*}, Q}$.

Proof: We prove part a), and the proof for part b) is similar. There are three cases: 1) $S^{*}=U_{V^{\prime}}, 2$ ) $S^{*}=U_{B^{\prime}}$ and 3) $S^{*}=\left(U_{V^{\prime}}, U_{B^{\prime}}\right)$. For case 1$)$,

$$
\begin{aligned}
f_{U_{V^{\prime}} \mid U_{B^{\prime}}=u_{B^{\prime}}, U=u, \varepsilon=e}\left(u_{V^{\prime}}\right) & =\frac{f_{U_{V^{\prime}}, U_{B^{\prime}} \mid U=u, \varepsilon=e}\left(u_{V^{\prime}}, u_{B^{\prime}}\right)}{f_{U_{B^{\prime}} \mid U=u, \varepsilon=e}\left(u_{B^{\prime}}\right)} \\
& =\frac{\int f_{V, U_{V^{\prime}}, U_{B^{\prime}}} \mid U=u, \varepsilon=e}{\iint f_{V, U_{V^{\prime}}, U_{B^{\prime}} \mid U=u, \varepsilon=e}\left(v, u_{V^{\prime}}, u_{B^{\prime}}\right) d v} .
\end{aligned}
$$

Note that the numerator of (10) is exactly $f_{S^{*} \mid S=s}\left(s^{*}\right)$ in case 3$)$. Both the numerator and the denominator are continuous as guaranteed by Assumption 4a and Proposition 1 in 17.5 of Zorich (2004). Since the denominator is strictly positive, $f_{U_{V^{\prime}} \mid U_{B^{\prime}}=u_{B^{\prime}}, U=u, \varepsilon=e}\left(u_{V^{\prime}}\right)$ is continuous. The proof for case 2$)$ is analogous with the roles of $U_{V^{\prime}}$ and $U_{B^{\prime}}$ exchanged.

Lemma 4. a) $\frac{\partial}{\partial v} f_{V \mid S=s}(v)$ is continuous on $I_{V, S}$. b) $\frac{\partial}{\partial v} f_{V \mid Q=q}(v)$ is continuous on $I_{V, Q}$.

Proof: We only prove part a), and the proof of part b) is similar. Note that

$$
\begin{aligned}
f_{V \mid S=s, S^{*}=s^{*}}(v) & =\frac{f_{V, U_{V^{\prime}}, U_{B^{\prime}} \mid U=u, \varepsilon=e}\left(v, u_{V^{\prime}}, u_{B^{\prime}}\right)}{f_{U_{V^{\prime}}, U_{B^{\prime}} \mid U=u, \varepsilon=e}\left(u_{V^{\prime}}, u_{B^{\prime}}\right)} \\
f_{V \mid S=s}(v) & =\int \frac{f_{V, U_{V^{\prime}}, U_{B^{\prime}} \mid U=u, \varepsilon=e}\left(v, u_{V^{\prime}}, u_{B^{\prime}}\right)}{f_{U_{V^{\prime}}, U_{B^{\prime}} \mid U=u, \varepsilon=e}\left(u_{V^{\prime}}, u_{B^{\prime}}\right)} f_{S^{*} \mid S=s}\left(s^{*}\right) d s^{*},
\end{aligned}
$$

where the first line follows by Bayes' rule, and we integrate both sides over $f_{S^{*} \mid S=s}\left(s^{*}\right)$ to arrive at the second line. Taking derivatives with respect to $v$ on both sides, interchanging differentiation and integration (permitted by Assumption 4a, Lemma 3 and Roussas (2004)), we obtain the result following Lemma 1.

Lemma 5. a) $\frac{\partial}{\partial v^{*}} f_{V^{*} \mid S=s}\left(v^{*}\right)$ is continuous on $I_{V^{*}, S}$. b) $\frac{\partial}{\partial v^{*}} f_{V^{*} \mid Q=q}\left(v^{*}\right)$ is continuous on $I_{V^{*}, Q}$.

Proof: We only prove part a), and the proof of part b) is similar. Note that after applying Bayes' Rule 
and rearranging, we obtain

$$
\begin{aligned}
f_{V^{*} \mid S=s, S^{*}=s^{*}}\left(v^{*}\right)= & \operatorname{Pr}\left[U_{V}=0 \mid S=s, S^{*}=s^{*}\right] f_{V \mid S=s, S^{*}=s^{*}, U_{V}=0}\left(v^{*}\right) \\
& +\operatorname{Pr}\left[U_{V} \neq 0 \mid S=s, S^{*}=s^{*}\right] f_{V^{*} \mid S=s, S^{*}=s^{*}, U_{V} \neq 0}\left(v^{*}\right) \\
= & \operatorname{Pr}\left[U_{V}=0 \mid S=s, S^{*}=s^{*}\right] \operatorname{Pr}\left[U_{V}=0 \mid V=v^{*}, S=s, S^{*}=s^{*}\right] \frac{f_{V \mid S=s, S^{*}=s^{*}}\left(v^{*}\right)}{\operatorname{Pr}\left[U_{V}=0 \mid S=s, S^{*}=s^{*}\right]} \\
& +\operatorname{Pr}\left[U_{V} \neq 0 \mid S=s, S^{*}=s^{*}\right] \operatorname{Pr}\left[U_{V} \neq 0 \mid V=v^{*}-u_{V^{\prime}}, S=s, S^{*}=s^{*}\right] \frac{f_{V \mid S=s, S^{*}=s^{*}}\left(v^{*}-u_{V^{\prime}}\right)}{\operatorname{Pr}\left[U_{V} \neq 0 \mid S=s, S^{*}=s^{*}\right]} \\
= & \operatorname{Pr}\left[U_{V}=0 \mid V=v^{*}, S=s, S^{*}=s^{*}\right] f_{V \mid S=s, S^{*}=s^{*}}\left(v^{*}\right) \\
& +\operatorname{Pr}\left[U_{V} \neq 0 \mid V=v^{*}-u_{V^{\prime}}, S=s, S^{*}=s^{*}\right] f_{V \mid S=s, S^{*}=s^{*}}\left(v^{*}-u_{V^{\prime}}\right) .
\end{aligned}
$$

Multiplying both sides of the last line by $f_{S^{*} \mid S=s}\left(s^{*}\right)$ and integrating over $s^{*}$, taking the partial derivative with respect to $v^{*}$, and applying Assumptions 4a and 5 and Lemmas 3 and 4, we have the desired result.

Lemma 6. a) $\frac{\partial}{\partial v^{*}} \operatorname{Pr}\left[G_{V}=i, G_{B}=j \mid V^{*}=v^{*}, S=s\right]$ and $\frac{\partial}{\partial v^{*}} \operatorname{Pr}\left[G_{V}=i \mid V^{*}=v^{*}, S=s\right]$ are continuous on the set $\left\{\left(v^{*}, s\right): f_{V^{*} \mid S=s}\left(v^{*}\right)>0\right\}$ for $i, j=0,1$. b) $\frac{\partial}{\partial v^{*}} \operatorname{Pr}\left[G_{V}=i, G_{B}=j \mid V^{*}=v^{*}, Q=q\right]$ and $\frac{\partial}{\partial v^{*}} \operatorname{Pr}\left[G_{V}=\right.$ $\left.i \mid V^{*}=v^{*}, Q=q\right]$ are continuous on the $\operatorname{set}\left\{\left(v^{*}, q\right): f_{V^{*} \mid Q=q}\left(v^{*}\right)>0\right\}$ for $i, j=0,1$.

Proof: Again we only prove part a). First, note that the continuous differentiability of $\operatorname{Pr}\left[G_{V}=i, G_{B}=\right.$ $\left.j \mid V^{*}=v^{*}, S=s\right]$ and $\operatorname{Pr}\left[G_{V}=i \mid V^{*}=v^{*}, S=s\right]$ is only needed on the set $\left\{\left(v^{*}, s\right): f_{V^{*} \mid S=s}\left(v^{*}\right)>0\right\}$ for the purpose of proving Proposition 2, because these quantities are always multiplied by $f_{V^{*} \mid S=s}\left(v^{*}\right)$ when they appear in subsequent proofs. We consider the two cases of $i=0,1$ separately. For case 1 , where $i=0$,

$$
\begin{aligned}
\operatorname{Pr}\left[G_{V}=0, G_{B}=j \mid V^{*}=v^{*}, S=s\right] & =f_{V^{*} \mid S=s, G_{V}=0, G_{B}=j}\left(v^{*}\right) \frac{\operatorname{Pr}\left[G_{V}=0, G_{B}=j \mid S=s\right]}{f_{V^{*} \mid S=s}\left(v^{*}\right)} \\
& =f_{V \mid S=s, G_{V}=0, G_{B}=j}\left(v^{*}\right) \frac{\operatorname{Pr}\left[G_{V}=0, G_{B}=j \mid S=s\right]}{f_{V^{*} \mid S=s}\left(v^{*}\right)} \\
& =f_{V \mid S=s, G_{V}=0, G_{B}=j}\left(v^{*}\right) \frac{\operatorname{Pr}\left[G_{V}=0, G_{B}=j \mid S=s\right]}{f_{V \mid S=s}\left(v^{*}\right)} \frac{f_{V \mid S=s}\left(v^{*}\right)}{f_{V^{*} \mid S=s}\left(v^{*}\right)} \\
& =\operatorname{Pr}\left[G_{V}=0, G_{B}=j \mid V=v^{*}, S=s\right] \frac{f_{V \mid S=s}\left(v^{*}\right)}{f_{V^{*} \mid S=s}\left(v^{*}\right)} \\
& =\int \pi^{0 j}\left(v^{*}, u, \varepsilon, u_{V^{\prime}}, u_{B^{\prime}}\right) f_{S^{*} \mid V=v^{*}, S=s}\left(s^{*}\right) d s^{*} \frac{f_{V \mid S=s}\left(v^{*}\right)}{f_{V^{*} \mid S=s}\left(v^{*}\right)} \\
& =\int \pi^{0 j}\left(v^{*}, u, \varepsilon, u_{V^{\prime}}, u_{B^{\prime}}\right) f_{V \mid S^{*}=s^{*}, S=s}\left(v^{*}\right) \frac{f_{S^{*} \mid S=s}\left(s^{*}\right)}{f_{V \mid S=s}\left(v^{*}\right)} d s^{*} \frac{f_{V \mid S=s}\left(v^{*}\right)}{f_{V^{*} \mid S=s}\left(v^{*}\right)}
\end{aligned}
$$

The partial derivative of the right hand side w.r.t. $v^{*}$ in the last line is continuous on $I_{V^{*}, S}$ by Assumption 5 and Lemmas 3, 4 and 5. For case 2 where $i=1$, 


$$
\begin{aligned}
& \operatorname{Pr}\left[G_{V}=1, G_{B}=j \mid V^{*}=v^{*}, S=s\right] \\
= & \int \operatorname{Pr}\left[G_{V}=1, G_{B}=j \mid V^{*}=v^{*}, S=s, S^{*}=s^{*}\right] f_{S^{*} \mid V^{*}=v^{*}, S=s}\left(s^{*}\right) d s^{*} \\
= & \int \operatorname{Pr}\left[G_{V}=1, G_{B}=j \mid V=v^{*}-u_{V^{\prime}}, S=s, S^{*}=s^{*}\right] \frac{f_{V^{*} \mid S=s, S^{*}=s^{*}}\left(v^{*}\right) f_{S^{*} \mid S=s}\left(s^{*}\right)}{f_{V^{*} \mid S=s}\left(v^{*}\right)} d s^{*} \\
= & \int \pi^{1 j}\left(v^{*}-u_{V^{\prime}}, u, \varepsilon, u_{V^{\prime}}, u_{B^{\prime}}\right) \frac{f_{V^{*} \mid S=s, S^{*}=s^{*}}\left(v^{*}\right) f_{S^{*} \mid S=s}\left(s^{*}\right)}{f_{V^{*} \mid S=s}\left(v^{*}\right)} d s^{*} .
\end{aligned}
$$

Its partial derivative w.r.t. $v^{*}$ is continuous on $I_{V^{*}, S}$ for the same reason as in case 1 .

Since $\operatorname{Pr}\left[G_{V}=i \mid V^{*}=v^{*}, S=s\right]=\sum_{j} \operatorname{Pr}\left[G_{V}=i, G_{B}=j \mid V^{*}=v^{*}, S=s\right]$, the continuous differentiability with respect to $v^{*}$ of $\operatorname{Pr}\left[G_{V}=i, G_{B}=j \mid V^{*}=v^{*}, S=s\right]$ implies that of $\operatorname{Pr}\left[G_{V}=i \mid V^{*}=v^{*}, S=s\right]$.

\section{Proof of Proposition 2}

For part (a), the proof is the same as for part (a) in Proposition 1, replacing $V$ with $V^{*}$, letting the pair $(U, \varepsilon)$ serve the role of $U$ and using Lemma 5.

For part (b), we can write

$$
\begin{aligned}
E\left[Y \mid V^{*}=v^{*}\right]= & \int E\left[Y \mid V^{*}=v^{*}, U=u, \varepsilon=e\right] d F_{U, \varepsilon \mid V^{*}=v^{*}}(u, e) \\
= & \int\left(E\left[Y \mid U_{V}=0, V^{*}=v^{*}, U=u, \varepsilon=e\right] \operatorname{Pr}\left[U_{V}=0 \mid V^{*}=v^{*}, U=u, \varepsilon=e\right]+\right. \\
& \left.E\left[Y \mid U_{V} \neq 0, V^{*}=v^{*}, U=u, \varepsilon=e\right] \operatorname{Pr}\left[U_{V} \neq 0 \mid V^{*}=v^{*}, U=u, \varepsilon=e\right]\right) d F_{U, \varepsilon \mid V^{*}=v^{*}}(u, e) \\
= & \int\left(z_{1} z_{2}+\left[\int z_{3} z_{4} d u_{V^{\prime}}\right] \cdot\left[1-z_{2}\right]\right) z_{5} d F_{U, \varepsilon}(u, e),
\end{aligned}
$$

where the second line follows from the law of iterated expectations, and to ease exposition below, we use the notation:

$$
\begin{aligned}
z_{1} & \equiv y\left(b\left(v^{*}, e\right), v^{*}, u\right) \\
z_{2} & \equiv \operatorname{Pr}\left[V=V^{*} \mid V^{*}=v^{*}, U=u, \varepsilon=e\right] \\
z_{3} & \equiv y\left(b\left(v^{*}-u_{V^{\prime}}, e\right), v^{*}-u_{V^{\prime}}, u\right) \\
z_{4} & \equiv f_{U_{V^{\prime}} \mid U_{V} \neq 0, V^{*}=v^{*}, U=u, \varepsilon=e}\left(u_{V^{\prime}}\right) \\
z_{5} & \equiv \frac{f_{V^{*} \mid U=u, \varepsilon=e}\left(v^{*}\right)}{f_{V^{*}}\left(v^{*}\right)} .
\end{aligned}
$$

The derivative of $E\left[Y \mid V^{*}=v^{*}\right]$ in equation (11) with respect to $v^{*}$ is

$$
\begin{aligned}
\frac{d E\left[Y \mid V^{*}=v^{*}\right]}{d v^{*}}= & \int z_{1}^{\prime} z_{2} z_{5} d F_{U, \varepsilon}(u, e)+\int z_{1} \frac{\partial\left(z_{2} z_{5}\right)}{\partial v^{*}} d F_{U, \varepsilon}(u, e) \\
& +\int \frac{\partial\left[\left(\int z_{3} z_{4} d u_{V^{\prime}}\right)\left[1-z_{2}\right] z_{5}\right]}{\partial v^{*}} d F_{U, \varepsilon}(u, e),
\end{aligned}
$$

where $z_{j}^{\prime}$ denotes the partial derivative of $z_{j}$ with respect to $v^{*}$, provided that the integrands are continuous. 
In a parallel fashion, we can write

$$
\begin{aligned}
E\left[B^{*} \mid V^{*}=v^{*}\right]= & \int\left\{\left[z_{6}+z_{8}\left(1-z_{7}\right)\right] z_{13}\right. \\
& \left.+\left[\left(\int z_{9} z_{10} d u_{V^{\prime}}\right) z_{11}+\left(\int\left(z_{9}+u_{B^{\prime}}\right) z_{12} d u_{V^{\prime}} d u_{B^{\prime}}\right)\left(1-z_{11}\right)\right]\left(1-z_{13}\right)\right\} z_{14} d F_{\varepsilon}(e),
\end{aligned}
$$

with

$$
\begin{aligned}
z_{6} & \equiv b\left(v^{*}, e\right) \\
z_{7} & \equiv \operatorname{Pr}\left[U_{B}=0 \mid U_{V}=0, V^{*}=v^{*}, \varepsilon=e\right] \\
z_{8} & \equiv \int u_{B^{\prime}} f_{U_{B^{\prime}} \mid U_{V}=0, U_{B} \neq 0, V^{*}=v^{*}, \varepsilon=e}\left(u_{B^{\prime}}\right) d u_{B^{\prime}} \\
z_{9} & \equiv b\left(v^{*}-u_{V^{\prime}}, e\right) \\
z_{10} & \equiv f_{U_{V^{\prime}} \mid U_{B}=0, U_{V} \neq 0, V^{*}=v^{*}, \varepsilon=e}\left(u_{V^{\prime}}\right) \\
z_{11} & \equiv \operatorname{Pr}\left[U_{B}=0 \mid U_{V} \neq 0, V^{*}=v^{*}, \varepsilon=e\right] \\
z_{12} & \equiv f_{U_{V^{\prime}}, U_{B^{\prime}} \mid U_{V} \neq 0, U_{B} \neq 0, V^{*}=v^{*}, \varepsilon=e}\left(u_{V^{\prime}}, u_{B^{\prime}}\right) \\
z_{13} & \equiv \operatorname{Pr}\left[V=V^{*} \mid V^{*}=v^{*}, \varepsilon=e\right] \\
z_{14} & \equiv \frac{f_{V^{*} \mid \varepsilon=e}\left(v^{*}\right)}{f_{V^{*}}\left(v^{*}\right)} .
\end{aligned}
$$

And the analogous derivative with respect to $v^{*}$ is

$$
\begin{aligned}
\frac{d E\left[B^{*} \mid V^{*}=v^{*}\right]}{d v^{*}}= & \int z_{6}^{\prime} z_{13} z_{14} d F_{\varepsilon}(e)+\int z_{6} \frac{\partial}{\partial v^{*}}\left(z_{13} z_{14}\right) d F_{\varepsilon}(e) \\
& +\frac{\partial}{\partial v^{*}} \int\left[z_{8}\left(1-z_{7}\right) z_{13} z_{14}\right] d F_{\varepsilon}(e) \\
& +\frac{\partial}{\partial v^{*}} \int\left\{\int z_{9} z_{10} d u_{V^{\prime}} \cdot z_{11}+\right. \\
& \left.\left.\iint\left(z_{9}+u_{B^{\prime}}\right) z_{12} d u_{V^{\prime}} d u_{B^{\prime}} \cdot\left(1-z_{11}\right)\right\}\left(1-z_{13} z_{14}\right)\right\} d F_{\varepsilon}(e),
\end{aligned}
$$

provided that the integrands are continuous.

The proof of part (b) follows from showing that the partial derivatives of $z_{2}, \int z_{3} z_{4} d u_{V^{\prime}}, z_{5}, z_{7}, z_{8}$, $\int z_{9} z_{10} d u_{V^{\prime}},, z_{11}, \iint\left(z_{9}+u_{B^{\prime}}\right) z_{12} d u_{V^{\prime}} d u_{B^{\prime}}$ and $z_{13} z_{14}$, with respect to $v^{*}$ are continuous, and noting that $z_{1}$ and $z_{6}$ are continuous by Assumptions 1a, 2, and 3a. From this it follows that there is no discontinuity in all but the first term on the right hand side of (12) and (13) at $v^{*}=0$ and that the RKD estimand is the ratio of the discontinuities in the first terms of those two equations.

As shown by Lemma $6, z_{2}$ is continuously differentiable in $v^{*}$. 
$z_{4}$ is continuously differentiable in $v^{*}$ because

$$
\begin{aligned}
f_{U_{V^{\prime}} \mid U_{V} \neq 0, V^{*}=v^{*}, U=u, \varepsilon=e}\left(u_{V^{\prime}}\right) & =\frac{\operatorname{Pr}\left[U_{V} \neq 0 \mid U_{V^{\prime}}=u_{V^{\prime}}, V^{*}=v^{*}, U=u, \varepsilon=e\right] f_{U_{V^{\prime}} \mid V^{*}=v^{*}, U=u, \varepsilon=e}\left(u_{V^{\prime}}\right)}{\operatorname{Pr}\left[U_{V} \neq 0 \mid V^{*}=v^{*}, U=u, \varepsilon=e\right]} \\
& =\frac{\left(1-\operatorname{Pr}\left[U_{V}=0 \mid U_{V^{\prime}}=u_{V^{\prime}}, V^{*}=v^{*}, U=u, \varepsilon=e\right]\right) \frac{f_{V^{*} \mid U_{V^{\prime}}=u_{V^{\prime}}, U=u, \varepsilon=e}\left(v^{*}\right) f_{V^{\prime}} \mid U=u, \varepsilon=e}{f_{V^{*}} \mid U=u, \varepsilon=e}\left(u_{V^{\prime}}\right)}{1-z_{2}}
\end{aligned}
$$

and the derivative of the last line is continuous by Lemmas 3,5 and 6 .

We break up the integral $\int z_{3} z_{4} d u_{V^{\prime}}$ into two pieces

$$
\begin{aligned}
\int z_{3} z_{4} d u_{V^{\prime}}= & \int_{c_{V_{V^{\prime}}}}^{v^{*}} z_{3} z_{4} d u_{V^{\prime}}+\int_{v^{*}}^{d_{V_{V^{\prime}}}} z_{3} z_{4} d u_{V^{\prime}} \\
= & \int_{c_{V_{V^{\prime}}}^{v^{*}}}^{d_{V^{\prime}}} y\left(b^{+}\left(v^{*}-u_{V^{\prime}}, e\right), v^{*}-u_{V^{\prime}}, u\right) z_{4} d u_{V^{\prime}} \\
& +\int_{v^{*}}^{d_{V^{\prime}}} y\left(b^{-}\left(v^{*}-u_{V^{\prime}}, e\right), v^{*}-u_{V^{\prime}}, u\right) z_{4} d u_{V^{\prime}},
\end{aligned}
$$

where $c_{U_{V^{\prime}}}$ and $d_{U_{V^{\prime}}}$ are the lower and upper end point of the support of $U_{V^{\prime}}, b^{+}(v, e)=b(v, e)$ for $v \geqslant 0$ and all $e$ and $b^{-}(v, e)=b(v, e)$ for $v \leqslant 0$ and all $e$. Denote $y\left(b^{ \pm}\left(v^{*}-u_{V^{\prime}}, e\right), v^{*}-u_{V^{\prime}}, u\right)$ by $z_{3}^{ \pm}$. Note that $z_{3}^{+}$ and $z_{3}^{-}$are continuously differentiable in $v^{*}$ on $\left[c_{U_{V^{\prime}}}, v^{*}\right]$ and $\left[v^{*}, d_{U_{V^{\prime}}}\right]$ respectively by Assumptions 1a, 2 and $3 \mathrm{a}$, where $c_{U_{V^{\prime}}}$ and $d_{U_{V^{\prime}}}$ are the lower and upper endpoints of the support $I_{U_{V}^{\prime}}$. Since $z_{4}$ is also continuously differentiable as shown above, we can apply the Newton-Leibniz formula, which yields

$$
\begin{aligned}
\frac{\partial}{\partial v^{*}}\left(\int_{c_{V_{V^{\prime}}}^{v^{*}}}^{v^{*}} z_{3}^{+} z_{4} d u_{V^{\prime}}+\int_{v^{*}}^{d_{V_{V^{\prime}}}} z_{3}^{-} z_{4} d u_{V^{\prime}}\right)= & \int_{c_{V_{V^{\prime}}}^{v^{*}}}^{v^{*}} \frac{\partial}{\partial v^{*}}\left(z_{3}^{+} z_{4}\right) d u_{V^{\prime}}+\int_{v^{*}}^{d_{V_{V^{\prime}}}} \frac{\partial}{\partial v^{*}}\left(z_{3}^{-} z_{4}\right) d u_{V^{\prime}} \\
& +\left.z_{3}^{+} z_{4}\right|_{u_{V^{\prime}}}=v^{*}-\left.z_{3}^{-} z_{4}\right|_{u_{V^{\prime}}}=v^{*} .
\end{aligned}
$$

By Assumptions 1a and 3a, $z_{3}$ is continuous, and it follows that $\left.z_{3}^{+} z_{4}\right|_{u_{V^{\prime}}=v^{*}}-\left.z_{3}^{-} z_{4}\right|_{u_{V^{\prime}}=v^{*}}=0$. Since $\int_{c_{V_{V^{\prime}}}^{v^{*}}}^{v^{*}} \frac{\partial}{\partial v^{*}}\left(z_{3}^{+} z_{4}\right) d u_{V^{\prime}}$ and $\int_{v^{*}}^{d_{U_{V^{\prime}}}} \frac{\partial}{\partial v^{*}}\left(z_{3}^{-} z_{4}\right) d u_{V^{\prime}}$ are continuous, $\int z_{3} z_{4} d u_{V^{\prime}}$ is continuously differentiable in $v^{*}$.

$z_{5}$ is continuously differentiable in $v^{*}$ by Lemma 5 and Assumption 3a-note that the continuous differentiability of $f_{V^{*}}\left(v^{*}\right)=\int f_{V^{*} \mid U=u, \varepsilon=e} d F_{U, \varepsilon}(u, e)$ is a part of Corollary 1, and it follows directly from Lemma 5; $z_{6}$ is continuously differentiable by Assumption $3 \mathrm{a}$, and $z_{7}$ is continuously differentiable in $v^{*}$ because

$$
\operatorname{Pr}\left[U_{B}=0 \mid U_{V}=0, V^{*}=v^{*}, \varepsilon=e\right]=\frac{\operatorname{Pr}\left[U_{V}=0, U_{B}=0 \mid V^{*}=v^{*}, \varepsilon=e\right]}{\operatorname{Pr}\left[U_{V}=0 \mid V=v^{*}, \varepsilon=e\right]},
$$

where the derivative of the right hand side is continuous in $v^{*}$ by Lemma 6 . 
$z_{8}$ is continuously differentiable in $v^{*}$ because

$$
\begin{aligned}
& \int u_{B^{\prime}} f_{U_{B^{\prime}} \mid U_{V}=0, U_{B} \neq 0, V^{*}=v^{*}, \varepsilon=e}\left(u_{B^{\prime}}\right) d u_{B^{\prime}} \\
= & \int u_{B^{\prime}} \operatorname{Pr}\left[U_{V}=0 \mid U_{B^{\prime}}=u_{B^{\prime}}, V^{*}=v^{*}, \varepsilon=e\right] \frac{f_{U_{B^{\prime}} \mid V^{*}=v^{*}, \varepsilon=e}\left(u_{B^{\prime}}\right)}{\operatorname{Pr}\left[U_{V}=0 \mid V^{*}=v^{*}, \varepsilon=e\right]} d u_{B^{\prime}} \\
= & \int u_{B^{\prime}} \operatorname{Pr}\left[U_{V}=0 \mid U_{B^{\prime}}=u_{B^{\prime}}, V^{*}=v^{*}, \varepsilon=e\right] \frac{f_{V^{*} \mid U_{B^{\prime}}=u_{B^{\prime}}, \varepsilon=e}\left(v^{*}\right) f_{U_{B^{\prime}} \mid \varepsilon=e}\left(u_{B^{\prime}}\right)}{f_{V^{*} \mid \varepsilon=e}\left(v^{*}\right) \operatorname{Pr}\left[U_{V}=0 \mid V=v^{*}, \varepsilon=e\right]} d u_{B^{\prime}},
\end{aligned}
$$

where the continuous differentiability of the last line in $v^{*}$ is implied by Lemmas 3,5 and 6. By a similar application of the Bayes' Rule, we can show that $z_{10}$ is continuously differentiable in $v^{*}$. Consequently, $\int z_{9} z_{10} d u_{V^{\prime}}$ is continuously differentiable in $v^{*}$ by applying the same argument used for $\int z_{3} z_{4} d u_{V^{\prime}}$.

The quantity $z_{11}$ is continuously differentiable in $v^{*}$ because of Lemma 6 and

$$
z_{11}=\frac{\operatorname{Pr}\left[U_{B}=0, U_{V} \neq 0 \mid V^{*}=v^{*}, \varepsilon=e\right]}{\operatorname{Pr}\left[U_{V} \neq 0 \mid V^{*}=v^{*}, \varepsilon=e\right]}
$$

$z_{12}$ can be expressed as

$$
\begin{aligned}
& f_{U_{V^{\prime}}, U_{B^{\prime}} \mid U_{V} \neq 0, U_{B} \neq 0, V^{*}=v^{*}, \varepsilon=e}\left(u_{V^{\prime}}, u_{B^{\prime}}\right) \\
& =\frac{\operatorname{Pr}\left[U_{V} \neq 0, U_{B} \neq 0 \mid U_{V^{\prime}}=u_{V^{\prime}}, U_{B^{\prime}}=u_{B^{\prime}}, V^{*}=v^{*}, \varepsilon=e\right]}{\operatorname{Pr}\left[U_{V} \neq 0, U_{B} \neq 0 \mid V^{*}=v^{*}, \varepsilon=e\right]} f_{U_{V^{\prime}}, U_{B^{\prime}} \mid V^{*}=v^{*}, \varepsilon=e}\left(u_{V^{\prime}}, u_{B^{\prime}}\right) \\
& =\frac{\operatorname{Pr}\left[U_{V} \neq 0, U_{B} \neq 0 \mid U_{V^{\prime}}=u_{V^{\prime}}, U_{B^{\prime}}=u_{B^{\prime}}, V^{*}=v^{*}, \varepsilon=e\right]}{\operatorname{Pr}\left[U_{V} \neq 0, U_{B} \neq 0 \mid V^{*}=v^{*}, \varepsilon=e\right]} . \\
& \frac{f_{V^{*} \mid U_{V^{\prime}}=u_{V^{\prime}}, U_{B^{\prime}}=u_{B^{\prime}}, \varepsilon=e}\left(v^{*}\right)}{f_{V^{*} \mid \varepsilon=e}\left(v^{*}\right)} f_{U_{V^{\prime}}, U_{B^{\prime}} \mid \varepsilon=e}\left(u_{V^{\prime}}, u_{B^{\prime}}\right),
\end{aligned}
$$

and $z_{12}$ is continuously differentiable by Lemmas 3,5 and 6 . It follows that $\iint\left(z_{9}+u_{B^{\prime}}\right) z_{12} d u_{V^{\prime}} d u_{B^{\prime}}$ is continuously differentiable by the same argument as that for $\int z_{3} z_{4} d u_{V^{\prime}}$.Finally, $z_{13}$ is continuously differentiable in $v^{*}$ by Lemma 6 and $z_{14}$ by Lemma 5 and Assumption 3a.

As a result of the smoothness of the above terms along with Theorem 5 on p. 97 of Roussas (2004), we can write

$$
\begin{aligned}
& \left.\lim _{v_{0} \rightarrow 0^{+}} \frac{d E\left[Y \mid V^{*}=v^{*}\right]}{d v^{*}}\right|_{v^{*}=v_{0}}-\left.\lim _{v_{0} \rightarrow 0^{-}} \frac{d E\left[Y \mid V^{*}=v^{*}\right]}{d v^{*}}\right|_{v^{*}=v_{0}} \\
= & \lim _{v_{0} \rightarrow 0^{+}} \int z_{1}^{\prime} z_{2} z_{5} d F_{U, \varepsilon}(u, e)-\lim _{v_{0} \rightarrow 0^{-}} \int z_{1}^{\prime} z_{2} z_{5} d F_{U, \varepsilon}(u, e) \\
= & \left.\int\left(\lim _{v_{0} \rightarrow 0^{+}} z_{1}^{\prime}-\lim _{v_{0} \rightarrow 0^{-}} z_{1}^{\prime}\right) z_{2} z_{5}\right|_{v^{*}=v_{0}} d F_{U, \varepsilon}(u, e) \\
= & \left.\int y_{1}(b(0, e), 0, u)\left(b_{1}^{+}(e)-b_{1}^{-}(e)\right) z_{2} z_{5}\right|_{v^{*}=v_{0}} d F_{U, \varepsilon}(u, e) .
\end{aligned}
$$

The interchange of limit of integration is allowed by the dominated convergence theorem since $z_{1}^{\prime} z_{2} z_{5}$ is 
continuous over a compact rectangle. The last line follows from Assumptions 1a and 3a.

Similarly, we can write

$$
\begin{aligned}
& \left.\lim _{v_{0} \rightarrow 0^{+}} \frac{d E\left[B^{*} \mid V^{*}=v^{*}\right]}{d v^{*}}\right|_{v^{*}=v_{0}}-\left.\lim _{v_{0} \rightarrow 0^{-}} \frac{d E\left[B^{*} \mid V^{*}=v^{*}\right]}{d v^{*}}\right|_{v^{*}=v_{0}} \\
= & \lim _{v_{0} \rightarrow 0^{+}} \int z_{6}^{\prime} z_{13} z_{14} d F_{\varepsilon}(e)-\lim _{v_{0} \rightarrow 0^{-}} \int z_{6}^{\prime} z_{13} z_{14} d F_{\varepsilon}(e) \\
= & \left.\int\left(\lim _{v_{0} \rightarrow 0^{+}} z_{6}^{\prime}-\lim _{v_{0} \rightarrow 0^{-}} z_{6}^{\prime}\right) z_{13} z_{14}\right|_{v^{*}=v_{0}} d F_{\varepsilon}(e) \\
= & \left.\int\left(b_{1}^{+}(e)-b_{1}^{-}(e)\right) z_{13} z_{14}\right|_{v^{*}=v_{0}} d F_{\varepsilon}(e) .
\end{aligned}
$$

Finally, consider the term $\left.z_{2} z_{5}\right|_{v^{*}=v_{0}}$. First, a similar argument as in 6 leads to

$$
z_{2}=\operatorname{Pr}\left[V=V^{*} \mid V=v^{*}, U=u, \varepsilon=e\right] \frac{f_{V \mid U=u, \varepsilon=e}\left(v^{*}\right)}{f_{V^{*} \mid U=u, \varepsilon=e}\left(v^{*}\right)} .
$$

After applying Bayes' Rule and rearranging, we have

$$
\begin{aligned}
\left.z_{2} z_{5}\right|_{v^{*}=v_{0}} & =\operatorname{Pr}\left[V=V^{*} \mid V=0, U=u, \varepsilon=e\right] \frac{f_{V \mid U=u, \varepsilon=e}(0)}{f_{V^{*} \mid U=u, \varepsilon=e}(0)} \frac{f_{V^{*} \mid U=u, \varepsilon=e}(0)}{f_{V^{*}}(0)} \\
& =\operatorname{Pr}\left[V=V^{*} \mid V=0, U=u, \varepsilon=e\right] \frac{f_{V \mid U=u, \varepsilon=e}(0)}{f_{V}(0)} \frac{f_{V}(0)}{f_{V^{*}}(0)} .
\end{aligned}
$$

Similarly, we can derive

$$
\left.z_{13} z_{14}\right|_{v^{*}=v_{0}}=\operatorname{Pr}\left[V=V^{*} \mid V=0, \varepsilon=e\right] \frac{f_{V \mid \varepsilon=e}(0)}{f_{V}(0)} \frac{f_{V}(0)}{f_{V^{*}}(0)} .
$$

Because $\frac{f_{V}(0)}{f_{V^{*}}(0)}$ can be pulled out of the integral in both (14) and (15), we have the result

$$
\frac{\left.\lim _{v_{0} \rightarrow 0^{+}} \frac{d E\left[Y \mid V^{*}=v^{*}\right]}{d v^{*}}\right|_{v^{*}=v_{0}}-\left.\lim _{v_{0} \rightarrow 0^{-}} \frac{d E\left[Y \mid V^{*}=v^{*}\right]}{d v^{*}}\right|_{v^{*}=v_{0}}}{\left.\lim _{v_{0} \rightarrow 0^{+}} \frac{d E\left[B^{*} \mid V^{*}=v^{*}\right]}{d v^{*}}\right|_{v^{*}=v_{0}}-\left.\lim _{v_{0} \rightarrow 0^{-}} \frac{d E\left[B^{*} \mid V^{*}=v^{*}\right]}{d v^{*}}\right|_{v^{*}=v_{0}}}=\int y_{1}(b(0, e), 0, u) \varphi(u, e) d F_{U, \varepsilon}(u, e),
$$

where $\varphi(u, e)=\frac{\operatorname{Pr}\left[U_{V}=0 \mid V=0, U=u, \varepsilon=e\right]\left(b_{1}^{+}(e)-b_{1}^{-}(e)\right) \frac{f_{V \mid U=u, \varepsilon=e^{(0)}}}{f_{V}(0)}}{\int \operatorname{Pr}\left[U_{V}=0 \mid V=0, \varepsilon=\omega\right]\left(b_{1}^{+}(\omega)-b_{1}^{-}(\omega)\right) \frac{f_{V \mid \varepsilon=\omega^{(0)}}}{f_{V}(0)} d F_{\varepsilon}(\omega)}$.

Note that Assumptions 3a and 6 guarantee non-negative, finite weights and that $\int \varphi(u, e) d F_{U, \varepsilon}(u, e)=1$.

\section{A.2 Identification in the Presence of Both Slope and Level Changes-Remark 3}

In Remark 3, we consider the identification of the treatment effect when there is both a level change and a slope change at the threshold $V=0$. To ease exposition, define $\lim _{v_{0} \rightarrow 0^{+}} b^{\prime}\left(v_{0}\right)=b^{\prime}\left(0^{+}\right), \lim _{v_{0} \rightarrow 0^{-}} b^{\prime}\left(v_{0}\right)=b^{\prime}\left(0^{-}\right)$, $\lim _{v_{0} \rightarrow 0^{+}} b\left(v_{0}\right)=b\left(0^{+}\right)$and $\lim _{v_{0} \rightarrow 0^{-}} b\left(v_{0}\right)=b\left(0^{-}\right)$. We study the case where $b^{\prime}\left(0^{+}\right) \neq b^{\prime}\left(0^{-}\right)$and $b\left(0^{+}\right) \neq b\left(0^{-}\right)$, but $b()$ is still a smooth function on $I_{V} /\{0\}$. Similar to the derivation in the proof of Proposition 1, we can show that the RK estimand identifies the following parameter: 


$$
\begin{aligned}
\frac{\left.\lim _{v_{0} \rightarrow 0^{+}} \frac{d E[Y \mid V=v]}{d v}\right|_{v=v_{0}}-\left.\lim _{v_{0} \rightarrow 0^{-}} \frac{d E[Y \mid V=v]}{d v}\right|_{v=v_{0}}}{\left.\lim _{v_{0} \rightarrow 0^{+}} \frac{d b(v)}{d v}\right|_{v=v_{0}}-\left.\lim _{v_{0} \rightarrow 0^{-}} \frac{d b(v)}{d v}\right|_{v=v_{0}}}= & \frac{b^{\prime}\left(0^{+}\right) \int y_{1}\left(b\left(0^{+}\right), 0, u\right) \frac{f_{V \mid U=u}(0)}{f_{V}(0)} d F_{U}(u)-b^{\prime}\left(0^{-}\right) \int y_{1}\left(b\left(0^{-}\right), 0, u\right) \frac{f_{V \mid U=u}(0)}{f_{V}(0)} d F_{U}(u)}{b^{\prime}\left(0^{+}\right)-b^{\prime}\left(0^{-}\right)} \\
& +\frac{\int\left\{\left[y_{2}\left(b\left(0^{+}\right), 0, u\right)-y_{2}\left(b\left(0^{-}\right), 0, u\right)\right] \frac{f_{V \mid U=u}(0)}{f_{V}(0)}+\left[y\left(b\left(0^{+}\right), 0, u\right)-y\left(b\left(0^{-}\right), 0, u\right)\right] \frac{\partial}{\partial v} \frac{f_{V \mid U=u}(0)}{f_{V}(0)}\right\} d F_{U}(u)}{b^{\prime}\left(0^{+}\right)-b^{\prime}\left(0^{-}\right)},
\end{aligned}
$$

which is in general not readily interpretable as a weighted average of the causal effect.

On the other hand, we can show that the RD estimand identifies a weighted average of the causal effect of interest:

$$
\begin{aligned}
\frac{\lim _{v_{0} \rightarrow 0^{+}} E\left[Y \mid V=v_{0}\right]-\lim _{v_{0} \rightarrow 0^{-}} E\left[Y \mid V=v_{0}\right]}{\lim _{v_{0} \rightarrow 0^{+}} b\left(v_{0}\right)-\lim _{v_{0} \rightarrow 0^{-}} b\left(v_{0}\right)} & =\frac{\lim _{v_{0} \rightarrow 0^{+}} E\left[y\left(b\left(v_{0}\right), v_{0}, U\right) \mid V=v_{0}\right]-\lim _{v_{0} \rightarrow 0^{-}} E\left[y\left(b\left(v_{0}\right), v_{0}, U\right) \mid V=v_{0}\right]}{\lim _{v_{0} \rightarrow 0^{+}} b\left(v_{0}\right)-\lim _{v_{0} \rightarrow 0^{-}} b\left(v_{0}\right)} \\
& =E\left[\frac{y\left(b\left(0^{+}\right), 0, U\right)-y\left(b\left(0^{-}\right), 0, U\right)}{b\left(0^{+}\right)-b\left(0^{-}\right)} \mid V=0\right] \\
& =E\left[y_{1}(\tilde{b}, 0, U) \mid V=0\right],
\end{aligned}
$$

where $\tilde{b}$ is between $b\left(0^{+}\right)$and $b\left(0^{-}\right)$and the last line follows from the mean value theorem.

Similarly, in the fuzzy framework of section 2.2.2, it can be shown that the RK estimand no longer identifies the causal effect of interest if we allow a discontinuity in $b(\cdot, e)$ for some $e$ at the threshold. However, the RD estimand still identifies a weighted average of the causal effect $y_{1}$. To see this, let $\lim _{v_{0}^{*} \rightarrow 0^{+}} b\left(v_{0}^{*}, e\right) \equiv b\left(0,{ }^{+} e\right), \lim _{v_{0}^{*} \rightarrow 0^{-}} b\left(v_{0}^{*}, e\right) \equiv b\left(0^{-}, e\right)$ and modify Assumption 3 a and Assumption 6 by replacing $b_{1}^{ \pm}(e)$ with $b\left(0^{ \pm}, e\right)$; using notations from the proof of Proposition 2, we have

$$
\begin{aligned}
& \lim _{v_{0} \rightarrow 0^{+}} E\left[Y \mid V^{*}=v_{0}\right]-\lim _{v_{0} \rightarrow 0^{-}} E\left[Y \mid V^{*}=v_{0}\right] \\
& \lim _{v_{0} \rightarrow 0^{+}} E\left[B^{*} \mid V^{*}=v_{0}\right]-\lim _{v_{0} \rightarrow 0^{-}} E\left[B^{*} \mid V^{*}=v_{0}\right]=\frac{\lim _{v_{0} \rightarrow 0^{+}} \int z_{1} z_{2} z_{5} d F_{U, \varepsilon}(u, e)-\lim _{v_{0} \rightarrow 0^{-}} \int z_{1} z_{2} z_{5} d F_{U, \varepsilon}(u, e)}{\lim _{v_{0} \rightarrow 0^{+}} \int z_{6} z_{13} z_{14} d F_{U, \varepsilon}(u, e)-\lim _{v_{0} \rightarrow 0^{-}} \int z_{6} z_{13} z_{14} d F_{U, \varepsilon}(u, e)} \\
&=\frac{\int\left[y\left(b\left(0^{+}, e\right), 0, u\right)-y\left(b\left(0^{-}, e\right), 0, u\right)\right] \operatorname{Pr}\left[U_{V}=0 \mid V=0, U=u, \varepsilon=e\right] \frac{f_{V \mid U=u, \varepsilon=e}(0)}{f_{V}(0)} d F_{U, \varepsilon}(u, e)}{\int\left[b\left(0^{+}, e\right)-b\left(0^{-}, e\right)\right] \operatorname{Pr}\left[U_{V}=0 \mid V=0, \varepsilon=e\right] \frac{f_{V \mid \varepsilon=e}(0)}{f_{V}(0)} d F_{\varepsilon}(e)} \\
&=\frac{\int \frac{y\left(b\left(0^{+}, e\right), 0, u\right)-y\left(b\left(0^{-}, e\right), 0, u\right)}{b\left(0^{+}, e\right)-b\left(0^{-}, e\right)}\left[b\left(0^{+}, e\right)-b\left(0^{-}, e\right)\right] \operatorname{Pr}\left[U_{V}=0 \mid V=0, U=u, \varepsilon=e\right] \frac{f_{V \mid U=u, \varepsilon=e}(0)}{f_{V}(0)} d F_{U, \varepsilon}(u, e)}{\int\left[b\left(0^{+}, e\right)-b\left(0^{-}, e\right)\right] \operatorname{Pr}\left[U_{V}=0 \mid V=0, \varepsilon=e\right] \frac{f_{V \mid \varepsilon=e}(0)}{f_{V}(0)} d F_{\varepsilon}(e)} \\
&=\int y(\tilde{b}(e), 0, u) \psi(e, u) d F_{U, \varepsilon}(u, e),
\end{aligned}
$$

where $\tilde{b}(e)$ is a value between $b\left(0^{+}, e\right)$ and $b\left(0^{-}, e\right)$ for each $e$ and $\psi(e, u)=\frac{\left[b\left(0^{+}, e\right)-b\left(0^{-}, e\right)\right] \operatorname{Pr}\left[U_{V}=0 \mid V=0, U=u, \varepsilon=e\right] \frac{f_{V \mid U=u, \varepsilon=e}(0)}{f_{V}(0)}}{\int\left[b\left(0^{+}, e\right)-b\left(0^{-}, e\right)\right] \operatorname{Pr}\left[U_{V}=0 \mid V=0, \varepsilon=e\right] \frac{f_{V \mid \varepsilon=e}(0)}{f_{V}(0)} d F_{\varepsilon}(e)}$. 


\section{A.3 Applying RKD When the Treatment Variable is Binary-Remark 6}

We provide details on the RK identification result stated in Remark 6. The identifying assumptions are:

Assumption 1c. (Regularity) (i) The support of $U$ and $\eta$ are bounded: they are subsets of the arbitrarily large compact set $I_{U} \subset \mathbb{R}^{m}$ and $I_{\eta}=\left[c_{\eta}, d_{\eta}\right] \subset \mathbb{R}$ respectively. (ii) $y(t, v, u)$ is continuous on $I_{V, U}$ for $t=0,1$. (iii) $t(b, v, n)$ is continuously differentiable on $I_{b(V), V, \eta}$ and is strictly increasing in $n$ for all $b, v \in I_{b(V), V}$.

By Assumption 1c and the implicit function theorem, we can define the continuously differentiable function $\tilde{\eta}: I_{b(V)} \times I_{V} \rightarrow \mathbb{R}$ such that $t(b, v, \tilde{\eta}(b, v))=0$. Let $\tilde{\eta}(b(V), V)$ be the image of $I_{b(V), V}$ under the mapping $\tilde{\eta}$.

Assumption 2c. (Smooth effect of $V) y_{2}(t, v, u)$ is continuous on $I_{V, U}$ for each $t=0,1$.

Assumption 3c. (First stage and non-negligible population at the kink) (i) $b(\cdot)$ is a known function, everywhere continuous and continuously differentiable on $I_{V} \backslash\{0\}$, but $\lim _{v \rightarrow 0^{+}} b^{\prime}(v) \neq \lim _{v \rightarrow 0^{-}} b^{\prime}(v)$. (ii) The set $A_{U} \equiv\left\{u: f_{V, \eta \mid U=u}(v, n)>0 \forall(v, n) \in I_{V, \tilde{\eta}(b(V), V)}\right\}$ has a positive measure under $U: \int_{A_{U}} d F_{U}(u)>0$. (iii) $t_{1}\left(b_{0}, 0, n^{0}\right) \neq 0$.

Assumption 4c. (Smooth density) The conditional density $f_{V, \eta \mid U=u}(v, n)$ and its partial derivative w.r.t. $v, \frac{\partial f_{V, \eta \mid U=u}(v, n)}{\partial v}$, are continuous on $I_{V, \eta, U}$.

Proposition 3. Under Assumptions 1c-4c:

(a) $\operatorname{Pr}(U \leqslant u \mid V=v)$ is continuously differentiable in $v$ at $v=0 \forall u \in I_{U}$.

(b)

$$
\frac{\left.\lim _{v_{0} \rightarrow 0^{+}} \frac{d E[Y \mid V=v]}{d v}\right|_{v=v_{0}}-\left.\lim _{v_{0} \rightarrow 0^{-}} \frac{d E[Y \mid V=v]}{d v}\right|_{v=v_{0}}}{\left.\lim _{v_{0} \rightarrow 0^{+}} \frac{d E[T \mid V=v]}{d v}\right|_{v=v_{0}}-\left.\lim _{v_{0} \rightarrow 0^{-}} \frac{d E[T \mid V=v]}{d v}\right|_{v=v_{0}}}=\int\left[y(1,0, u)-y(0,0, u) \frac{f_{V, \eta \mid U=u}\left(0, n^{0}\right)}{f_{V, \eta}\left(0, n^{0}\right)} d F_{U}(u) .\right.
$$

Proof: The proof of (a) is analogous to that of Proposition 1(a).

For part (b), note that

$$
\begin{aligned}
\frac{d}{d v} E[T \mid V=v]= & \frac{d}{d v} E\left[1_{\left[T^{*} \geqslant 0\right]} \mid V=v\right] \\
= & \frac{d}{d v} \int_{\tilde{\eta}(b(v), v)}^{d_{\eta}} f_{\eta \mid V=v}(n) d n \\
= & \int_{\tilde{\eta}(b(v), v)}^{d_{\eta}} \frac{\partial}{\partial v}\left[f_{\eta \mid V=v}(n)\right] d n \\
& -\left[\tilde{\eta}_{1}(b(v), v) b^{\prime}(v)+\tilde{\eta}_{2}(b(v), v)\right] f_{\eta \mid V=v}(\tilde{\eta}(b(v), v)),
\end{aligned}
$$

where $\tilde{\eta}_{k}$ denotes the the partial derivative of $\tilde{\eta}$ with respect to its $k$-th argument. The second line follows from Assumption 1c, and the interchange of differentiation and integration in the third line is permitted by 
Assumption 4c. It follows that the denominator can be expressed as

$$
\begin{aligned}
\left.\lim _{v_{0} \rightarrow 0^{+}} \frac{d E[T \mid V=v]}{d v}\right|_{v=v_{0}}-\left.\lim _{v_{0} \rightarrow 0^{-}} \frac{d E[T \mid V=v]}{d v}\right|_{v=v_{0}} & =-\left[\lim _{v_{0} \rightarrow 0^{+}} b^{\prime}\left(v_{0}\right)-\lim _{v_{0} \rightarrow 0^{-}} b^{\prime}\left(v_{0}\right)\right] \tilde{\eta}_{1}\left(b_{0}, 0\right) f_{\eta \mid V=0}\left(\tilde{\eta}\left(b_{0}, 0\right)\right) \\
& =-\left[\lim _{v_{0} \rightarrow 0^{+}} b^{\prime}\left(v_{0}\right)-\lim _{v_{0} \rightarrow 0^{-}} b^{\prime}\left(v_{0}\right)\right] \tilde{\eta}_{1}\left(b_{0}, 0\right) \frac{f_{V, \eta}\left(0, n^{0}\right)}{f_{V}(0)} .
\end{aligned}
$$

Similarly, by Assumptions 1c, 2c and 4c,

$$
\begin{aligned}
\frac{d}{d v} E[Y \mid V=v]= & \frac{d}{d v} E[y(T, V, U) \mid V=v] \\
= & \frac{d}{d v} \int\left\{\int_{\tilde{\eta}(b(v), v)}^{d_{\eta}} y(1, v, u) f_{\eta \mid V=v, U=u}(n) d n\right. \\
& \left.+\int_{c_{\eta}}^{\tilde{\eta}(b(v), v)} y(0, v, u) f_{\eta \mid V=v, U=u}(n) d n\right\} d F_{U \mid V=v}(u) \\
= & \int\left\{\int_{\tilde{\eta}(b(v), v)}^{d_{\eta}} \frac{\partial}{\partial v}\left[y(1, v, u) f_{\eta \mid V=v, U=u}(n)\right] d n\right. \\
& \left.+\int_{c_{\eta}}^{\tilde{\eta}(b(v), v)} \frac{\partial}{\partial v}\left[y(0, v, u) f_{\eta \mid V=v, U=u}(n)\right] d n\right\} d F_{U \mid V=v}(u) \\
& -\int[y(1, v, u)-y(0, v, u)] f_{\eta \mid V=v, U=u}(\tilde{\eta}(b(v), v))\left[\tilde{\eta}_{1}(b(v), v) b^{\prime}(v)+\tilde{\eta}_{2}(b(v), v)\right] d F_{U \mid V=v}(u),
\end{aligned}
$$

and it follows that the numerator is

$$
\begin{aligned}
& \left.\lim _{v_{0} \rightarrow 0^{+}} \frac{d E[Y \mid V=v]}{d v}\right|_{v=v_{0}}-\left.\lim _{v_{0} \rightarrow 0^{-}} \frac{d E[Y \mid V=v]}{d v}\right|_{v=v_{0}} \\
=\quad & -\left[\lim _{v_{0} \rightarrow 0^{+}} b^{\prime}\left(v_{0}\right)-\lim _{v_{0} \rightarrow 0^{-}} b^{\prime}\left(v_{0}\right)\right] \tilde{\eta}_{1}\left(b_{0}, 0\right) \\
& \int[y(1,0, u)-y(0,0, u)] f_{\eta \mid V=0, U=u}\left(n^{0}\right) d F_{U \mid V=0}(u) \\
= & -\left[\lim _{v_{0} \rightarrow 0^{+}} b^{\prime}\left(v_{0}\right)-\lim _{v_{0} \rightarrow 0^{-}} b^{\prime}\left(v_{0}\right)\right] \tilde{\eta}_{1}\left(b_{0}, 0\right) \\
& \int[y(1,0, u)-y(0,0, u)] \frac{f_{V, \eta \mid U=u}\left(0, n^{0}\right)}{f_{V}(0)} d F_{U}(u) .
\end{aligned}
$$

Assumption $3 \mathrm{c}$ (iii) and the implicit function theorem imply that $\tilde{\eta}_{1}\left(b_{0}, 0\right) \neq 0$, and therefore,

$$
\frac{\left.\lim _{v_{0} \rightarrow 0^{+}} \frac{d E[Y \mid V=v]}{d v}\right|_{v=v_{0}}-\left.\lim _{v_{0} \rightarrow 0^{-}} \frac{d E[Y \mid V=v]}{d v}\right|_{v=v_{0}}}{\left.\lim _{v_{0} \rightarrow 0^{+}} \frac{d E[T \mid V=v]}{d v}\right|_{v=v_{0}}-\left.\lim _{v_{0} \rightarrow 0^{-}} \frac{d E[T \mid V=v]}{d v}\right|_{v=v_{0}}}=\int[y(1,0, u)-y(0,0, u)] \frac{f_{V, \eta \mid U=u}\left(0, n^{0}\right)}{f_{V, \eta}\left(0, n^{0}\right)} d F_{U}(u)
$$

by Assumption 3c.

When the benefit variable $b$ directly affects the outcome, i.e. $Y=y(T, B, V, U)$, the fuzzy RKD estimand no longer identifies the causal effect of $T$ on $Y$; rather, the effect of $T$ on $Y$ is confounded by the direct effect of $B$ on $Y$. If Assumptions 1c-4c are modified accordingly, it can be shown that the RK estimand identifies the parameter 


$$
\underbrace{\int[y(1,0, u)-y(0,0, u)] \frac{f_{V, \eta \mid U=u}\left(0, n^{0}\right)}{f_{V, \eta}\left(0, n^{0}\right)} d F_{U}(u)}_{(i)}-\underbrace{\frac{E\left[y_{2}(T, b(V), V, U) \mid V=0\right]}{\tilde{\eta}_{1}\left(b_{0}, 0\right) f_{\eta \mid V=0}\left(n_{0}\right)}}_{(i i)},
$$

where term (i) is the same as the RHS of equation (16) and term (ii) is the component that depends on the direct impact of $B$ on $Y$. To the extent that the researcher can determine the sign of (ii), which involves signing $E\left[y_{2}(T, b(V), V, U) \mid V=0\right]$ and $\tilde{\eta}_{1}\left(b_{0}, 0\right)$, she can bound the treatment effect (i) with the RKD estimand. For example, when $\eta$ represents a student's ability in the empirical example in Remark 6, we may assert that $\tilde{\eta}_{1}\left(b_{0}, 0\right)<0$ because the expected return from college attendance increases with the amount of financial aid. The conditional expectation of the direct impact of $B$ on $Y, E\left[y_{2}(T, b(V), V, U) \mid V=0\right]$, may be positive because a more generous aid package allows a student more time to focus on her study. If these arguments were true, then the RKD estimand would serve as an upper bound on the economic returns to college attendance.

As stated in Remark 6, we can also allow the relationship between $B$ and $V$ to be fuzzy as in section 2.2.2.: $B=b(V, \varepsilon)$. In addition, we allow measurement error in $V, \underline{U_{V}}$, which has a point mass at 0 , and we only observe $V^{*}=V+U_{V}$. We do not need to consider the measurement error in $B$ since the observed value of $B$ does not appear in the RK estimand. We abstract away from potential measurement error in $T$ and leave it for future research. The modified set of identifying assumptions are:

Assumption 1d. (Regularity) In addition to the conditions in Assumption 1c, the support of $\varepsilon$ is bounded: it is a subset of the arbitrarily large compact set $I_{\mathcal{E}} \subset \mathbb{R}^{k}$.

Assumption 3d. (First stage and non-negligible population at the kink) (i) $b(v, e)$ is continuous on $I_{V, \varepsilon}$ and $b_{1}(v, e)$ is continuous on $\left(I_{V} \backslash\{0\}\right) \times I_{\varepsilon}$. Let $b_{1}^{+}(e) \equiv \lim _{v \rightarrow 0^{+}} b_{1}(v, e), b_{1}^{-}(e) \equiv \lim _{v \rightarrow 0^{-}} b_{1}(v, e), A_{\varepsilon} \equiv\{e$ : $\left.f_{V \mid \varepsilon=e}(0)>0\right\}$ and $n^{0}(e) \equiv \tilde{\eta}(b(0, e), 0)$, then $\int\left\{\left|b_{1}^{+}(e)-b_{1}^{-}(e)\right|\left|\tilde{\eta}_{1}(b(0, e), 0)\right| \operatorname{Pr}\left[U_{V}=0 \mid V=0, \varepsilon=e, \eta=\right.\right.$ $\left.n^{0}(e)\right] f_{V, \eta \mid \varepsilon=e}\left(0, n^{0}(e)\right) d F_{\varepsilon}(e)>0$.

Assumption 4d. (Smooth density) The conditional density $f_{V, \eta, U_{V^{\prime}} \mid U=u, \varepsilon=e}\left(v, n, u_{V^{\prime}}\right)$ and its partial derivative w.r.t. $v, \frac{\partial f_{V, \eta, U_{V^{\prime}} \mid U=u, \varepsilon=e}\left(v, n, u_{V^{\prime}}\right)}{\partial v}$, are continuous on $I_{V, \eta, U_{V^{\prime}}, U, \varepsilon}$.

Assumption 5d. (Smooth probability of no error in $V$ and $B$ ) As a function of the realized values of $V, U, \varepsilon, \eta$ and $U_{V^{\prime}}$, the conditional probability of $U_{V}=0$, denoted by $\pi\left(v, u, e, u_{V^{\prime}}, u_{B^{\prime}}\right)$, and its partial derivative w.r.t. $v$ are continuous on $I_{V, U, \varepsilon, \eta, U_{V^{\prime}}}$.

Assumption 6d. (Monotonicity) (i) Either $b_{1}^{+}(e) \geq b_{1}^{-}(e)$ for all $e$ or $b_{1}^{+}(e) \leq b_{1}^{-}(e)$ for all $e$. (ii) $t_{1}\left(b(0, e), 0, n^{0}(e)\right) \geqslant 0$ for all $e$ or $t_{1}\left(b(0, e), 0, n^{0}(e)\right) \leqslant 0$ for all $e$. 
Proposition 4. Under Assumptions 1d, 2, 3d-6d:

(a) $\operatorname{Pr}\left(U \leqslant u, \varepsilon=e, \eta=n \mid V^{*}=v^{*}\right)$ is continuously differentiable in $v^{*}$ at $v^{*}=0 \forall(u, e, n) \in I_{U, \varepsilon, \eta}$.

(b)

$$
\frac{\left.\lim _{v_{0} \rightarrow 0^{+}} \frac{d E[Y \mid V=v]}{d v}\right|_{v=v_{0}}-\left.\lim _{v_{0} \rightarrow 0^{-}} \frac{d E[Y \mid V=v]}{d v}\right|_{v=v_{0}}}{\left.\lim _{v_{0} \rightarrow 0^{+}} \frac{d E[T \mid V=v]}{d v}\right|_{v=v_{0}}-\left.\lim _{v_{0} \rightarrow 0^{-}} \frac{d E[T \mid V=v]}{d v}\right|_{v=v_{0}}}=\int[y(1,0, u)-y(0,0, u)] \tilde{\varphi}(u, e) d F_{U, \varepsilon}(u, e),
$$

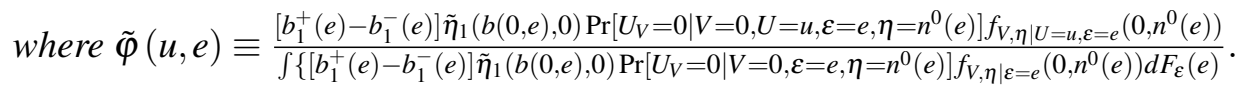

Proof: The proof is similar to that of Proposition 2 and is omitted.

\section{B Estimation}

\section{B.1 Two-sample RKD}

As suggested by a referee, the triplet $(Y, B, V)$ may not be jointly observed from a single data source. Instead, the vectors $\left(Y_{i}, V_{i}\right)$ for $i=1, \ldots, n_{1}$ are observed in data set 1 and $\left(B_{j}, V_{j}\right)$ for $j=1, \ldots, n_{2}$ are observed in data set 2. Because of the requirement of a zero point mass in the $U_{V}$ distribution in Assumption 3a, an RKD typically calls for administrative data as opposed to surveys based on a complex sampling design. Therefore, we assume that $\left(Y_{i}, V_{i}\right)$ and $\left(B_{j}, V_{j}\right)$ are independent i.i.d. samples as per Inoue and Solon (2010). The variances of the first-stage and reduced-form kink estimators, $\hat{\tau}_{B}=\hat{\kappa}_{1}^{+}-\hat{\kappa}_{1}^{-}$and $\hat{\tau}_{Y}=\hat{\beta}_{1}^{+}-\hat{\beta}_{1}^{-}$can be calculated by using the sharp RKD variance estimator, and the covariance between $\hat{\tau}_{B}$ and $\hat{\tau}_{Y}$ is zero by the

independence assumption. It follows that the variance of the fuzzy RKD estimator $\frac{\hat{\tau}_{Y}}{\hat{\tau}_{B}}$ can be calculated by an application of the delta method. The robust confidence intervals in Calonico et al. (Forthcoming) can be constructed analogously by setting the covariances between the first-stage and reduced-form estimators to zero.

\section{B.2 Optimal Bandwidth in Fuzzy RKD}

In this section, we propose bandwidth selectors that minimize the asymptotic MSE of the fuzzy RD/RKD estimators, building on that in Imbens and Kalyanaraman (2012) (henceforth IK bandwidth) and Calonico et al. (Forthcoming) (henceforth CCT bandwidth). First we introduce notation similar to Calonico et al. (Forthcoming). Define $\mu_{-+}^{(v)}$ and $\mu_{--}^{(v)}$ as the $v$-th right and left derivatives of the conditional expectation of a random variable $(Y$ or $B)$ with respect to $V$ at $V=0$; let $\tau_{Y, v} \equiv \mu_{Y+}^{(v)}-\mu_{Y-}^{(v)}$ and $\tau_{B, v} \equiv \mu_{B+}^{(v)}-\mu_{B-}^{(v)}$. In addition, let $\sigma_{Y+}^{2}, \sigma_{Y-}^{2}, \sigma_{B+}^{2}, \sigma_{B-}^{2}, \sigma_{Y B+}$ and $\sigma_{Y B-}$ be the conditional variances of $Y$ and $B$ and their 
conditional covariance on two sides of the threshold. Finally, let

$$
\begin{aligned}
\tilde{\zeta}_{v, p, s}(h)= & \frac{1}{\tau_{B, v}}\left[\left(\hat{\mu}_{Y+}^{(v)}(h)-(-1)^{s} \hat{\mu}_{Y-}^{(v)}(h)\right)-\left(\mu_{Y+}^{(v)}-(-1)^{s} \mu_{Y-}^{(v)}\right)\right] \\
& +\frac{\tau_{Y, v}}{\tau_{B, v}^{2}}\left[\left(\hat{\mu}_{B+}^{(v)}(h)-(-1)^{s} \hat{\mu}_{B-}^{(v)}(h)\right)-\left(\mu_{B+}^{(v)}-(-1)^{s} \mu_{B-}^{(v)}\right)\right],
\end{aligned}
$$

where $\hat{\mu}_{\cdot+}^{(v)}$ and $\hat{\mu}_{.-}^{(v)}$ are the $p$-th order local polynomial estimator of $\mu_{++}^{(v)}$ and $\mu_{--}^{(v)}$ respectively.

Next we propose the lemma that generalizes Lemma 2 of Calonico et al. (Forthcoming) and serves as the fuzzy analog of its Lemma 1:

Lemma 7. Assume that Assumptions 1-3 in Calonico et al. (Forthcoming) are satisfied with $S \geqslant p+1$ and $v \leqslant p$. If $h \rightarrow 0$ and $n h \rightarrow \infty$, then $M S E_{v, p, s}=E\left[\left(\tilde{\varsigma}_{v, p, s}(h)\right)^{2} \mid\left\{V_{i}\right\}_{i=1}^{n}\right]=h^{2(p+1-v)}\left[B_{F, v, p, p+1, s}^{2}+o_{p}(1)\right]+$ $\frac{1}{n h_{n}^{1+2 v}}\left[V_{F, v, p}+o_{p}(1)\right]$ where

$$
\begin{gathered}
B_{F, v, p, r, s}=\left(\frac{1}{\tau_{B, v}} \frac{\mu_{Y+}^{(r)}-(-1)^{v+r+s} \mu_{Y-}^{(r)}}{r !}-\frac{\tau_{Y, v}}{\tau_{B, v}^{2}} \frac{\mu_{B+}^{(r)}-(-1)^{v+r+s} \mu_{B-}^{(r)}}{r !}\right) ! e_{v}^{\prime} \Gamma_{p}^{-1} \vartheta_{p, r} \\
V_{F, v, p}=\left(\frac{1}{\tau_{B, v}^{2}} \frac{\sigma_{Y-}^{2}+\sigma_{Y+}^{2}}{f}-\frac{2 \tau_{Y, v}}{\tau_{B, v}^{3}} \frac{\sigma_{Y B-}+\sigma_{Y B+}}{f}+\frac{\tau_{Y, v}^{2}}{\tau_{B, v}^{4}} \frac{\sigma_{B-}^{2}+\sigma_{B+}^{2}}{f}\right) v !^{2} e_{v}^{\prime} \Gamma_{p}^{-1} \Psi_{p} \Gamma_{p}^{-1} e_{v}
\end{gathered}
$$

with $e_{v}, \Gamma_{p}, \Psi_{p}$ and $\vartheta_{p, r}$ as defined in Calonico et al. (Forthcoming). If, in addition, $\mathrm{B}_{F, v, p, r, s} \neq 0$, then the asymptotic MSE-optimal bandwidth is $h_{M S E, F, v, p}=C_{F, v, p, s}^{\frac{1}{2 p+3}} n^{-\frac{1}{2 p+3}}$ where $C_{F, v, p, s}=\frac{(2 v+1) V_{F, v, p}}{2(p+1-v) B_{F, v, p, p+1, s}^{2}}$.

Proof. The proof of Lemma 7 is analogous to that of Lemma A2 of Calonico et al. (Forthcoming).

Note that Lemma 2 of Calonico et al. (Forthcoming) is a special case of Lemma 7 above with $s=0$. As in the sharp case, the bias of the fuzzy RD estimator depends on the difference or sum of the derivative estimator from the first stage and the outcome equation. Whether it is a difference or sum depends the order of the derivative estimated as well as the order of the estimating polynomial. Based on Lemma 7, we propose procedures to compute the CCT and IK bandwidths adapted to the fuzzy RD/RKD designs in the two following subsections. 


\section{B.2.1 Fuzzy bandwidth based on the CCT procedure}

Define the local variance estimator

$$
\hat{\mathrm{V}}_{F, v, p}(h)=\frac{1}{\tilde{\tau}_{B, v}^{2}} \hat{\mathrm{V}}_{Y Y, v, p}(h)-\frac{2 \tilde{\tau}_{Y, v}}{\tilde{\tau}_{B, v}^{3}} \hat{\mathrm{V}}_{Y B, v, p}(h)+\frac{\tilde{\tau}_{Y, v}^{2}}{\tilde{\tau}_{B, v}^{4}} \hat{\mathrm{V}}_{B B, v, p}(h),
$$

where

$$
\begin{aligned}
\hat{V}_{R_{1} R_{2}, v, p}(h)= & \hat{\mathscr{V}}_{R_{1} R_{2}+, v, p}(h)+\hat{\mathscr{V}}_{R_{1} R_{2}-, v, p}(h) \\
= & v !^{2} e_{v}^{\prime} \Gamma_{+, p}^{-1}(h) \hat{\Psi}_{R_{1} R_{2}+, p}(h) \Gamma_{+, p}^{-1}(h) e_{v} / n h^{2 v} \\
& +v !^{2} e_{v}^{\prime} \Gamma_{-, p}^{-1}(h) \hat{\Psi}_{R_{1} R_{2}-, p}(h) \Gamma_{-, p}^{-1}(h) e_{v} / n h^{2 v},
\end{aligned}
$$

with $R_{1}$ and $R_{2}$ serving as place holders for $Y$ and $B$, and the quantities $e_{\nu}, \Gamma_{+, p}(h)$ and $\hat{\Psi}_{R_{1} R_{2}+, p}(h)$ as defined in Calonico et al. (Forthcoming). The constants $\Gamma_{p}, \vartheta_{p, q}, \mathscr{B}_{v, p}$ and $C_{v, p}(K)$ also follow the same definitions in Calonico et al. (Forthcoming).

Step 0: Use the CCT bandwidth (optimal in the MSE sense for estimating $\tau_{Y, v}$ ) to obtain preliminary estimates $\tilde{\tau}_{Y, v}$ and $\tilde{\tau}_{B, v}$.

Step 1: $v$ and $c$

1. $v=$ Const $_{K} \cdot \min \left\{S_{V}, I Q R_{V} / 1.349\right\} \cdot n^{-1 / 5}$ where Const $_{K}=\left(\frac{8 \sqrt{\pi} \int K(u)^{2} d u}{3\left(\int u^{2} K(u) d u\right)^{2}}\right)^{1 / 5} ; S_{V}^{2}$ and $I Q R_{V}$ denote the sample variance and interquartile range of $V$. The selection of $v_{n}$, which is based on the Silverman's rule of thumb, is the same as in Calonico et al. (Forthcoming). Use $v$ to compute the variance estimator $\hat{\mathrm{V}}_{F, q+1, q+1}(\hat{v}), \hat{\mathrm{V}}_{F, p+1, q}(\hat{v})$ and $\hat{\mathrm{V}}_{F, v, p}(\hat{v})$.

2. Run global polynomials of order $q+2$ separately for $B$ and $Y$ on each side of the threshold. Obtain estimators of the $(q+2)$-th derivatives on both sides of the threshold $e_{q+2}^{\prime} \hat{\gamma}_{Y \pm, q+2}$ and $e_{q+2}^{\prime} \hat{\gamma}_{B \pm, q+2}$,

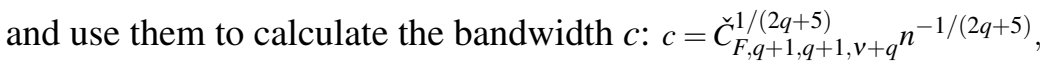

$\check{C}_{F, q+1, q+1, v+q}=\frac{(2 q+3) n v_{n}^{2 q+3} \hat{V}_{F, q+1, q+1}(\hat{v})}{2 \mathscr{B}_{q+1, q+1}^{2}\left\{\frac{1}{\bar{t}_{B, v}}\left[e_{q+2}^{\prime} \hat{\gamma}_{Y+, q+2}-(-1)^{v+q} e_{q+2}^{\prime} \hat{\gamma}_{Y-, q+2}\right]-\frac{\bar{T}_{Y, v}}{\tilde{\tau}_{B, v}^{2}}\left[e_{q+2}^{\prime} \hat{\gamma}_{B+, q+2}-(-1)^{v+q} e_{q+2}^{\prime} \hat{\gamma}_{B-, q+2}\right]\right\}^{2}}$.

Step 2: $h_{q}$

Perform local regressions with bandwidth $c$ to estimate the $(q+1)$-th derivatives on both sides of the threshold and calculate bandwidth $h_{q}: \hat{h}_{q}=\hat{C}_{F, p+1, q, v+q+1}^{1 /(2 q+3)} n^{-1 /(2 q+3)}$,

$\hat{C}_{F, p+1, q, v+q+1}=\frac{(2 p+3) n v_{n}^{2 p+3} \hat{V}_{F, p+1, q}(\hat{v})}{2(q-p) \mathscr{B}_{p+1, q}^{2}\left\{\frac{1}{\tau_{B, v}}\left[e_{q+1}^{\prime} \hat{\beta}_{Y+, q+1}(\hat{c})-(-1)^{v+q+1} e_{q+1}^{\prime} \hat{\beta}_{Y-, q+1}(\hat{c})\right]-\frac{\bar{T}_{,, v}}{\tilde{\tau}_{B, v}^{2}}\left[e_{q+1}^{\prime} \hat{\beta}_{B+, q+1}(\hat{c})-(-1)^{v+q+1} e_{q+1}^{\prime} \hat{\beta}_{B-, q+1}(\hat{c})\right]\right\}^{2}}$.

Step 3: $h$ 
Perform local regression with bandwidth $h_{q}$ to estimate the bias in the fuzzy RD/RKD estimator $\hat{\tau}_{F, v, p}$ and calculate the resulting main bandwidth $h: \hat{h}=\hat{C}_{F, v, p, v+p+1}^{1 /(2 p+3)} n^{-1 /(2 p+3)}$,

$\hat{C}_{F, v, p, v+p+1}=\frac{(2 v+1) n v_{n}^{2 v+1} \hat{V}_{F, v, p}(\hat{v})}{2(p+1-v) \mathscr{B} \hat{F}_{v, p}^{2}\left\{\frac{1}{\tau_{B_{, v}, v}}\left[e_{p+1}^{\prime} \hat{\beta}_{Y+, q}\left(\hat{h}_{q}\right)-(-1)^{v+p+1} e_{p+1}^{\prime} \hat{\beta}_{Y-q}\left(\hat{h}_{q}\right)\right]-\frac{t_{Y, v}}{\tau_{B_{B, v}}^{2}}\left[e_{p+1}^{\prime} \hat{\beta}_{B+, q}\left(\hat{h}_{q}\right)-(-1)^{v+p+1} e_{p+1}^{\prime} \hat{\beta}_{B-q, q}\left(\hat{h}_{q}\right)\right]\right]^{2}}$.

Similar to Calonico et al. (Forthcoming), we have the following consistency result for the fuzzy CCT bandwidth selectors proposed above.

Proposition 5. (Consistency of the CCT Bandwidth Selectors) Let $v \leqslant p<q$. Suppose Assumptions 1-3 in Calonico et al. (Forthcoming) hold with $S \geqslant q+2$ and that

$$
\frac{1}{\tilde{\tau}_{B, v}}\left[e_{q+2}^{\prime} \hat{\gamma}_{Y+, q+2}-(-1)^{v+q} e_{q+2}^{\prime} \hat{\gamma}_{Y-, q+2}\right]-\frac{\tilde{\tau}_{Y, v}}{\tilde{\tau}_{B, v}^{2}}\left[e_{q+2}^{\prime} \hat{\gamma}_{B+, q+2}-(-1)^{v+q} e_{q+2}^{\prime} \hat{\gamma}_{B-, q+2}\right] \stackrel{p}{\rightarrow} c \neq 0 .
$$

Step 1. If $\mathrm{B}_{F, p+1, q, q+1, v+p+1} \neq 0$, then

$$
\frac{\hat{h}_{q}}{h_{\mathrm{MSE}, F, p+1, q, v+p+1}} \stackrel{p}{\rightarrow} 1 \text { and } \frac{\operatorname{MSE}_{F, p+1, q, v+p+1}\left(\hat{h}_{q}\right)}{\operatorname{MSE}_{p+1, q, v+p+1}\left(h_{\mathrm{MSE}, p+1, q, v+p+1}\right)} \stackrel{p}{\rightarrow} 1
$$

Step 2. If $\mathrm{B}_{F, v, p, p+1,0} \neq 0$, then

$$
\frac{\hat{h}}{h_{\mathrm{MSE}, F, v, p, 0}} \stackrel{p}{\rightarrow} 1 \text { and } \frac{\operatorname{MSE}_{F, v, p, 0}(\hat{h})}{\operatorname{MSE}_{F, v, p, 0}\left(h_{\mathrm{MSE}, F, v, p, 0}\right)} \stackrel{p}{\rightarrow} 1
$$

Proof: Because the CCT bandwidth optimal for estimating $\tau_{Y, v}$ shrinks at the rate of $n^{-\frac{1}{2 p+3}}$, the preliminary estimators, $\tilde{\tau}_{Y, v}$ and $\tilde{\tau}_{B, v}$, are consistent. The rest of the proof follows the arguments in the proof of Theorem A4 in Calonico et al. (Forthcoming).

The optimal fuzzy RD bandwidth is proposed in Imbens and Kalyanaraman (2012). We suggest an extension to be used in the fuzzy RKD case $(v=1)$ and state the bandwidth selectors for a generic $v$. Calonico et al. (Forthcoming), Calonico et al. (in press) and Calonico et al. (2014b) adapt the IK bandwidth selection procedure to $h_{q}$ so that it can be used to bias-correct the RD estimator. Building upon these studies, we propose a further extension of the bandwidth selector for $h_{q}$ to a general fuzzy design with a discontinuity in the $v$-th derivative. 


\section{B.2.2 Fuzzy bandwidth based on the IK procedure}

Step 1: Use the sharp IK bandwidth (optimal in the MSE sense for estimating $\tau_{Y, v}$ ) to obtain preliminary estimates $\tilde{\tau}_{Y, v}$ and $\tilde{\tau}_{B, v}$.

Step 2: $v$

1. $\hat{v}=1.84 \cdot S_{V} \cdot n^{-1 / 5}$

2. Use $\hat{h}_{1}$ to estimate $\hat{\sigma}_{Y \pm}^{2}(\hat{v}), \hat{\sigma}_{B \pm}^{2}(\hat{v}), \hat{\sigma}_{Y B \pm}(\hat{v})$ and $\hat{f}(\hat{v})$ as specified in Imbens and Kalyanaraman (2012) (note that Imbens and Kalyanaraman (2012) use $W$ to denote the treatment variable and use $h_{1}$ to denote this preliminary bandwidth).

Step 3: $h_{q}$

Run global regressions:

$$
\begin{aligned}
& Y=\delta^{Y} \cdot 1_{[V \geqslant 0]} \cdot V^{V}+\alpha_{0}^{Y}+\alpha_{1}^{Y} V+\ldots+\alpha_{q+2}^{Y} V^{q+2}+\varepsilon^{Y} \\
& B=\delta^{B} \cdot 1_{[V \geqslant 0]} \cdot V^{V}+\alpha_{0}^{B}+\alpha_{1}^{B} V+\ldots+\alpha_{q+2}^{B} V^{q+2}+\varepsilon^{B}
\end{aligned}
$$

and use $\hat{\alpha}_{q+2}^{Y}$ and $\hat{\alpha}_{q+2}^{B}$ to construct

- $\hat{h}_{Y-, q+1}=\left(C_{q+1, q+1}\left(K_{U}\right) \frac{\hat{\sigma}_{Y-}^{2}(\hat{v}) / \hat{f}(\hat{v})}{n_{-}\left(\hat{\alpha}_{q+2}^{Y}\right)^{2}}\right)^{\frac{1}{2 q+5}}$

- $\hat{h}_{Y+, q+1}=\left(C_{q+1, q+1}\left(K_{U}\right) \frac{\hat{\sigma}_{Y+}^{2}(\hat{v}) / \hat{f}(\hat{v})}{n_{+}\left(\hat{\alpha}_{q+2}^{Y}\right)^{2}}\right)^{\frac{1}{2 q+5}}$

- $\hat{h}_{B-, q+1}=\left(C_{q+1, q+1}\left(K_{U}\right) \frac{\hat{\sigma}_{B-}^{2}(\hat{v}) / \hat{f}(\hat{v})}{n_{-}\left(\hat{\alpha}_{q+2}^{B}\right)^{2}}\right)^{\frac{1}{2 q+5}}$

- $\hat{h}_{B+, q+1}=\left(C_{q+1, q+1}\left(K_{U}\right) \frac{\hat{\sigma}_{B+}^{2}(\hat{v}) / \hat{f}(\hat{v})}{n_{+}\left(\hat{\alpha}_{q+2}^{B}\right)^{2}}\right)^{\frac{1}{2 q+5}}$.

Perform $(q+1)$-th order local regressions of $Y$ and $B$ on each side of the threshold with the uniform kernel $K_{U}$ and bandwidths $\hat{h}_{Y \pm, q+1}$ and $\hat{h}_{B \pm, q+1}$. Using in the resulting estimators $\hat{\beta}_{Y \pm, q+1}\left(\hat{h}_{Y \pm, q+1}\right)$ and $\hat{\beta}_{B \pm, q+1}\left(\hat{h}_{B \pm, q+1}\right)$, we obtain $\hat{h}_{q}=\hat{C}_{F, p+1, q, v+q+1}^{1 /(2 q+3)} n^{-1 /(2 q+3)}$,

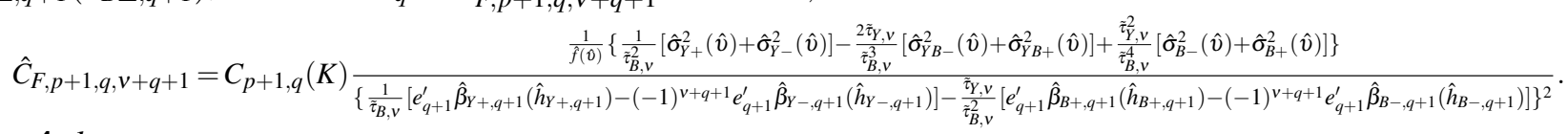

Step 4: $h$ 
Run global regressions:

$$
\begin{aligned}
Y & =\delta^{Y} \cdot 1_{[V \geqslant 0]} \cdot V^{v}+\gamma_{0}^{Y}+\gamma_{1}^{Y} V+\ldots+\gamma_{q+1}^{Y} V^{q+1}+\varepsilon^{Y} \\
B & =\delta^{B} \cdot 1_{[V \geqslant 0]} \cdot V^{v}+\gamma_{0}^{B}+\gamma_{1}^{B} V+\ldots+\gamma_{q+1}^{B} V^{q+1}+\varepsilon^{B}
\end{aligned}
$$

and use $\hat{\gamma}_{q+1}^{Y}$ and $\hat{\gamma}_{q+1}^{B}$ to construct

- $\hat{h}_{Y-, q}=\left(C_{p+1, q}\left(K_{U}\right) \frac{\hat{\sigma}_{Y-}^{2}(\hat{v}) / \hat{f}(\hat{v})}{n_{-}\left(\hat{\gamma}_{q+1}^{Y}\right)^{2}}\right)^{\frac{1}{2 q+3}}$

- $\hat{h}_{Y+, q}=\left(C_{p+1, q}\left(K_{U}\right) \frac{\hat{\sigma}_{Y+}^{2}(\hat{v}) / \hat{f}(\hat{v})}{n_{+}\left(\hat{\gamma}_{q+1}^{Y}\right)^{2}}\right)^{\frac{1}{2 q+3}}$

- $\hat{h}_{B-, q}=\left(C_{p+1, q}\left(K_{U}\right) \frac{\hat{\sigma}_{B-}^{2}(\hat{v}) / \hat{f}(\hat{v})}{n_{-}\left(\hat{\gamma}_{q+1}^{B}\right)^{2}}\right)^{\frac{1}{2 q+3}}$

- $\hat{h}_{B+, q}=\left(C_{p+1, q}\left(K_{U}\right) \frac{\hat{\sigma}_{B+}^{2}(\hat{v}) / \hat{f}(\hat{v})}{n_{+}\left(\hat{\gamma}_{q+1}^{B}\right)^{2}}\right)^{\frac{1}{2 q+3}}$.

Perform $q$-th order local regressions of $Y$ and $B$ on each side of the threshold with bandwidths $\hat{h}_{Y \pm, q}$ and $\hat{h}_{B \pm, q}$ and obtain local regression estimators $\hat{\beta}_{Y \pm, q}\left(\hat{h}_{Y \pm, q}\right)$ and $\hat{\beta}_{B \pm, q}\left(\hat{h}_{B \pm, q}\right)$. Plugging them in, we have an estimate of the main bandwidth $h: \hat{h}=\hat{C}_{F, v, p, v+p+1}^{1 /(2 p+3)} n^{-1 /(2 p+3)}$,

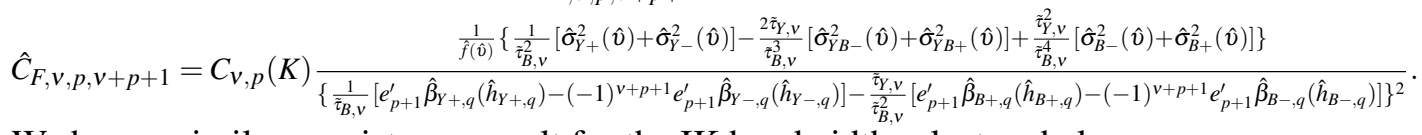

We have a similar consistency result for the IK bandwidth selectors below.

Proposition 6. (Consistency of the IK Bandwidth Selectors) Let $v \leqslant p<q$. Suppose Assumptions 1-3 in Calonico et al. (Forthcoming) hold with $S \geqslant q+2$ and that $\alpha_{q+2}^{Y}, \alpha_{q+2}^{B}, \gamma_{q+1}^{Y}$ and $\gamma_{q+1}^{B}$ are nonzero.

Selector for $h_{q}$ : If $\mathrm{B}_{F, p+1, q, q+1, v+p+1} \neq 0$, then

$$
\frac{\hat{h}_{q}}{h_{\mathrm{MSE}, F, p+1, q, v+p+1}} \stackrel{p}{\rightarrow} 1 \text { and } \frac{\operatorname{MSE}_{F, p+1, q, v+p+1}\left(\hat{h}_{q}\right)}{\operatorname{MSE}_{p+1, q, v+p+1}\left(h_{\mathrm{MSE}, p+1, q, v+p+1}\right)} \stackrel{p}{\rightarrow} 1
$$

Selector for $h$. If $\mathrm{B}_{F, v, p, p+1,0} \neq 0$, then

$$
\frac{\hat{h}}{h_{\mathrm{MSE}, F, v, p, 0}} \stackrel{p}{\rightarrow} 1 \text { and } \frac{\operatorname{MSE}_{F, v, p, 0}(\hat{h})}{\operatorname{MSE}_{F, v, p, 0}\left(h_{\mathrm{MSE}, F, v, p, 0}\right)} \stackrel{p}{\rightarrow} 1
$$

Proof: Because the IK bandwidth optimal for estimating $\tau_{Y, V}$ shrinks at the rate of $n^{-\frac{1}{2 p+3}}$, the preliminary estimators, $\tilde{\tau}_{Y, v}$ and $\tilde{\tau}_{B, v}$, are consistent. The density, variance and covariance estimators are consistent as argued in Imbens and Kalyanaraman (2012). Since the higher derivative estimators also converge to their 
population counterparts, $\hat{C}_{F, p+1, q, v+q+1} \stackrel{p}{\rightarrow} C_{F, p+1, q, v+q+1}$ and $\hat{C}_{F, v, p, v+p+1} \stackrel{p}{\rightarrow} C_{F, v, p, v+p+1}$, and the results of the proposition follow. 


\section{B.3 Discussion of Ganong and Jaeger (2014)}

If we plot the relationship between baseline earnings and the log time to next job not just locally around the kink points as in Figures 5-8, but globally for the whole range of baseline earnings available in the data, it turns out that this relationship is highly nonlinear. The upper- and lower-left panels in Appendix Figure 4 show the raw data plots, with observations centered at the annual cutoff values for the bottom and top-kink points in the unemployment benefit formula. These graphs clearly show the slope change at the kink points. But over the full range of baseline earnings the relationship appears to follow a u-shape.

We are interested in whether this shape is driven by institutional features of the UI system that are not accounted for in our analysis, or whether it is due to compositional changes in observables along the distribution of baseline earnings. For example, the fraction of low educated or female individuals is higher at low levels of baseline earnings than for high levels. To assess the role of compositional changes, we perform a regression of log time to the next job on all observable characteristics on a 5\% subsample over the full range of baseline earnings. ${ }^{34}$ Then we plot the residuals from predictions based on this regression for the remaining 95\% of observation in the right column of Appendix Figure 4. To account for changing thresholds over time, we center the data around the annual bottom and top kink threshold levels. Appendix Figure 4 shows that most of the curvature in the global relationship can be explained by changes in observable characteristics. The residual plots are mostly flat along the range of baseline earnings, and the nonlinearity at the kink points becomes even more salient.

Ganong and Jäger (2014) raise concerns about the sensitivity of the RKD estimates when the relationship between the running variable and the outcome is highly nonlinear. To assess this sensitivity, they suggest a permutation test which shifts the threshold value for the kink across the support of the running variable and estimates slope changes in these placebo samples. One example to demonstrate their method is based on the raw data plot for the bottom kink sample in Appendix Figure 4. Ganong and Jäger (2014) argue that because of the curvature in the global relationships many of the placebo estimates result in large and significant slope changes.

We replicate this exercise based on the raw individual level data. Following Ganong and Jäger (2014), we randomly draw 200 cutoff values for both the bottom and top kink samples over the range of baseline

\footnotetext{
${ }^{34}$ The list of covariates includes: indicator variables for gender, marital status, Austrian citizenship, education (6 categories), deciles for age, tenure, size, and recall rate at the firm of the last job, monthly wage last job (quintiles), days worked over the last 5 years (quartiles), 8 industry groups interacted with blue collar status, region, year, and month dummies.
} 
earning where there is no kink in the benefit formula. The empirical c.d.f.'s of the coefficient estimate and the corresponding $t$-statistic are shown by the solid lines in Appendix Figure 5, where the graphs in the top row correspond to the bottom kink sample and those in the bottom row correspond to the top kink sample. ${ }^{35}$ The c.d.f. of the placebo coefficient estimate is similar to Figure 3 in Ganong and Jäger (2014), although the median value is somewhat lower in our sample. But the placebo estimates find a high share of coefficient estimates that are significantly different from zero. The same pattern appears for the top kink sample, although in this case the sign of the placebo estimates is the opposite of that of the reduced form RKD estimate in Table 2.

Since the curvature in the global relationship can be explained by observable characteristics, we repeat the permutation test on the residuals. The corresponding c.d.f.'s of the placebo coefficient estimate and $t$ statistic are plotted using the dashed lines in the graphs in Appendix Figure 5. The placebo point estimates now have a high density around zero, and the absolute values of the $t$-statistics are below 2 .

Our exercise highlights the importance of the role of curvature heterogeneity in the permutation test of Ganong and Jäger (2014). Specifically, the test may not be informative when the curvature of the conditional expectation function $\mu(v) \equiv E[Y \mid V=v]$ changes. Suppose $\mu(v)$ is locally piece-wise linear around the threshold $v=0$ with a non-zero kink but has substantial curvature far away from the threshold, as appears to be true in our data. In this case, the permutation test may fail to detect the kink at the threshold, as the curvature away from the threshold can lead to many significant local linear estimates. Based on a similar argument, the permutation test may also have poor size control when the curvature of $\mu(v)$ changes. Hence, if researchers wish to conduct the permutation test, it will be important to control for confounding nonlinearities by taking the distribution of observables into account, as pointed out by Ando (2014). We conclude that after controlling for covariates our design passes the Ganong and Jäger (2014) permutation test.

In addition to the permutation test, Ganong and Jäger (2014) also assess the performance of various estimators in two DGP's based on sine functions. Relying upon their simulation results, they advise practitioners to use either 1) what we call the bias-corrected local quadratic estimator with the default CCT bandwidth or 2) cubic spline with the generalized cross validation bandwidth. In an additional simulation study, we have modified the Ganong and Jäger (2014) DGP's slightly, ${ }^{36}$ yielding a Monte Carlo exercise

\footnotetext{
${ }^{35}$ We estimate local linear models with a fixed bandwidth of 2500 for the bottom kink sample and a fixed bandwidth of 4000 for the top kink sample.

${ }^{36}$ The DGP's in Ganong and Jäger (2014) are $\mu(v)=10 x \times 1_{[x>0]}+\sin (k(v-0.1))+v^{2}+\varepsilon$ where $k=5,15$. In our DGP, we set
} 
in which the robust confidence interval for the local quadratic estimator with the default CCT bandwidth selector has a much lower coverage rate (13.9\%) than its local linear counterpart $(80.4 \%) .{ }^{37}$ Despite the inferior performance of the bias-corrected local quadratic estimator in this particular DGP, we do not argue that a local quadratic estimator should never be used. Rather, we believe that caution is needed in attempting to draw practical advice from specific Monte Carlo studies, and suggest instead that researchers use a combination of methods - including simulations studies based on DGP's that closely resemble their actual data - to determine a preferred estimator (or set of estimators) for their particular setting.

$k=25$.

${ }^{37}$ In each repetition, we compute the main and the pilot CCT bandwidths and the robust confidence interval using the nearestneighbor variance estimator. The lower coverage rates of the local quadratic confidence interval is a result of the larger CCT main bandwidth (0.14) as compared to its linear counterpart (0.04). 


\section{A Job Search Model with Wage-Dependent UI Benefits}

This Appendix describes an equilibrium wage posting model with a wage-dependent UI benefit, and a maximum benefit level. We ask to what extent the model is consistent the RKD identifying assumptions, and reach two main conclusions. First, when there is a kink in the UI benefit formula, a baseline model predicts a kink in the density of wages among job losers at the level of wages corresponding to the maximum benefit. Second, this prediction relies on complete information about the location of the kink in the benefit schedule and is not robust to allowing for small errors in agents' beliefs about the location of the kink in the benefit schedule.

Setup. Consider an infinite horizon, discrete-time, posted-wage model of job search with an exogenous distribution of wage offers, and equally efficient search among employed and unemployed agents. With a level of search intensity $s$, the arrival rate of job offers is $\lambda \cdot s$; there is also an exogenous job destruction rate of $\delta$. There is a strictly increasing and convex cost-of-search function $c(\cdot)$ with $c(0)=0$. Wage offers come from a stationary, twice continuously differentiable c.d.f. $F(\cdot)$. The setup is identical to the model used by Christensen et al. (2005), except that we cast the problem in discrete time (with a discount rate $\beta$ ) and assume a wage-dependent UI benefit. ${ }^{38}$

Specifically, we assume that the UI benefit $b$ is a function of the last wage received before being laid off, $w_{-1}$, given by the formula $b\left(w_{-1}\right) \equiv \underline{b}+\rho \min \left(w_{-1}, T^{\max }\right)$, where $\rho<1$ and $b(w)<w$ for all $w$. As in most actual benefit systems, agents with a previous wage above the threshold $T^{\max }$ receive a maximum benefit level $\bar{b}=\underline{b}+\rho T^{\max }$. The dependence of benefits on previous wages adds two novel considerations to the standard search model: 1) when choosing search intensity and whether to accept a wage offer, an agent must take into account the effect of the wage on future UI benefits; 2) when taking a new job, an unemployed worker resets his or her benefit level. Because we assume that UI benefits last indefinitely, and that the benefit is reset immediately upon taking a new job, our model arguably overemphasizes both these considerations relative to a more realistic setting where benefits can expire, and UI entitlement is based on earnings over a previous base period of several quarters' duration.

An agent's choice problem is characterized by two value functions: $W_{e m}(w)$, the value function for being

\footnotetext{
${ }^{38}$ To translate the model to our generalized regression setting, note that we can allow for unrestricted heterogeneity and index all the model's elements by $U$, the unobserved type. The discussion below is conditional on the type $U$, and we suppress any notation indicating the value of $U$.
} 
employed with current wage $w$, and $W_{u n}\left(w_{-1}\right)$, for being unemployed with previous wage $w_{-1}$ :

$$
\begin{gathered}
W_{e m}(w)=\max _{s \geq 0}\left\{w-c(s)+\beta\left[(1-\delta)\left[\lambda s \int \max \left\{W_{e m}(x), W_{e m}(w)\right\} d F(x)+(1-\lambda s) W_{e m}(w)\right]+\delta W_{u n}(w)\right]\right\} \\
W_{u n}\left(w_{-1}\right)=\max _{s \geq 0}\left\{b\left(w_{-1}\right)-c(s)+\beta\left[\lambda s \int \max \left\{W_{e m}(x), W_{u n}\left(w_{-1}\right)\right\} d F(x)+(1-\lambda s) W_{u n}\left(w_{-1}\right)\right]\right\} .
\end{gathered}
$$

Note that $W_{u n}$ is an increasing function of the previous wage for $w_{-1}<T^{\max }$, since a higher previous wage entitles the agent to higher benefits. Once the previous wage reaches the threshold $T^{\text {max }}$, however, there is no further increase in $W_{u n}$ : thus the value function $W_{u n}$ is kinked at $w_{-1}=T^{\max }$, with $W_{u n}^{\prime}\left(w_{-1}\right)>0$ for $w_{-1}<T_{\max }$ and $W_{u n}^{\prime}\left(w_{-1}\right)=0$ for $w_{-1}>T^{\max }$. Inspection of the value functions shows that this in turn induces a kink in $W_{e m}(w)$ at $w=T^{\max }$, provided that $\delta>0$.

Optimal Search Behavior. It can be shown that the optimal behavior is characterized by a reservation wage strategy while employed, another reservation wage strategy while unemployed, and a choice of optimal search intensity $s_{e m}(w)$ when employed at wage $w$ and $s_{u n}\left(w_{-1}\right)$ when unemployed with previous wage $w_{-1} \cdot{ }^{39}$ Clearly, an employed worker will accept any wage offer that exceeds her current wage. An unemployed worker with previous wage $w_{-1}$ will accept any wage offer $w$ with $W_{e m}(w) \geq W_{u n}\left(w_{-1}\right)$, implying a reservation wage $R\left(w_{-1}\right)$ so that $W_{e m}\left(R\left(w_{-1}\right)\right)=W_{u m}\left(w_{-1}\right)$.

It is well known that when the UI benefit is a fixed constant $b$ the optimal strategy for an unemployed worker is to take any job with $w \geq b$, implying $R\left(w_{-1}\right)=b$, since there is no extra disutility of work versus unemployment, and the arrival rate and search costs are the same whether working or not. This simple rule is no longer true when the benefits depend on $w_{-1}$. Consider an unemployed worker with an offer $w=b\left(w_{-1}\right)$. Taking the job will yield the same flow utility as remaining on unemployment, but when the job ends she will receive a lower future UI benefit (assuming that the benefit-replacement rate is less than 1). Thus, a higher wage offer is required for indifference, implying that $R\left(w_{-1}\right)>b\left(w_{-1}\right)$ when $w_{-1}<T^{\max }$.

Given the value functions above, and a strictly increasing, convex, and twice continuously differentiable cost function $c(\cdot)$, we can implicitly solve for the optimal search functions $s_{u n}(\cdot)$ and $s_{e m}(\cdot)$ via the first

\footnotetext{
${ }^{39}$ It can be shown that $W_{e m}(w)$ is strictly increasing in $w$ and that $W_{u n}\left(w_{-1}\right)$ is increasing in $w_{-1}$, which leads to reservation wage strategies in each case.
} 
order conditions for interior solutions for (17) and (18),

$$
\begin{aligned}
c^{\prime}\left(s_{e m}(w)\right) & =\beta(1-\delta) \lambda \int_{w}^{\bar{w}}\left[W_{e m}(x)-W_{e m}(w)\right] d F(x) \\
c^{\prime}\left(s_{u n}\left(w_{-1}\right)\right) & =\beta \lambda \int_{R\left(w_{-1}\right)}^{\bar{w}}\left[W_{e m}(x)-W_{u n}\left(w_{-1}\right)\right] d F(x),
\end{aligned}
$$

where $\bar{w}$ is the upper bound of the support of the offer distribution. Consideration of these first order conditions shows that the optimal levels of search intensity both have a kink at the wage threshold $T^{\max }$. For example, the right derivative of $s_{e m}(w)$ at $w=T^{\max }$ is

$$
s_{e m}^{\prime}\left(T^{\max +}\right)=\frac{-\beta(1-\delta) \lambda(1-F(T))}{c^{\prime \prime}\left(s_{e m}(T)\right)} W_{e m}^{\prime}\left(T^{\max +}\right)
$$

while the derivative from the left is

$$
s_{e m}^{\prime}\left(T^{\max -}\right)=\frac{-\beta(1-\delta) \lambda(1-F(T))}{c^{\prime \prime}\left(s_{e m}(T)\right)} W_{e m}^{\prime}\left(T^{\max -}\right) .
$$

Since $W_{e m}(w)$ has a kink at $w=T^{\max }, W^{\prime}\left(T^{\max +}\right) \neq W^{\prime}\left(T^{\max -}\right)$ and the left and right limits of the derivative of $s_{e m}(w)$ are different at $w=T^{\max }$. A similar argument applies to the derivative of $s_{u n}\left(w_{-1}\right)$ at $w_{-1}=T^{\max }$.

Steady State Wage Distribution. A standard wage posting model yields a steady state unemployment rate $u$ and a steady state distribution of wages $G(w)$ that stochastically dominates the distribution of wage offers $F(w)$, reflecting the fact that employed workers are always searching for higher wage offers. When the benefit level varies across unemployed workers, and workers with different benefit levels have different reservation wages, there is also a steady state distribution of previous wages in the stock of unemployed workers, which we denote by $H(w) .{ }^{40}$

In the steady state, the inflow into the set of workers employed with a wage of $w$ or less must equal the outflow:

$$
\begin{aligned}
u \lambda \int_{0}^{\bar{w}} s_{\text {un }}(x)[\max \{F(w)-F(R(x)), 0\}] d H(x) & =\delta G(w)(1-u)+\left[(1-\delta) \lambda(1-u) \int_{0}^{w} s_{\text {em }}(x) d G(x)\right](1-F(w)) \\
\operatorname{Inflow}(w) & =\operatorname{Layoff}(w)+\operatorname{Offer}(w) \cdot(1-F(w)) .
\end{aligned}
$$

\footnotetext{
${ }^{40}$ It can be shown that:

$$
H(w)=\frac{\int_{0}^{w} \frac{d G(x)}{\lambda s_{u n}(x)-\lambda s_{u n}(x) F(R(x))}}{\int_{0}^{\bar{w}} \frac{d G(x)}{\lambda s_{u n}(x)-\lambda s_{u n}(x) F(R(x))}} .
$$
}


The quantity $\operatorname{Inflow}(w)$ is the fraction of the stock of unemployed workers who receive a wage offer that exceeds their reservation wage, but is less than $w$. On the right hand side, the proportion with a wage less than $w$ and displaced with probability $\delta$ is given by Layoff $(w)$ ), while the proportion of individuals who will leave jobs that pay less than $w$ for jobs that pay more than $w$ is given by $(\operatorname{Offer}(w) \cdot(1-F(w)))$.

Now consider a $w$ within a neighborhood of the threshold $T^{\max } \cdot{ }^{41}$ Consider the above flow equation for observed wages between $w+h$ and $w$. Some rearrangement yields:

$$
\begin{aligned}
\operatorname{Inflow}(w+h)-\operatorname{Inflow}(w)+\text { Offer }(w)((F(w+h))-F(w))= & \text { Layoff }(w+h)-\operatorname{Layoff}(w) \\
& +(\text { Offer }(w+h)-\text { Offer }(w))(1-F(w+h)) .
\end{aligned}
$$

Applying a mean value theorem for Stieltjes integrals on the right hand side, rearranging, and dividing by $h$, we obtain

$$
\frac{\frac{\operatorname{Inflow}(w+h)-\operatorname{Inflow}(w)}{h}+O f f e r(w) \frac{F(w+h)-F(w)}{h}}{\delta(1-u)+(1-\delta) \lambda(1-u) c_{O}(1-F(w+h))}=\frac{G(w+h)-G(w)}{h}
$$

where $\inf _{x \in[w, w+h]} S_{e m}(x) \leq c_{O} \leq \sup _{x \in[w, w+h]} S_{e m}(x)$.

By assumption, the distribution of wage offers $F(\cdot)$ is differentiable. Moreover, it can be shown that the search intensity choice of employed workers is continuous, and that $\operatorname{Inflow}(w)$ is differentiable in a neighborhood of $T .{ }^{42}$ Taking the limit as $h \rightarrow 0$, we obtain:

$$
\frac{\operatorname{Inflow}^{\prime}(w)+\text { Offer }(w) f(w)}{\left(\delta(1-u)+(1-\delta) \lambda(1-u) s_{\text {em }}(w)(1-F(w))\right)}=g(w)
$$

which means that the density of wages $g(w)$ is well-defined in this neighborhood.

It can be shown that every function of $w$ on the left-hand side of this equation is continuously differentiable at $w=T^{\max }$ except the search intensity function $s_{e m}(\cdot)$, which is kinked at $T^{\max }$. As noted above, this arises because of the kinks in the value functions $W_{e m}(\cdot)$ and $W_{u m}(\cdot)$ at the wage threshold $T^{\max }$. As a consequence, the density of wages among employed workers has a kink at $w=T^{\max }$. Assuming that the job destruction rate is constant across all jobs, the population of new UI claimants has the same distribution of previous wages as the pool of employed workers. As a consequence, this model implies that the density of

\footnotetext{
${ }^{41}$ We choose a neighborhood of $T^{\max }$ in which $w>R\left(T^{\max }\right)$. Such a neighborhood always exists because $T^{\max }>R\left(T^{\max }\right)-\mathrm{a}$ worker who accepts a wage $T^{\max }$ will be strictly better off than remaining unemployed with the maximum benefit $\bar{b}$.

${ }^{42}$ Differentiability of $\operatorname{Inflow}(\cdot)$ follows because in a neighborhood of $T, w>R(x)$ for all $x$. Thus Inflow (w) $=$ $u \lambda \int_{0}^{\bar{w}} s_{u n}(x)[F(w)-F(R(x))] d H(x)$. We can differentiate under the integral sign because the derivative of the integrand with respect to $w$ is continuous in the rectangle defined by the neighborhood of $T$ and $[0, \bar{w}]$, and $F(\cdot)$ is differentiable by assumption.
} 
wages among new UI claimants has a kink at $w=T^{\max }$.

Model with Imperfect Information About Benefit Schedules. We now consider a variant of the preceding model in which agents have imperfect information on the location of the kink point in the benefit schedules. We show that the prediction of a kinked density is not robust to small errors. To proceed, assume that the true kink in the benefit schedule occurs at $w=T^{\max }$, but the agent makes choices assuming the kink is at $T^{\max }+\varepsilon$. This leads to value functions, indexed by the error $\varepsilon, W_{e m}^{\varepsilon}(w)$ and $W_{u n}^{\varepsilon}(w)$ parallel to those in equations (17) and (18). In addition, there is another value function defined by

$$
W_{u n}^{\varepsilon *}\left(w_{-1}\right)=\max _{s \geq 0}\left\{b\left(w_{-1}\right)-c(s)+\beta\left[\lambda s \int \max \left\{W_{e m}^{\varepsilon}(x), W_{u n}^{\varepsilon *}\left(w_{-1}\right)\right\} d F(x)+(1-\lambda s) W_{u n}^{\varepsilon *}\left(w_{-1}\right)\right]\right\} .
$$

$W_{u n}^{\varepsilon}\left(w_{-1}\right)$ is the perceived value of unemployment for a worker using an incorrect benefit formula, whereas $W_{u n}^{\varepsilon *}\left(w_{-1}\right)$ is the perceived value of unemployment of an unemployed worker who is receiving benefits $b\left(w_{-1}\right)$ based on the correct formula, but is evaluating the value of potential future employment using $W_{e m}^{\varepsilon}$.

The result of this small optimization error is that actual search intensity for an individual (in the employed and unemployed state) will be given by the first-order conditions

$$
\begin{aligned}
c^{\prime}\left(s_{e m}^{\varepsilon}(w)\right) & =\beta(1-\delta) \lambda \int_{w}^{\bar{w}}\left[W_{e m}^{\varepsilon}(x)-W_{e m}^{\varepsilon}(w)\right] d F(x) \\
c^{\prime}\left(s_{u n}^{\varepsilon *}\left(w_{-1}\right)\right) & =\beta \lambda \int_{R^{\varepsilon *}\left(w_{-1}\right)}^{\bar{w}}\left[W_{e m}^{\varepsilon}(x)-W_{u n}^{\varepsilon *}\left(w_{-1}\right)\right] d F(x) .
\end{aligned}
$$

Moreover, the reservation wage for employed agents is still their current wage, while the reservation wage if unemployed is $R^{\varepsilon *}\left(w_{-1}\right)$, implicitly defined by $W_{e m}^{\varepsilon}\left(R^{\varepsilon *}\left(w_{-1}\right)\right)=W_{u n}^{\varepsilon *}\left(w_{-1}\right)$.

With an error in the perceived kink, the steady state flow equation for the wage density $G(w)$ is the same as in equation (20), after replacing $s_{e m}(\cdot), s_{u n}(\cdot)$ with $s_{e m}^{\varepsilon}(\cdot), s_{u n}^{\varepsilon *}$. As a result, the steady state density for a population of agents of type " $\varepsilon$ " exhibits a kink at $T^{\max }+\varepsilon$. If the true population contains a mixture of agents with different values of $\varepsilon$, drawn from a density $\phi_{\varepsilon}(\cdot)$, then the steady state flow equation for the density of wages is the same as in equation (20), after replacing $s_{e m}(\cdot), s_{u n}(\cdot)$ with $E\left[s_{e m}^{\varepsilon}(\cdot)\right], E\left[s_{u n}^{\mathcal{E} *}(\cdot)\right]$ where expectations are taken with respect to $\phi_{\varepsilon}(\cdot)$. It can be shown that if $\varepsilon$ is continuously distributed, then $E\left[s_{e m}^{\varepsilon}(x)\right]$ will be continuously differentiable, leading to a continuously differentiable steady state density $g(\cdot) .{ }^{43}$ Thus, a continuous distribution of errors in agents' beliefs about the location of the kink point will smooth out the kink that arises with full information.

\footnotetext{
${ }^{43}$ Specifically, $E\left[s_{e m}^{\varepsilon}(w)\right]$ is continuously differentiable because $\frac{d s_{e m}^{\varepsilon}(w)}{d w}$ is continuous at $w=T^{\max }$ almost everywhere (it is non-differentiable only when $\varepsilon=0$, a measure zero event).
} 

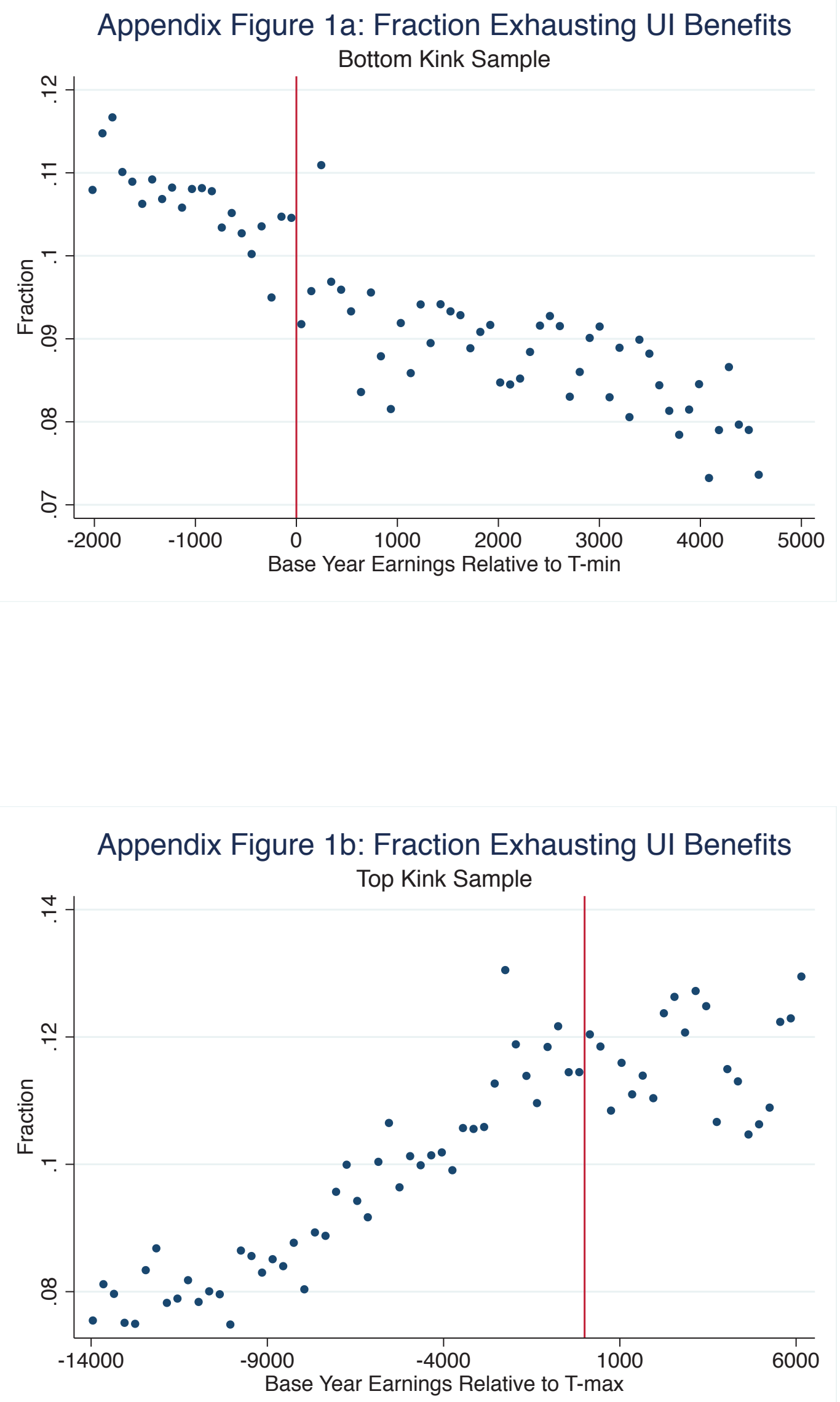


\section{Appendix Figure 2: Covariates Bottom Kink Sample}
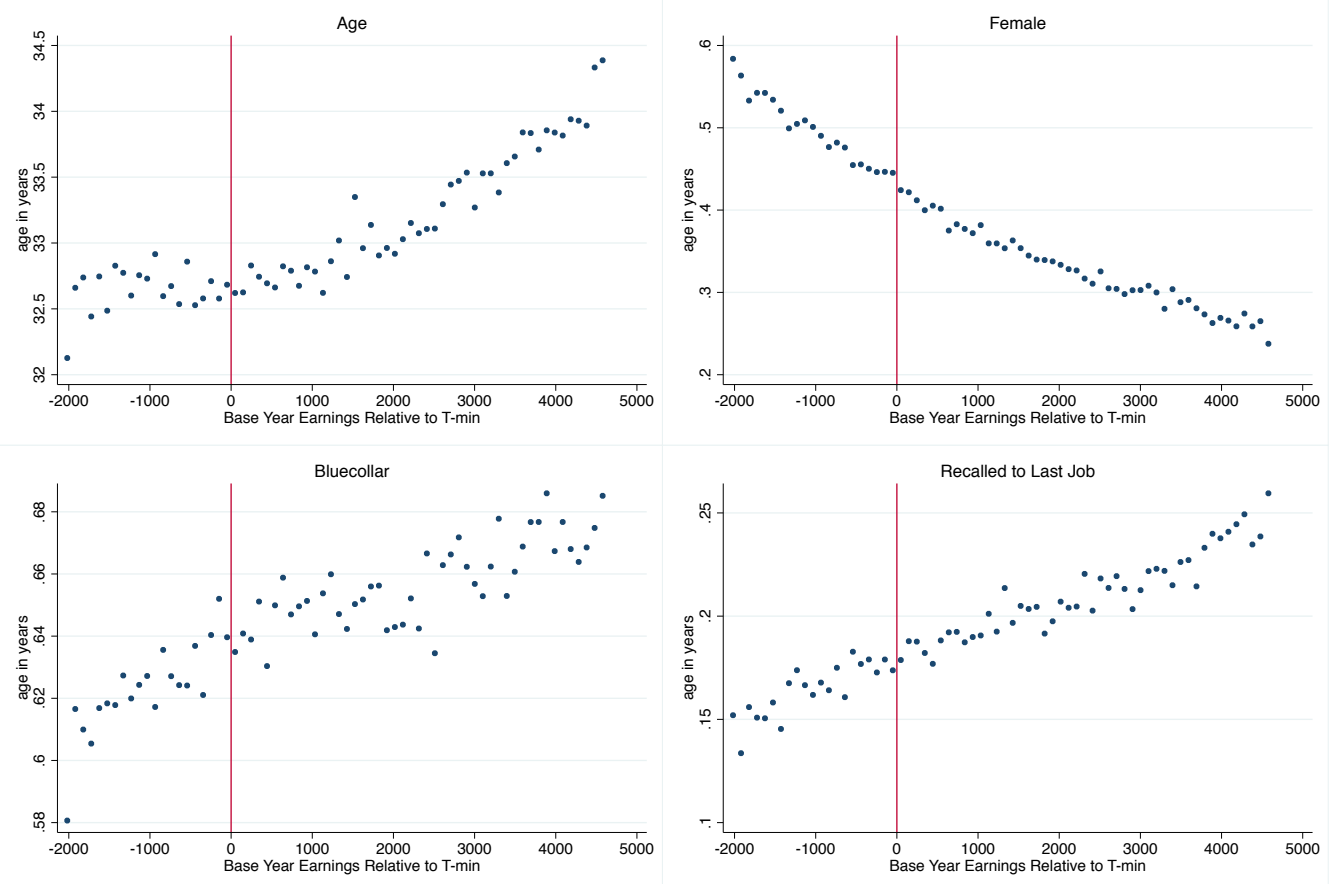


\section{Appendix Figure 3: Covariates Top Kink Sample}
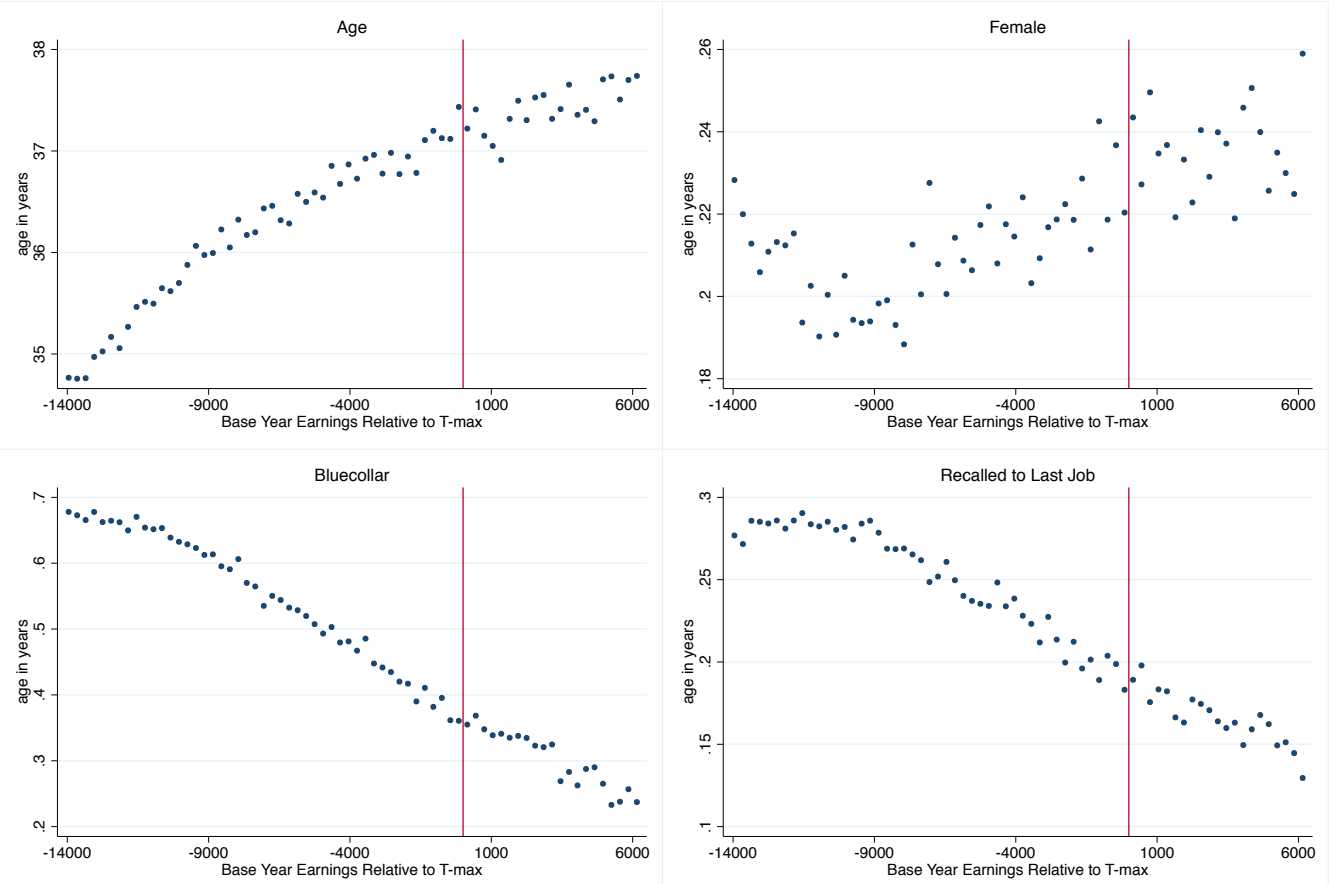
Appendix Figure 4: Global Relationship between Log Time to Next Job and Baseline Earnings
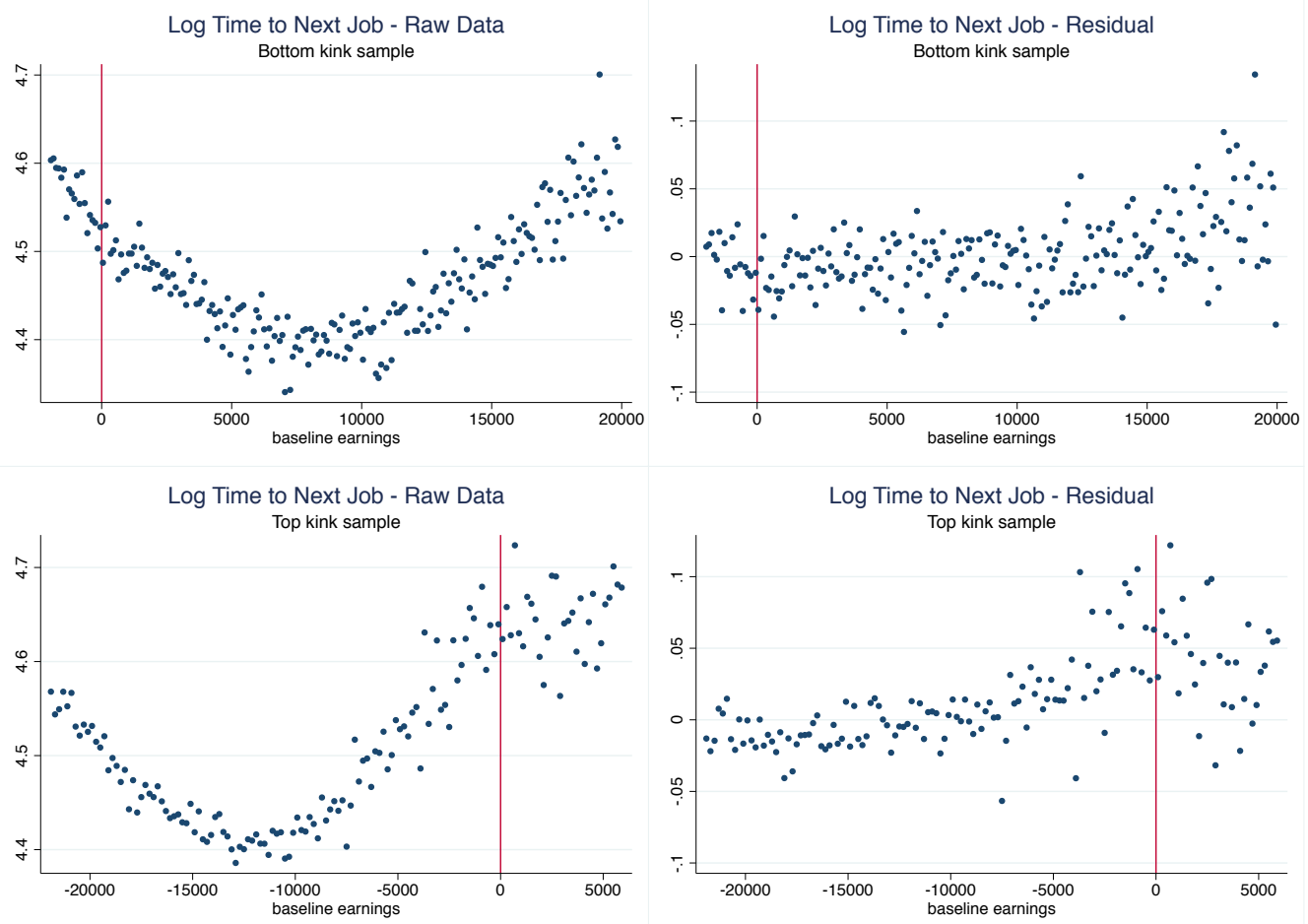
Appendix Figure 5: Distribution of the Coefficient Estimate and t-Statistic in the Permutation Test
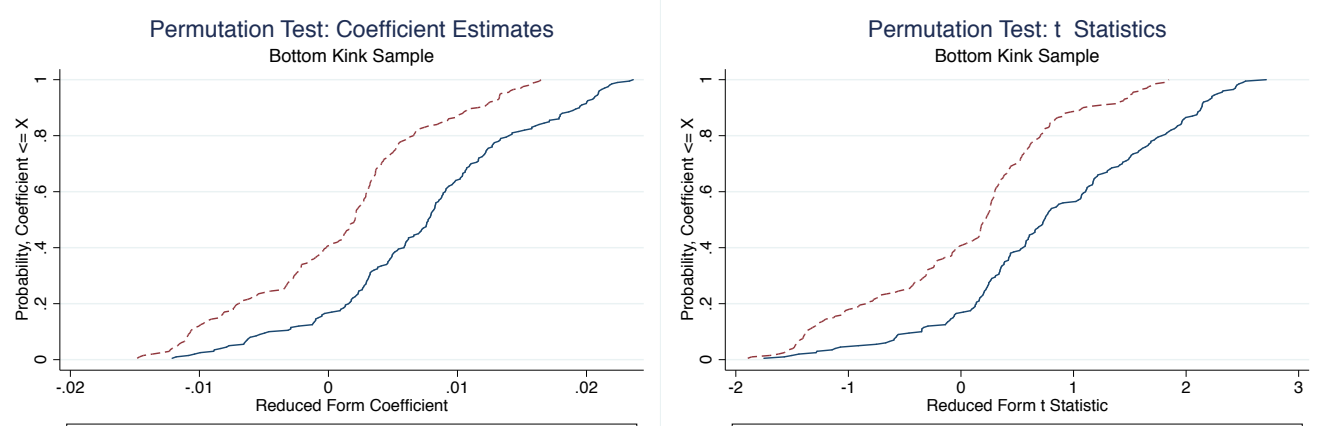

CDF Estimates Raw Data - - - - CDF Estimates Residual
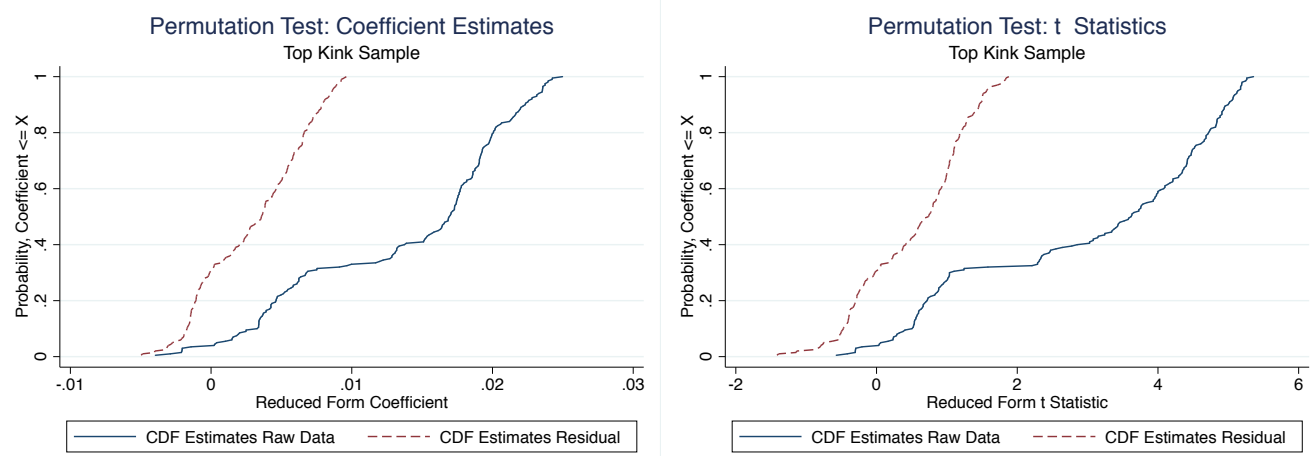
Appendix Table 1: Testing for Smooth Density of Previous Earnings Using Parametric Models of Frequency Distributions in Bottom Kink and Top Kink Samples

\begin{tabular}{|c|c|c|c|}
\hline $\begin{array}{l}\text { Polynomial Order of } \\
\text { Model for Histogram } \\
\text { (1) }\end{array}$ & $\begin{array}{c}\text { Estimated Kink x } 1000 \\
\text { (Standard Error) } \\
(2)\end{array}$ & $\begin{array}{c}\text { Pearson } \chi^{2} \\
\text { (P-value) } \\
\text { (3) }\end{array}$ & $\begin{array}{c}\text { Akaike } \\
\text { Criterion } \\
\text { (4) }\end{array}$ \\
\hline \multicolumn{4}{|c|}{ A. Bottom Kink Sample ( 65 bins of width $=100$ Euro/year) } \\
\hline 2 & $\begin{array}{c}0.446 \\
(0.172)\end{array}$ & $\begin{array}{l}83.02 \\
(2.6 \%)\end{array}$ & 93.02 \\
\hline 3 & $\begin{array}{l}-0.080 \\
(0.091)\end{array}$ & $\begin{array}{l}73.81 \\
(7.9 \%)\end{array}$ & 87.81 \\
\hline 4 & $\begin{array}{l}-0.240 \\
(0.181)\end{array}$ & $\begin{array}{l}72.43 \\
(6.9 \%)\end{array}$ & 90.43 \\
\hline 5 & $\begin{array}{c}0.109 \\
(0.308)\end{array}$ & $\begin{array}{l}69.39 \\
(7.7 \%)\end{array}$ & 91.39 \\
\hline \multicolumn{4}{|c|}{ B. Top Kink Sample (66 bins of width $=300$ Euro/year) } \\
\hline 2 & $\begin{array}{l}-0.070 \\
(0.035)\end{array}$ & $\begin{array}{l}142.55 \\
(0.0 \%)\end{array}$ & 152.55 \\
\hline 3 & $\begin{array}{l}-0.220 \\
(0.081)\end{array}$ & $\begin{array}{l}124.15 \\
(0.0 \%)\end{array}$ & 138.15 \\
\hline 4 & $\begin{array}{c}0.200 \\
(0.124)\end{array}$ & $\begin{array}{l}75.62 \\
(5.0 \%)\end{array}$ & 93.62 \\
\hline 5 & $\begin{array}{c}0.158 \\
(0.213)\end{array}$ & $\begin{array}{c}75.50 \\
(3.5 \%)\end{array}$ & 97.50 \\
\hline
\end{tabular}

Notes: See text. Bottom kink sample includes 254,489 observations (2,803 observations in left-most and rightmost bins deleted). Top kink sample includes 271,277 observations (4,388 observations in left-most and rightmost bins deleted). Models are estimated by minimum chi-square. Model for fraction of observations in a bin is a polynomial function (of the order indicated in column 1) of the bin counter, with interactions of poynomial terms with indicator for bins to the right of the kink point (main effect of indicator is excluded). Estimated kink in column 2 is coefficient of interaction between linear term and indicator for bins to right of kink point. Akaike criterion in column 4 is chi-squared model fit statistic plus 2 times the number of parameters in the model. 
Appendix Table 2a: Summary of Monte Carlo Studies, DGP Design Based on Bottom Kink Sample

\begin{tabular}{|c|c|c|c|c|c|c|c|c|c|c|c|}
\hline & \multirow[b]{2}{*}{$\begin{array}{c}\text { Median } \\
\text { Main b.w. } \\
\text { (1) }\end{array}$} & \multirow[b]{2}{*}{$\begin{array}{c}\text { Median } \\
\text { Pilot b.w. } \\
\text { (2) }\end{array}$} & \multicolumn{3}{|c|}{ First Stage Model Estimation Summary } & \multicolumn{6}{|c|}{ Elasticity Estimation Summary } \\
\hline & & & $\begin{array}{c}\text { Fraction of } \\
\text { Replications: C.I. } \\
\text { includes } 0 \\
\text { (3) }\end{array}$ & $\begin{array}{l}\text { RMSE/true } \\
\text { value } \\
\text { (trimmed) } \\
(4) \\
\end{array}$ & $\begin{array}{c}\text { C.I. } \\
\text { Coverage } \\
\text { Rate } \\
(5) \\
\end{array}$ & $\begin{array}{l}\text { RMSE } \\
(6) \\
\end{array}$ & $\begin{array}{c}\text { RMSE } \\
\text { (trimmed) } \\
(7)\end{array}$ & $\begin{array}{l}\text { C.I. } \\
\text { Coverage } \\
\text { Rate } \\
\text { (8) }\end{array}$ & $\begin{array}{c}\text { Bias } \\
\text { (trimmed) } \\
\text { (9) }\end{array}$ & $\begin{array}{c}\text { Bias }^{2} \\
\text { (trimmed) } \\
(10)\end{array}$ & $\begin{array}{c}\begin{array}{c}\text { Variance } \\
\text { (trimmed) }\end{array} \\
\text { (11) }\end{array}$ \\
\hline \multicolumn{12}{|c|}{ 1. Local Linear, No Bias Correction } \\
\hline Default CCT & 470 & - & 0.31 & 0.43 & 0.94 & 20.3 & 4.81 & 1.00 & 0.72 & 0.52 & 22.6 \\
\hline $\mathrm{CCT}$, no regularization & 998 & - & 0.04 & 0.22 & 0.89 & 4.7 & 2.04 & 0.77 & 0.68 & 0.46 & 3.69 \\
\hline Fuzzy CCT & 973 & - & 0.04 & 0.22 & 0.89 & 22.3 & 1.90 & 0.77 & 0.75 & 0.56 & 3.06 \\
\hline Fuzzy IK & 1,415 & - & 0.00 & 0.11 & 0.85 & 1.60 & 1.30 & 0.55 & 0.83 & 0.69 & 1.01 \\
\hline FG & 2,600 & - & 0.00 & 0.08 & 0.60 & 1.40 & 1.35 & 0.02 & 1.32 & 1.75 & 0.09 \\
\hline Global (all data) & 4,564 & - & 0.00 & 0.14 & 0.00 & 1.29 & 1.25 & 0.01 & 1.22 & 1.49 & 0.07 \\
\hline \multicolumn{12}{|c|}{ 2. Local Linear, Bias-Corrected } \\
\hline Default CCT & 470 & 970 & 0.60 & 0.62 & 0.94 & $7.0 \mathrm{E}+02$ & 8.5 & 1.00 & 0.71 & 0.51 & 71.1 \\
\hline $\mathrm{CCT}$, no regularization & 998 & 1,311 & 0.33 & 0.47 & 0.92 & 10.6 & 3.96 & 0.97 & 0.38 & 0.14 & 15.6 \\
\hline Fuzzy CCT & 973 & 1,363 & 0.29 & 0.45 & 0.92 & $1.2 \mathrm{E}+03$ & 3.76 & 0.98 & 0.39 & 0.16 & 14.0 \\
\hline Fuzzy IK & 1,415 & 1,615 & 0.21 & 0.36 & 0.90 & 3.84 & 2.96 & 0.92 & 0.31 & 0.10 & 8.70 \\
\hline FG & 2,600 & 3,367 & 0.00 & 0.16 & 0.89 & 1.89 & 1.69 & 0.82 & 0.87 & 0.75 & 2.10 \\
\hline Global (all data) & 4,564 & 4,564 & 0.00 & 0.18 & 0.83 & 2.17 & 1.97 & 0.75 & 1.54 & 2.36 & 1.54 \\
\hline \multicolumn{12}{|c|}{ 3. Quadratic, No Bias Correction } \\
\hline Default CCT & 681 & - & 0.78 & 0.93 & 0.95 & 540 & 13.5 & 1.00 & -0.38 & 0.15 & 183 \\
\hline $\mathrm{CCT}$, no regularization & 1,211 & - & 0.38 & 0.50 & 0.91 & 84 & 6.6 & 0.98 & 0.41 & 0.16 & 43.2 \\
\hline Fuzzy CCT & 1,291 & - & 0.35 & 0.47 & 0.91 & 67 & 5.6 & 0.98 & 0.57 & 0.32 & 31.1 \\
\hline Fuzzy IK & 1,778 & - & 0.09 & 0.27 & 0.90 & 135 & 2.77 & 0.95 & 0.39 & 0.15 & 7.55 \\
\hline FG & 3,718 & - & 0.00 & 0.14 & 0.88 & 2.12 & 1.90 & 0.72 & 1.27 & 1.60 & 2.00 \\
\hline Global (all data) & 4,564 & - & 0.00 & 0.17 & 0.76 & 2.56 & 2.39 & 0.54 & 2.13 & 4.54 & 1.15 \\
\hline \multicolumn{12}{|c|}{ 4. Local Quadratic, Bias-Corrected } \\
\hline Default CCT & 681 & 1,079 & 0.85 & 1.25 & 0.95 & 703252 & 22.4 & 1.00 & 1.03 & 1.07 & 499 \\
\hline $\mathrm{CCT}$, no regularization & 1,211 & 1,384 & 0.76 & 1.02 & 0.92 & $2.2 \mathrm{E}+04$ & 13.2 & 1.00 & 0.48 & 0.23 & 175 \\
\hline Fuzzy CCT & 1,291 & 1,462 & 0.76 & 1.00 & 0.94 & 4058 & 12.8 & 0.99 & 0.38 & 0.15 & 165 \\
\hline Fuzzy IK & 1,778 & 1,657 & 0.73 & 1.21 & 0.92 & $5.2 E+05$ & 10.6 & 0.96 & 0.55 & 0.30 & 113 \\
\hline FG & 3,718 & 4,945 & 0.24 & 0.37 & 0.90 & 3.54 & 3.00 & 0.95 & -0.01 & 0.00 & 9.03 \\
\hline Global (all data) & 4,564 & 4,564 & 0.24 & 0.37 & 0.89 & 3.67 & 3.11 & 0.95 & -0.01 & 0.00 & 9.70 \\
\hline
\end{tabular}

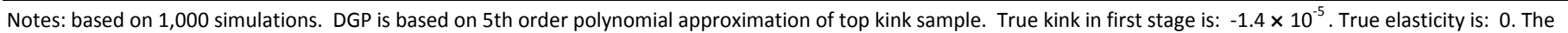
trimmed statistic are obtained by first trimming the $5 \%$ sample in which the estimates deviate the most from the true parameter value. 
Appendix Table 2b: Summary of Monte Carlo Studies, DGP Design Based on Top Kink Sample

\begin{tabular}{|c|c|c|c|c|c|c|c|c|c|c|c|}
\hline & \multirow[b]{2}{*}{$\begin{array}{c}\text { Median } \\
\text { Main b.w. } \\
\text { (1) }\end{array}$} & \multirow[b]{2}{*}{$\begin{array}{c}\text { Median } \\
\text { Pilot b.w. } \\
(2)\end{array}$} & \multicolumn{3}{|c|}{ First Stage Model Estimation Summary } & \multicolumn{6}{|c|}{ Elasticity Estimation Summary } \\
\hline & & & $\begin{array}{c}\text { Fraction of } \\
\text { Replications: C.I. } \\
\text { includes } 0 \\
\text { (3) }\end{array}$ & $\begin{array}{l}\text { RMSE/true } \\
\text { value } \\
\text { (trimmed) } \\
(4) \\
\end{array}$ & $\begin{array}{l}\text { C.I. } \\
\text { Coverage } \\
\text { Rate } \\
(5)\end{array}$ & $\begin{array}{l}\text { RMSE } \\
(6) \\
\end{array}$ & $\begin{array}{c}\text { RMSE } \\
\text { (trimmed) } \\
(7)\end{array}$ & $\begin{array}{l}\text { C.I. } \\
\text { Coverage } \\
\text { Rate } \\
(8) \\
\end{array}$ & $\begin{array}{c}\text { Bias } \\
\text { (trimmed) } \\
(9)\end{array}$ & $\begin{array}{c}\text { Bias }^{2} \\
\text { (trimmed) } \\
(10)\end{array}$ & $\begin{array}{c}\begin{array}{c}\text { Variance } \\
\text { (trimmed) }\end{array} \\
(11)\end{array}$ \\
\hline \multicolumn{12}{|c|}{ 1. Local Linear, No Bias Correction } \\
\hline Default CCT & 1,395 & - & 0.60 & 0.58 & 0.81 & 61.2 & 7.25 & 1.00 & 0.39 & 0.15 & 52.5 \\
\hline $\mathrm{CCT}$, no regularization & 2,810 & - & 0.13 & 0.32 & 0.73 & 29.6 & 2.39 & 0.68 & 0.72 & 0.52 & 5.18 \\
\hline Fuzzy CCT & 2,302 & - & 0.24 & 0.43 & 0.70 & 63.3 & 3.94 & 0.77 & 0.84 & 0.71 & 14.8 \\
\hline Fuzzy IK & 4,396 & - & 0.00 & 0.16 & 0.73 & 1.54 & 1.33 & 0.32 & 1.07 & 1.15 & 0.63 \\
\hline FG & 5,681 & - & 0.00 & 0.18 & 0.64 & 1.31 & 1.23 & 0.20 & 1.11 & 1.23 & 0.27 \\
\hline Global (all data) & 13,908 & - & 0.00 & 0.55 & 0.00 & 0.37 & 0.35 & 0.17 & 0.34 & 0.11 & 0.01 \\
\hline \multicolumn{12}{|c|}{ 2. Local Linear, Bias-Corrected } \\
\hline Default CCT & 1,395 & 2,860 & 0.89 & 0.89 & 0.78 & $2.5 \mathrm{E}+07$ & 20.0 & 0.99 & 0.49 & 0.24 & 400 \\
\hline $\mathrm{CCT}$, no regularization & 2,810 & 3,883 & 0.64 & 0.74 & 0.68 & 1219 & 5.83 & 0.92 & 0.32 & 0.10 & 33.9 \\
\hline Fuzzy CCT & 2,302 & 3,623 & 0.68 & 0.77 & 0.68 & $9.5 \mathrm{E}+05$ & 9.80 & 0.95 & 0.53 & 0.29 & 95.8 \\
\hline Fuzzy IK & 4,396 & 4,796 & 0.43 & 0.61 & 0.69 & 3.74 & 2.80 & 0.85 & 0.62 & 0.38 & 7.44 \\
\hline FG & 5,681 & 9,470 & 0.00 & 0.17 & 0.89 & 2.00 & 1.82 & 0.63 & 1.52 & 2.31 & 1.02 \\
\hline Global (all data) & 13,908 & 13,908 & 0.00 & 0.22 & 0.72 & 2.62 & 2.51 & 0.12 & 2.40 & 5.75 & 0.54 \\
\hline \multicolumn{12}{|c|}{ 3. Quadratic, No Bias Correction } \\
\hline Default CCT & 2,045 & - & 0.90 & 1.08 & 0.89 & 134 & 17.6 & 1.00 & 0.42 & 0.18 & 309 \\
\hline CCT, no regularization & 3,528 & - & 0.63 & 0.75 & 0.74 & 182 & 12.6 & 0.97 & 0.21 & 0.04 & 159 \\
\hline Fuzzy CCT & 3,624 & - & 0.65 & 0.72 & 0.71 & 284 & 11.4 & 0.96 & 0.16 & 0.03 & 131 \\
\hline Fuzzy IK & 5,128 & - & 0.30 & 0.47 & 0.72 & 81.1 & 4.90 & 0.90 & 0.50 & 0.25 & 23.8 \\
\hline FG & 10,455 & - & 0.00 & 0.16 & 0.81 & 3.21 & 3.04 & 0.24 & 2.82 & 7.93 & 1.29 \\
\hline Global (all data) & 13,908 & - & 0.00 & 0.20 & 0.58 & 5.13 & 4.94 & 0.00 & 4.83 & 23.4 & 1.03 \\
\hline \multicolumn{12}{|c|}{ 4. Local Quadratic, Bias-Corrected } \\
\hline Default CCT & 2,045 & 3,234 & 0.92 & 1.37 & 0.90 & $1.8 \mathrm{E}+04$ & 34.2 & 1.00 & 0.26 & 0.07 & 1170 \\
\hline CCT, no regularization & 3,528 & 4,181 & 0.94 & 1.49 & 0.79 & $2.1 \mathrm{E}+07$ & 56.7 & 0.99 & 1.64 & 2.69 & 3217 \\
\hline Fuzzy CCT & 3,624 & 4,262 & 0.92 & 1.37 & 0.79 & 1.7E+05 & 50.7 & 0.98 & -0.21 & 0.05 & 2574 \\
\hline Fuzzy IK & 5,128 & 4,998 & 0.93 & 1.49 & 0.70 & 2552 & 21.6 & 0.93 & 0.37 & 0.14 & 466 \\
\hline FG & 10,455 & 13,218 & 0.72 & 0.65 & 0.60 & 3.65 & 3.04 & 0.93 & 0.68 & 0.46 & 8.81 \\
\hline Global (all data) & 13,908 & 13,908 & 0.79 & 0.71 & 0.49 & 4.47 & 3.67 & 0.91 & 1.52 & 2.30 & 11.2 \\
\hline
\end{tabular}

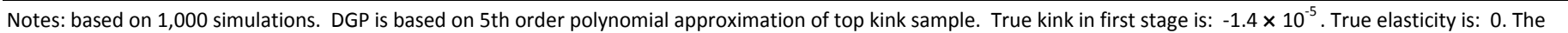
trimmed statistic are obtained by first trimming the $5 \%$ sample in which the estimates deviate the most from the true parameter value. 
Appendix Table 3: Estimated AMSE for Local Linear and Local Quadratic Models

Default CCT bandwidth

\begin{tabular}{|c|c|c|c|c|c|c|}
\hline & \multicolumn{3}{|c|}{ Local Linear } & \multicolumn{3}{|c|}{ Local Quadratic } \\
\hline & $\begin{array}{c}\text { AMSE } \\
(1) \\
\end{array}$ & $\begin{array}{c}\text { Variance } \\
(2)\end{array}$ & $\begin{array}{c}\text { Bias }^{2} \\
(3)\end{array}$ & $\begin{array}{c}\text { AMSE } \\
(4) \\
\end{array}$ & $\begin{array}{c}\text { Variance } \\
\text { (5) }\end{array}$ & $\begin{array}{c}\text { Bias }^{2} \\
(6) \\
\end{array}$ \\
\hline Bottom Kink & 12.51 & 12.47 & 0.04 & 2434 & 2406 & 28 \\
\hline Top Kink & 3.98 & 3.17 & 0.81 & 20.41 & 18.98 & 1.43 \\
\hline
\end{tabular}

Table entries are estimates of AMSE for the local linear and local quadratic estimators under the default CCT bandwidth, with asymptotic variance and asymptotic squared bias components. 


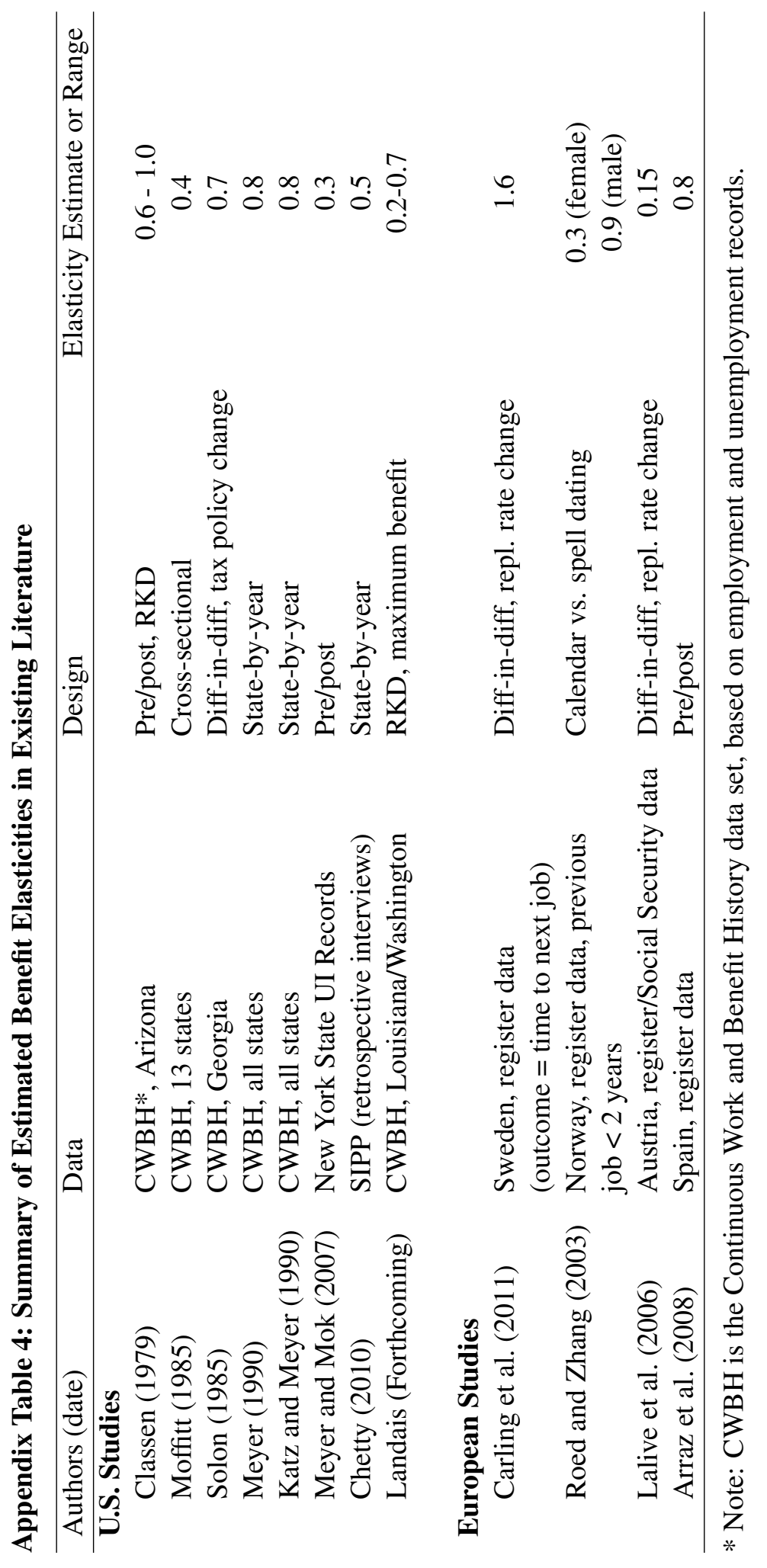

